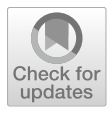

Cite as

Nano-Micro Lett.

(2021) 13:119

Received: 23 November 2020

Accepted: 22 January 2021

Published online: 3 May 2021

(C) The Author(s) 2021

\section{Bottom-Up Engineering Strategies for High-Performance Thermoelectric Materials}

\section{HIGHLIGHTS}

\author{
Qiang Zhu ${ }^{1}$, Suxi Wang ${ }^{1}$, Xizu Wang ${ }^{1}$, Ady Suwardi ${ }^{1}$, Ming Hui Chua ${ }^{1}$, \\ Xiang Yun Debbie Soo ${ }^{1}$, Jianwei $\mathrm{Xu}^{1,2} \otimes$
}

- Recent advances of various bottom-up approaches for constructing nanostructured semiconductor thermoelectric materials with different dimensions are reviewed.

- The relationships between the nanostructures and the key electronic and thermal transport parameters contributing to ZT are discussed.

- The challenges of the bottom-up strategies and suggestions for future development toward thermoelectric applications are provided.

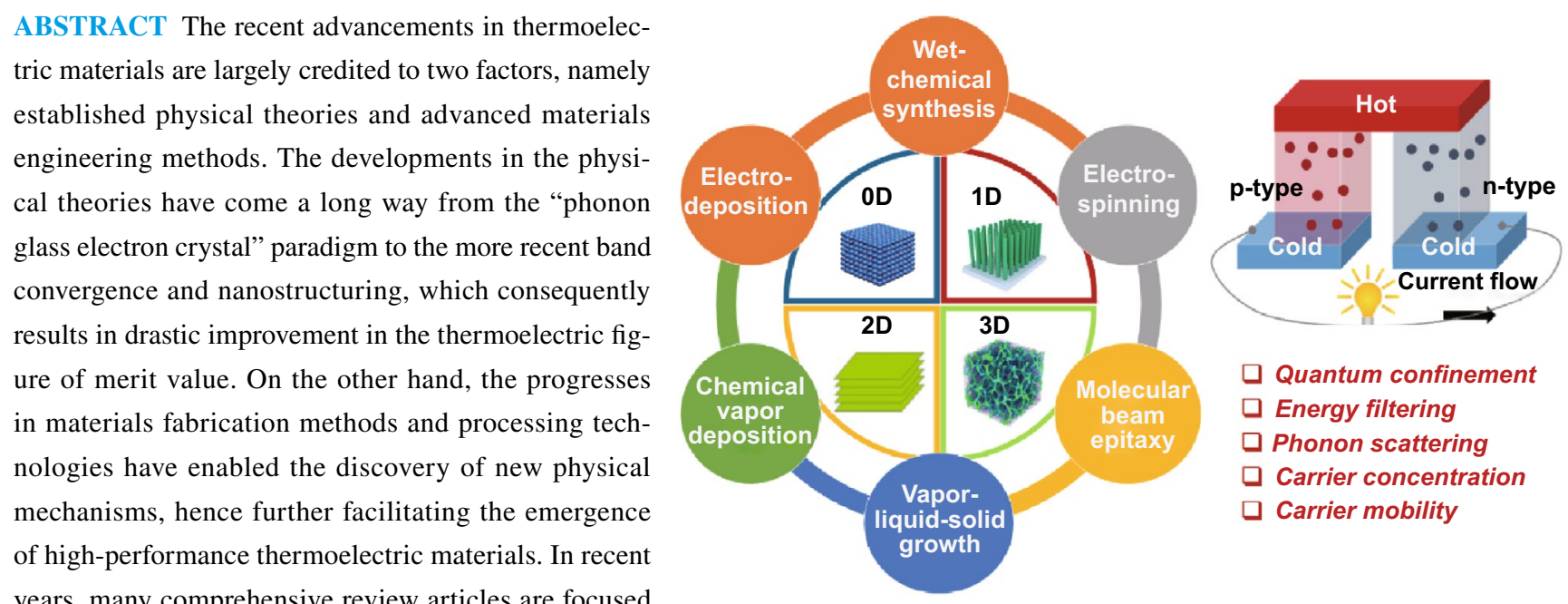
years, many comprehensive review articles are focused on various aspects of thermoelectrics ranging from thermoelectric materials, physical mechanisms and materials process techniques in particular with emphasis on solid state reactions. While bottom-up approaches to obtain thermoelectric materials have widely been employed in thermoelectrics, comprehensive reviews on summarizing such methods are still rare. In this review, we will outline a variety of bottom-up strategies for preparing high-performance thermoelectric materials. In addition, state-of-art, challenges and future opportunities in this domain will be commented.

KEYWORDS Thermoelectric; Nanostructures; Bottom-up; Synthesis; Nanomaterials

Qiang Zhu and Suxi Wang have contributed equally to this work.

$\triangle$ Jianwei Xu,jw-xu@imre.a-star.edu.sg

1 Institute of Materials Research and Engineering, A*STAR (Agency for Science, Technology and Research), 2 Fusionopolis Way, Innovis, \#08-03, Singapore 138634, Singapore

2 Department of Chemistry, National University of Singapore, 3 Science Drive 3, Singapore 117543, Singapore 


\section{Introduction}

The ever-increasing demand on electricity has driven the expansion of electricity supply sources, such as solar energy, nuclear power and photovoltaics. All these electricity sources are alternatives to the conventional fossil fuels. However, all these power generation approaches cannot address more than $60 \%$ of energy loss worldwide as waste heat. The ubiquity of low-grade waste heat $\left(<200{ }^{\circ} \mathrm{C}\right)$ in modern electronic devices is an opportunity in terms of energy recovery. In a bid to utilize the waste heat, thermoelectrics becomes is a viable option as it converts a thermal gradient into electrical energy in the solid state. This type of conversion from thermal heat to electricity possesses a lot of advantages, such as silent mode, no noise and less pollution, which has also triggered the development of various niche applications, including radioisotope thermoelectric (TE) generators (TEGs) for the space exploration, for example, National Aeronautics and Space Administration's Voyager 1 and 2. However, all these applications put forward strict requirements for energy conversion efficiency, and traditionally, high-performance inorganic semiconducting materials, such as bulk $\mathrm{Bi}_{2} \mathrm{Te}_{3}$, $\mathrm{SnSe}$, and PbTe [1-6], have been extensively studied and fabricated into the TEGs.

The efficiency of TE materials can be expressed by dimensionless figure of merit $\left(Z T=\sigma S^{2} T / \kappa\right)$, where $S, \sigma, T$, and $\kappa$ represent Seebeck coefficient, electrical conductivity, absolute temperature, and thermal conductivity, respectively. In order to enhance $Z T$, a large $S$, a high $\sigma$, and a lower $\kappa$ are preferred, but these parameters are inter-dependent on each other. For instance, while the $S$ has an inverse dependency on the carrier concentration $(n)$, the $\sigma$ is proportional to the $n$. To optimize the power factor $\left(P F=\sigma S^{2}\right)$ and $Z T$, the $n$ needs to be optimized either via doping or defect engineering to be around $10^{19} \mathrm{~cm}^{-3}$ level $[7,8]$. However, the introduced dopants can deteriorate the charge carrier mobility, thus hindering the magnitude of the optimal power factor [9, 10]. A popular and effective strategy for enhancing the $P F$ is via the electronic band-engineering [11-16]. In the electronic band-engineering, either the band-convergence or the effective mass manipulation can be explored to increase the $S$ or $\sigma$, respectively $[13,17,18]$. In addition to the $P F$, the $\kappa$ is also an important parameter that affects the $Z T$ value. The $\kappa$ is composed of electrical thermal conductivity $\left(\kappa_{\mathrm{e}}\right)$ and lattice thermal conductivity $\left(\kappa_{\mathrm{L}}\right)$. The $\kappa_{\mathrm{e}}$ value is predominantly affected by the $\sigma$ through the charge carrier concentration and the $\kappa_{\mathrm{L}}$ is controlled by the introduced impurities through various phonon scattering mechanisms. The close inter-correlation between the $S, \sigma$, and $\kappa$ makes it highly challenging to achieve a high $Z T$ value.

Another useful approach to improve $Z T$ is to prepare nanostructured semiconducting materials. The advantages of nanostructuring TE materials offer a pathway to positively decouple the correlation between the $S, \sigma$, and $\kappa$. The quantum confinement effects associating with these nanostructures help to alter the electronic density-of-states, and therefore improve the $P F$ [19]. In addition, another noteworthy feature of nanostructuring is that it is able to decrease the $\kappa_{\mathrm{L}}$ through respective phonon scattering effects. For instance, one approach to reduce the $\kappa_{\mathrm{L}}$ is to minimize the phonon relaxation time through the introduction of phonon scattering sources such as point defects, dislocations and interfaces [20].

Traditionally, a popular method to prepare nanostructured TE materials is to make use of a top-down approach, including ball milling, exfoliation, annealing, and so on. However, most of these processes are energy-consuming. Additionally, the precise control of the particle size, size-distribution and shape remains challenging. In order to address these issues, bottom-up approaches is sought after. The primary advantage of bottom-up approach lies in its versatility in designing nanostructured materials, which is favorable for phonon scattering (i.e., lower thermal conductivity). Approaches such as liquid-phase synthesis, vapor-liquid-solid (VLS) growth, solution-liquid-solid (SLS) growth, chemical vapor deposition (CVD), electrochemical deposition, electrospinning, and so on have provided a wide variety of nanoscaled architectures, offering many advantages over some traditional methods in controlling phase purity, crystallinity, density and dimensions of nanostructures. For nanomaterial-based thermoelectric devices, the nanoscaled particles can be assembled at a macroscopic scale and homogeneously distributed, either in thin film or in bulk materials. The final thermoelectric properties will very much depend on not only the individual nanoparticles, but also the manner they assemble. For instance, by controlling annealing temperature and/or pressure, various nano-sized defects can be engineered, as shown in Fig. 1. Consequently, by engineering multiscale defects such as point defect (nanoparticle), nano-precipitates, and line dislocations, it is possible to scatter phonons of a wide-range of wavelengths, resulting in very low $\kappa_{\mathrm{L}}$. Another 


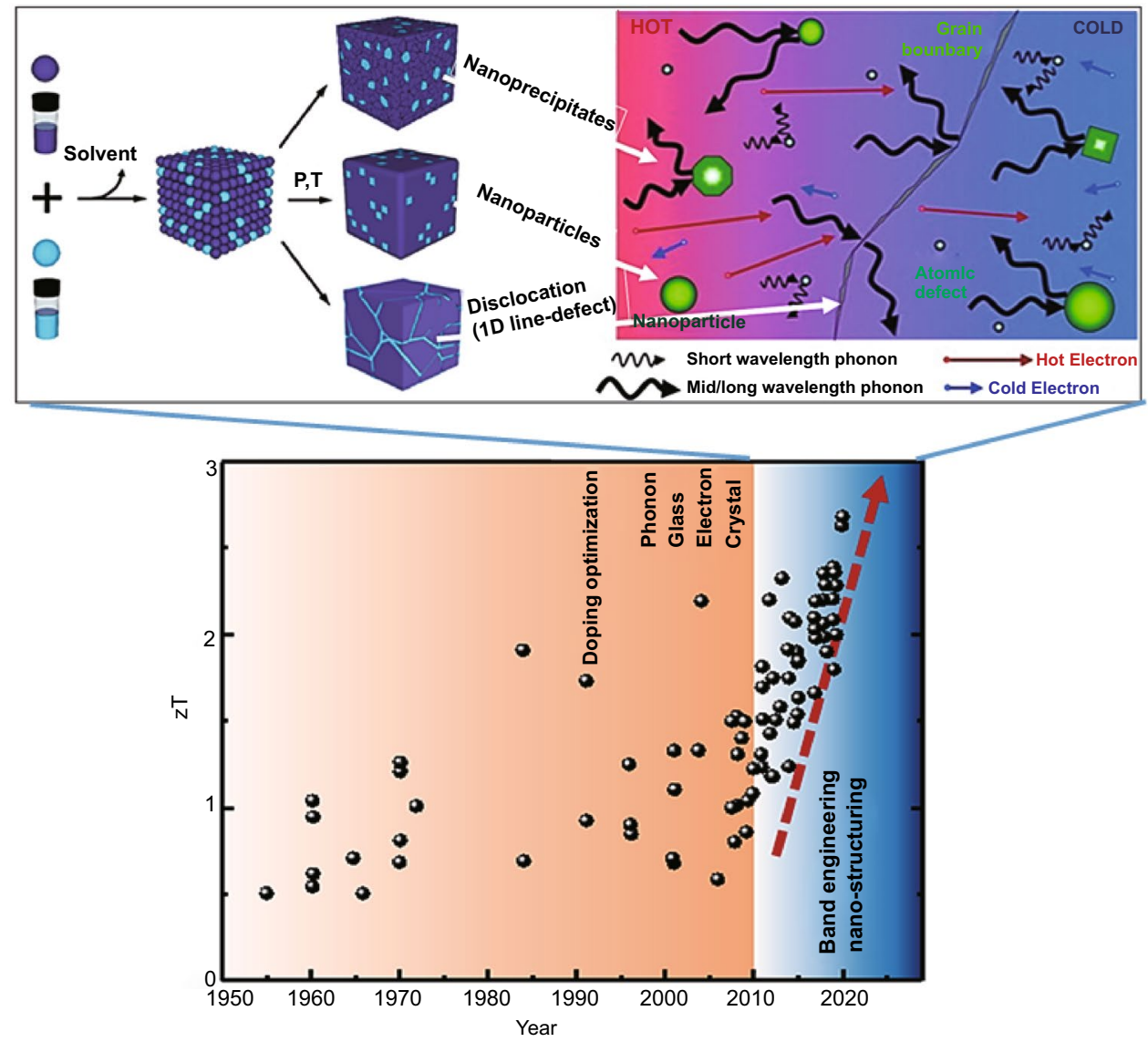

Fig. 1 Illustrations showing the bottom-up assembly to synthesize nanoscaled thermoelectric materials and the advantageous effects brought about by multi-wavelength phonon scattering due to the various shape, and size defects brought about by these bottom-up approaches. Adapted with permission from Ref. [22, 27]

advantage of bottom-up approaches is that they generally do not require a substrate to support the growth, therefore enabling higher throughput than traditional methods such as physical vapor deposition. This paper will cover the recent studies of using various nanostructures to tune the TE performance through bottom-up engineering strategies, which have, however, not extensively been attracted attention in existing reviews on the nanostructured TE materials [8, 21-26].

\section{Benefits of Nanostructuring}

Understanding the inter-correlation between different TE parameters that make up the $Z T$ can be a useful guide to design nanostructured TE materials. Through extensive studies on the electron and phonon transport and electron-phonon couplings, multiple strategies have been investigated and applied for the enhancement of the TE performance.
In this section, these strategies including size effect, mean free path and quantum effect, energy filtering effect at grain boundary, phonon scattering effect by nanoparticles as well as other related effects, will be summarized in details, embodying the latest understanding and effective manipulation of the interplay among carrier, phonon, lattice, interface and electronic states in TE nanomaterials.

\subsection{Nanostructuring Effect in Thermoelectrics}

Conceptually, in order to obtain a high $Z T$ value, both the $S$ and $\sigma$ must be large, while the $\kappa$ must be minimized so as to maximize the power output at a high temperature difference. Traditionally, there are two main design principles in searching for bulk TE materials with high $Z T$ values. The first approach is the "phonon glass electron crystal" approach, and the other approach is the nanostructuring of TE materials. To achieve 
the phonon glass electron crystal, materials with complex structures are in general preferred [11, 28-31]. Recently, TE materials with exceptionally high $Z T$ values of above 2 have been reported owing to the advance of various materials development approaches, including band engineering, defect optimization and nanostructuring. Nanostructuring is a promising way to improve the TE performance by means of reduction of the characteristic length of the phonons mean-free paths [8, 32-34]. It is generally accepted that the mean-free paths for phonons are much longer than that of electrons, and therefore by judiciously tuning the nanostructure size to the same order as the phonon mean-free paths, it is possible to selectively scatter phonons and not electrons, resulting in a lower $\kappa_{\mathrm{L}}$ while maintaining a high $\sigma[35,36]$.

The classical nanostructuring effects concern the scattering-limited mean free paths and the confinement-induced variation in the electronic dispersion relation, respectively. For instance, heat is carried by phonons with a wide, momentum and energy temperature-dependent spectrum, prohibiting the $k_{\mathrm{L}}$ by limiting the phonon mean-free path over a broad temperature range therefore requiring all-scale hierarchical nanostructuring and microstructuring [37]. Under the circumstance of the classical size effect, the lowest possible $k_{\mathrm{L}}$ is defined as the amorphous limit, when the phonon mean-free path gets as small as the interatomic spacing and heat is carried by the random-walking Einstein mode, just like in amorphous materials. The minimum lattice thermal conductivity $k_{\min }$ can be expressed as [38]:

$\kappa_{\min }=\left(\frac{\pi}{6}\right)^{\frac{1}{3}} k_{B} n_{a}^{\frac{2}{3}} \sum_{i} v_{i}\left(\frac{T}{\theta_{i}}\right)^{2} \int_{0}^{\theta_{i} / T} \frac{x^{3} e^{x}}{\left(e^{x}-1\right)^{2}}$

where the $\theta_{i}=v_{i}\left(\hbar / k_{B}\right)\left(6 \pi^{2} n_{a}\right)^{1 / 3}$ is the cutoff frequency, $n_{a}$ is the number of atoms per unit volume, $\hbar$ is the Planck's constant $h$ divided by $2 \pi$, and $\nu_{i}$ is the sound velocity vector.

Theoretical and experimental reports show that the high density of nanoscaled grains can induce the effective phonon scattering of over $60 \%$ and remarkably reduce the $\kappa$ [39]. In addition, due to the low dimension of nanostructures, the phonon confinement effects must be considered. For instance, the Umklapp scattering process in superlattice is different from those of bulk materials. The so-called miniUmklapp arises due to the periodically alternating layers of a material that has a large superlattice constant, resulting in a mini-Brillouin zone, and hence a lower $\kappa$ [40].

\subsection{Quantum Effect}

Low dimensional materials/nanostructures are defined by a characteristic dimension in order comparable to the de Broglie wavelength of charge carriers. As a result, the degree of freedom of the carrier motion is restricted by the dimension of the nanostructures. Consequently, the electronic transport behavior is drastically altered, resulting in the so-called quantum size effect [41]. Figure 2 illustrates the electronic band structure behavior of various low dimensional materials as compared with traditional three-dimensional (3D) bulk materials. Compared with 3D materials, all low dimensional materials have a very sharp feature in the density of states, especially for one-dimensional (1D) and zero-dimensional (0D) materials. These features are very beneficial to enhance the $S$ and thus the $P F[42,43]$. Therefore, this quantum size effect underlies the paradigm of TE nanostructuring [44-46].

To understand the origin of the $S$ enhancement in low dimensional materials, it is helpful to look into the Mott equation shown below:

$S=\frac{\pi^{2} \kappa_{B}^{2} T}{3 e}\left[\frac{1}{n} \frac{\partial n(E)}{\partial E}+\frac{1}{\mu} \frac{\partial \mu(E)}{\partial E}\right]_{E=E_{F}}$

The first and second terms inside the square bracket represent the contribution to the $S$ due to the carrier concentration modulation and carrier mobility modulation, respectively. In

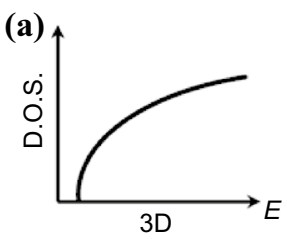

Bulk Semiconductor

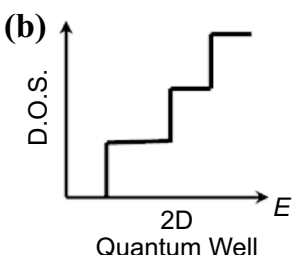

Quantum Well
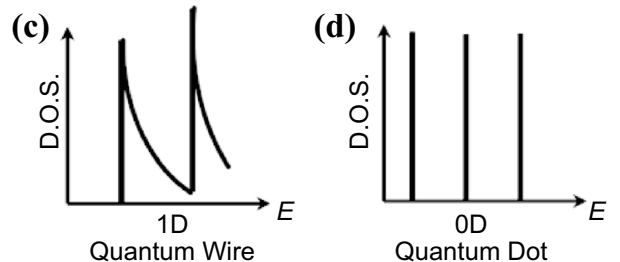

Fig. 2 Illustrations showing the electronic density-of-states of bulk and low dimensional materials. a Bulk semiconductor. b 2D materials. c 1-D materials (i.e., nanowires (NWs), nanorods). d Quantum dots. Adapted with permission from Ref. [47] 
most TE studies, the second term (carrier mobility modulation) is ignored because of the low energy dependence of mobility (i.e., $r=-0.5$ for the acoustic phonon scattering). The density of states $v s$ the energy profile shown in Fig. 2 is directly proportional to the first term in Eq. 2, $\partial n(E) / \partial E$. Therefore, a higher slope in the density of states $v s$ the energy will result in a higher $S$. Theoretically, the enhancement in the $S$ in low dimensional materials has been predicted in several literatures for 2D superlattice as well as 1D NWs [48-52]. More recently, numerous experimental enhancements on the $S$ in low dimensional materials have been reported, such as $\mathrm{Pb}_{1-x} \mathrm{Eu}_{x} \mathrm{Te} / \mathrm{PbTe}$ multiple quantum wells and $\mathrm{PbTe}_{1-x} \mathrm{Se}_{x} / \mathrm{PbTe}$ quantum dot superlattice [53, 54].

In addition to low dimensional materials, the concept of enhancing the slope of density of states $v s$ the energy has been successfully applied using resonant doping in bulk 3D materials. Most notably, elements $\mathrm{Tl}$ and In have been shown to be a resonant dopant for $\mathrm{PbTe}$ and GeTe, respectively [12, $55,56]$. These observation suggests that physical intuition derived from the studies of low dimensional materials can also be applied to a bulk materials system.

\subsection{Energy Filtering Effect}

The first concept of energy filtering was introduced by Ioffe in 1959 [57] and further investigated by Rowe and Min in 1995 [58]. They studied the effect of different barriers on the $\sigma$ and the $S$ by the relaxation-time approximation method, indicating that the flow of minority charge carriers should become obstructed by the high-energy barriers, thereby suppressing the bipolar effects. This presented obvious reduction of the $\sigma$ by promoting transport of the primary charge carriers. Nanostructures can also be used to enhance the $P F$ via the energy filtering. The presence of nano-sized precipitates can act as a filter for carriers with low energy, therefore increasing the $S$ and hence the $P F$. An illustration of the energy filtering mechanism is shown in Fig. 3. It is noteworthy that the overall benefit of the energy filtering is debatable, even to date. On one hand, by filtering out the low energy carriers, the $S$ can be improved. On the other hand, the fewer number in carriers leads to a decrease in the $\sigma$. More recently, the manifestation of the energy filtering was elucidated in terms of the scattering exponent $(r)$. The change in $r$ from -0.5 for the acoustic phonon scattering

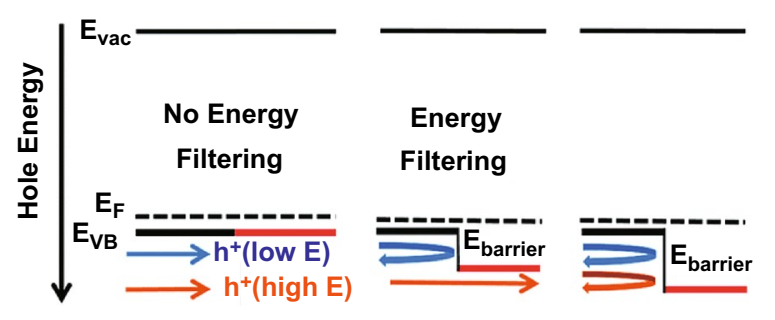

Fig. 3 An illustration of the energy filtering effect. In the middle image, carriers (holes) with high energy (red-arrow) travel unobstructed through the energy barrier while carriers with lower energy (blue) are blocked. Adapted with permission from Ref. [60]. (Color figure online)

to near 0 due to the energy filtering has been shown to be greatly beneficial for enhancement in TE performance [59].

\subsection{Phonon Scattering Effect by Nanoparticles}

In low dimensional or nanostructured TE materials, in addition to the phonon-phonon scattering as the dominant effect, the phonon scattering effect due to nanostructures cannot be negligible. Recently, it is generally accepted that the nanoparticles or nanocrystals can be easily in-situ in bulk materials to obtain the lower $\kappa$ [8]. Here, the phonon relaxation time of nanoparticle scattering $\left(\tau_{N P}\right)$ is given by the Mathiessen-type interpolation between the short- and long-wavelength scattering regimes [61].

$$
\begin{aligned}
& \frac{1}{\tau_{N P}}=\nu\left(\sigma_{S}^{-1}+\sigma_{1}^{-1}\right)^{-1} \\
& \sigma_{S}=2 \pi R^{2} \\
& \sigma_{1}=\pi R^{2} \frac{4}{9}\left(\frac{\Delta D}{D}\right)^{2}\left(\frac{R}{\nu}\right)^{4}
\end{aligned}
$$

The parameter $\rho$ is the density of a nanoparticle/nanocrystal, $R$ is the radius of a nanoparticle/nanocrystal, $D$ is the density of the matrix, and $\Delta D$ is the difference in densities between a nanoparticle/nanocrystal and matrix. Kim et al. observed an apparent reduction in the $\kappa$ by almost a factor of 2 below the limit of alloy when embedding the ErAs nanoparticles into $\mathrm{In}_{0.53} \mathrm{Ga}_{0.47} \mathrm{As}$ [62]. According to the simulation of the $\kappa$ of $\mathrm{Si}-\mathrm{Ge}$ nanocomposite [63], SiGe nanoparticle-in-alloys [61], an observable decrease in the $\kappa$ was reported. In previous studies, Kundu et al. noted that the decrease in the $\kappa$ of nanoparticles materials of matrix 
depended on the relative atomic mass difference between the nanoparticle and matrix, which is consistent with Eq. (3) $[64,65]$. Similar reduction of the $\kappa$ was also observed in various TE materials with nanostructuring such as $\mathrm{PbTe}[66$, 67], PbS [68, 69], and SnTe [70], thus improving the ultimate TE performance.

\section{Bottom-up Nanostructuring of Thermoelectrics}

To date, the most popular method to prepare thermoelectrics is to use conventional solid-state sintering that involves ball milling and/or spark plasma sintering (SPS), which is energy-consuming and lack of mechanisms to precisely control the size, shape and surface chemistry. In comparison with the top-down nanostructuring or nanopatterning such as electron beam lithography [71], the bottom-up approaches are relatively cost-effective and also offer advantages in controlling phase purity, crystallinity, density and dimensions. Nanostructured or nanocomposite thermoelectric materials can help enhance $Z T$ via increasing power factor through modulation doping, decreasing thermal conductivity via phonon scattering [72], increasing $S$ by modulating the density of states of carriers, and energy filtering which results in simultaneous increase in $S$ and $\sigma$ [73]. In addition, nanomaterials may help to enhance mechanical properties via precipitation hardening which pins dislocations from moving [74]. However, the difficulties in designing nanostructured thermoelectrics lie in strong inter-correlation between materials transport properties, which demand careful adjustment of carrier densities. Therefore, tuning the size, shape, composition and phase is important in addition to distribution and orientation of these nanostructures. This is to ensure the coherence and band alignment between different phases in nanostructured TE materials, and modulate the effect of defects (dislocations, point defects, and stacking faults) and surface roughness on the thermoelectric properties such as thermal conductivity.

In terms of materials processing, vacuum-based thin film deposition methods are the only mature technologies to date that can reliably produce nanostructured materials and accurately control the composition or stoichiometry. In thin film thermoelectrics, some of the highest ever reported power factor have been reported [75]. Nevertheless, the main drawback from such vacuum deposition is its high cost and low productivity. In addition, it cannot be so easily scaled up to obtain bulk or thick films, and even not to mention the various issues in accurately measuring the thermoelectric properties, especially $S$ and $\kappa$. During these measurements, the heat from hot side not only transfers to cold side, but also to the substrate underneath the film, which makes data interpretation extremely challenging. In comparison, nanostructuring via solution-based processes is very attractive because it does not require substrate (often made of expensive single crystal) to support the growth and can be readily scaled up. The solution-based bottom-up processes also provide convenience for the device fabrication in terms of size, shape, flexibility and conformability, which is promising for application in wearable thermoelectric energy harvest systems.

In this section, the recent progress on various bottomup approaches towards the preparation of nanostructured thermoelectric materials with different dimensions will be summarized, their structure-property relationships as well as mechanisms for performance enhancement will also be discussed.

\subsection{D Nanoparticles and Nanoinclusion}

\subsubsection{Colloidal Synthesis}

At present, vapor-phase approaches are generally found not suitable for the synthesis of high-quality nanocrystals due to existing limitations found in instruments and precursors [76-78]. In contrast, the liquid-phase colloidal synthesis of monodisperse semiconductor nanocrystals can offer a convenient route towards low-cost and scalable low-dimensional TE materials. In addition, the optoelectronic properties of nanocrystals can be tuned via synthesis, engineering surface of nanocrystals and control of the size down to sub-10 nm range. This opens up the possibility to explore properties of TE materials with strong quantum-confine effect [79]. The use of capping ligands and surfactants facilitates the dispersion of colloidal semiconductor nanocrystals in solvents. The shapes and sizes of nanocrystals can be easily tailored by making use of the kinetic control over the nucleation and growth processes with the assistance of organic ligands. The colloidal semiconductor nanocrystals exhibit attractive TE features owing to the low dimensionality of the materials. On one hand, abundant grain boundaries are able to scatter the phonons to reduce the $\kappa$. On the other hand, the quantum 
confinement effect brings about increased density of states near the Fermi level, giving rise to an enhanced $S$. Moreover, the stable colloidal suspensions are highly solutionprocessable, making them particularly attractive for ultrahigh throughput device manufacturing such as spin-coating, inkjet printing and roll-to-roll casting $[80,81]$.

Despite the above advantages, the major obstacle of exploiting solution-processed nanocrystals for high-performance TE devices is that organic ligands are insulating in nature, hindering charge transfer between nanocrystals. Furthermore, the large interface of nanocrystals adversely affects the charge transfer processes, and thus leads the $\sigma$ of nanocrystal films to inevitably low. Therefore, the key to improve the TE performance of nanocrystals is to find an effective way to remove the organic ligands on the surface of the nanocrystals after synthesis, and properly engineer electronic coupling at the interface of nanocrystals, while maintaining the nanocrystal features such as the quantum confinement effect and interfaces.

In 2008, Wang et al. prepared colloidal PbSe nanocrystals with sizes from $\sim 4.3$ to $\sim 8.6 \mathrm{~nm}$ by reacting lead oleate with tri-n-octylphosphine selenide in squalane in the presence of oleic acid capping ligands [82]. Oleic acid at the nanocrystal surface was then stripped by hydrazine, which reduced the interparticle spacing from $\sim 1.1$ down to $0.4 \mathrm{~nm}$ and resulted in a greatly improved $\sigma$. In 2013, Yang et al. synthesized PbSe colloidal quantum dots (CQD) with different sizes using conductive metal chalcogenide complexes $\mathrm{SnS}_{2}-\mathrm{N}_{2} \mathrm{H}_{4}$ to replace the organic ligands (Fig. 4a) [83]. It was found that the films of smaller QDs have a larger $S$, indicating the presence of stronger quantum confinement, and lower $\sigma$ and $\kappa$ values. The prepared $\operatorname{PbSe}\left(\mathrm{SnS}_{2}\right)$ QD films displayed enhanced $Z T$ from 0.5 at room temperature to $1.0-1.37$ at $400 \mathrm{~K}$ (Fig. 4b). In 2016, Ding et al. reported a convenient approach to fabricate spin-coated thin films with colloidal lead chalcogenide nanocrystals using a twostep interface engineering treatment (Fig. 4e): (1) the ligand exchange process was performed on the $\mathrm{PbTe}$ or $\mathrm{PbTe} / \mathrm{PbS}$ layer by treatment with ethylenediamine, and (2) the posttreated layer was then annealed at different temperatures for proper coupling of the nanocrystals [84]. Notably, the nanocrystal thin films were found to exhibit have a higher $S$ (400-460 $\mu \mathrm{V} \mathrm{K}^{-1}$ ) than that of bulk PbTe, which is believed to be ascribed to the effect of the quantum confinement of
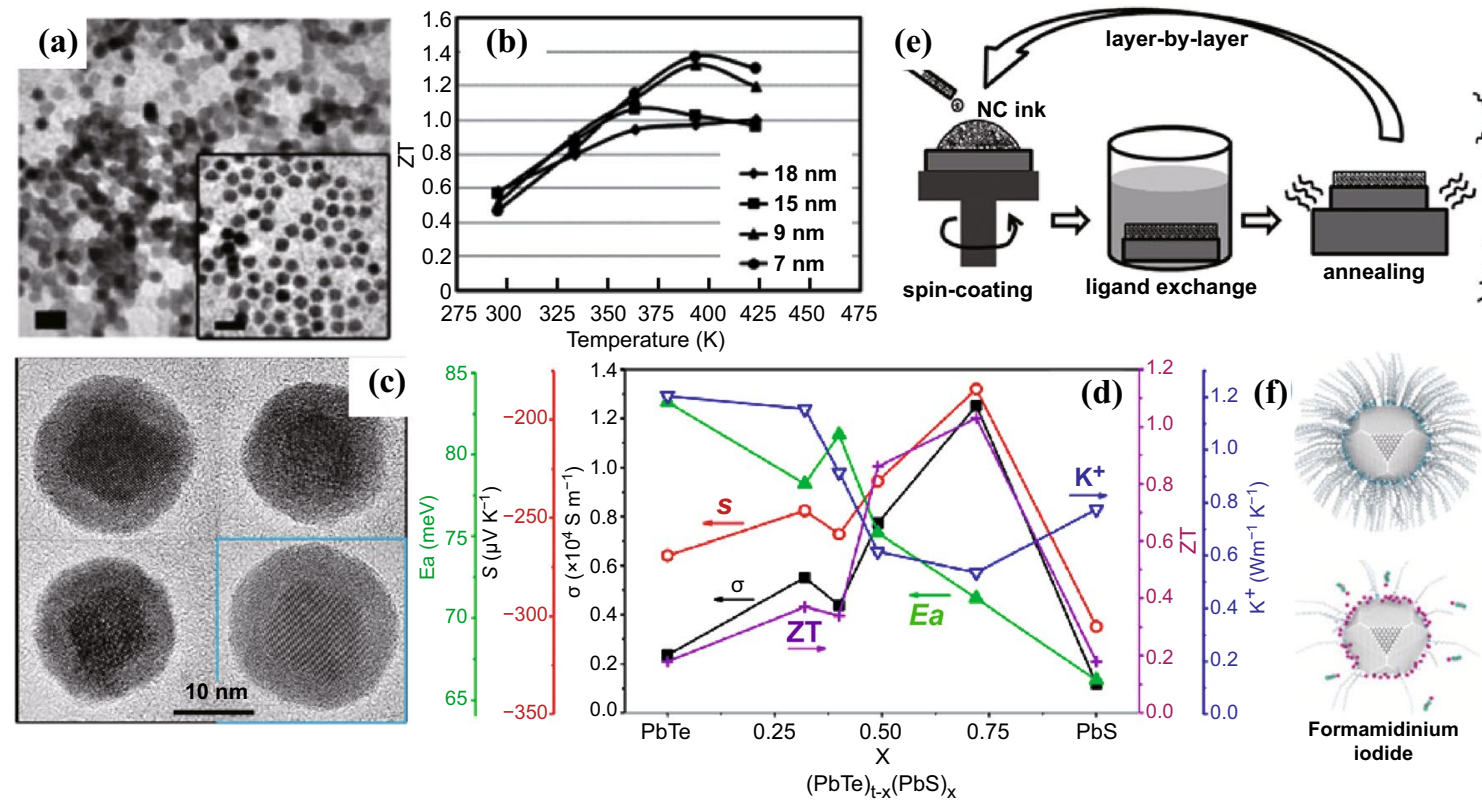
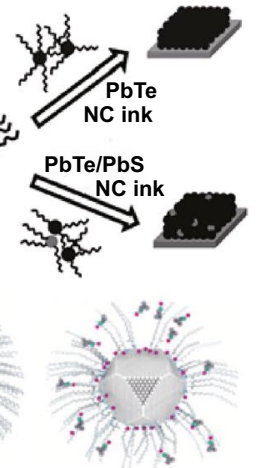

Isobutylammonium

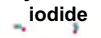

$-$

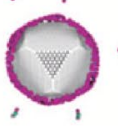

Methylammonium iodide

Fig. 4 a TEM image of colloidal PbSe QDs after metal chalcogenide complex treatment with a diameter of $9 \mathrm{~nm}$. b $Z T$ of the prepared $\mathrm{PbSe}\left(\mathrm{SnS}_{2}\right)$ QD films versus temperature. c HRTEM graphs of a few $(\mathrm{PbTe})_{0.25} @(\mathrm{PbS})_{0.75}$ core-shell nanoparticles. d $\sigma, S$, porosity-corrected $\kappa$, and $Z T$ at $710 \mathrm{~K}$ and activation energy for electrical transport in the low-temperature range $\left(\mathrm{E}_{\mathrm{a}}\right)$, as a function of the $\mathrm{PbS}$ concentration in the core-shell $(\mathrm{PbTe})_{1-x}(\mathrm{PbS})_{x}$ nanoparticles. e Synthesis of colloidal nanocrystal thin films by layer-by-layer method and strategy for optimizing TE performance. f Schematic of oleic acid ligand exchange using different iodide salts. Adapted with permission from Refs. [83-86] 
the nanocrystals. The $S$ of all the PbTe nanocrystal thin films was observed to decrease with increasing annealing temperature, likely due to the weakened quantum confinement effect of the fused nanocrystals. It was also found that ligand replacement with ethylenediamine could assist in necking between nanocrystals, resulting in an increased $\sigma$. The fabricated $\mathrm{PbTe} / \mathrm{PbS}$ nanocrystal thin films exhibited a high $\mathrm{ZT}$ value of $\approx 0.30$ at $405 \mathrm{~K}$ after thermal annealing at $400{ }^{\circ} \mathrm{C}$.

The replacement of short-chain organic ligands to modify colloidal nanocrystals leads to strong inter-particle electronic coupling and thus promotes efficient charge transport in colloidal nanocrystals films. In addition to offering excellent surface defect passivation, the use of appropriate halides in engineering the electronic coupling in nanocrystal films would be also crucial for efficient charge transport. In 2019, Nugraha et al. reported n-type TE iodide-capped PbS CQD film which allows for the fabrication of highly efficient TEG devices [85]. The counter-ions in iodide salts were found to play a critical role in facilitating ligand removal and charge transport in CQD films (Fig. 4f). Methylammonium iodide (MAI) could bring about efficient charge transport in the QD films which was resulted from the complete removal of oleic acid ligands and excellent passivation of surface defects. An impressive improvement in the $\sigma$ of $100 \%$, exceeding $12 \mathrm{~S} \mathrm{~cm}^{-1}$, was obtained for the MAI-treated CQD films, leading to a promising n-type $P F$ of up to $24 \mathrm{Mw} \mathrm{m}^{-1} \mathrm{~K}^{-2}$ at relatively low temperatures $(<360 \mathrm{~K})$, which was significantly improved compared to previously reported n-type lead chalcogenide CQD films $\left(<1 \mu \mathrm{W} \mathrm{m} \mathrm{m}^{-1} \mathrm{~K}^{-2}\right)$.

In 2013, Ibanez et al.reported a one-pot two-step colloidal synthetic route to prepare PbTe@PbS core-shell structured nanoparticles with narrow size distributions and exceptional composition control (Fig. 4c, d) [86]. As-synthesized PbTe@ $\mathrm{PbS}$ nanoparticles served as the building blocks for bottomup production of $\mathrm{PbTe}-\mathrm{PbS}$ nanocomposites. Interestingly, a doping-like effect was observed when $\mathrm{PbTe}$ and $\mathrm{PbS}$ were mixed at the nanometer scale. In such PbTe-PbS nanocomposites, synergistic nanocrystal doping effects resulted in up to tenfold increase in the $\sigma$ compared to pure $\mathrm{PbTe}$ and $\mathrm{PbS}$ nanomaterials alone. Without intentionally doping of any of the two phases, $(\mathrm{PbTe})_{0.28}(\mathrm{PbS})_{0.72}$ reached $\sigma$ up to $1.2 \times 10^{4}$ $\mathrm{S} \mathrm{m}^{-1}$. At the same time, the acoustic impedance mismatch between $\mathrm{PbTe}$ and $\mathrm{PbS}$ phases and a partial phase alloying collectively provided $\mathrm{PbTe}-\mathrm{PbS}$ nanocomposites with a significantly reduced $\kappa$ (down to $0.53 \mathrm{~W} \mathrm{~m}^{-1} \mathrm{k}^{-1}$ ). As a result, a high TE ZT of $\sim 1.1$ was obtained at $710 \mathrm{~K}$.

\subsubsection{Hydrothermal/Solvothermal Synthesis}

Hydrothermal synthesis involves the growth of crystals with different sizes at the submicron to nanometer scale. This is usually achieved via chemical reactions in an aqueous medium, at elevated temperature and high pressure. The successful synthesis of the nanostructures is highly dependent on the precise control over the internal reaction conditions, such as reaction time, pressure, $\mathrm{pH}$ value, reagent concentration, and presence of organic additives or templates, as well as external reaction environment including microwave or conventional heating. The solvothermal method can be employed to have more control over the size, shape, reactivity, and phase of the nanostructures in organic solvents than in water. The viscosity and polarity of solvent can influence the transport behavior and solubility of the reagents in the liquid medium, and hence the properties of nanostructured product. Although there are many reports on the preparation of semiconductor nanocrystals using hydrothermal/ solvothermal methods [87-90], these approaches generally require high temperature and pressure as well as prolonged reaction periods, which significantly hinder applications for large-scale synthesis.

In 2016, Li et al. reported a facile, rapid, environmentallygreen and high-yield microwave hydrothermal method for preparing SnTe nanoparticles with controlled sizes from micro-scale to nano-scale [91]. The reaction rates can be controlled by adjusting the concentration of reagents, resulting in SnTe nanoparticles with sizes ranging from 165 to $8.2 \mathrm{~nm}$. After the SPS treatment, an ultra-low $\kappa$ of $0.60 \mathrm{~W} \mathrm{~m}^{-1} \mathrm{~K}^{-1}$ at $800 \mathrm{~K}$, being only $11.8 \%$ of the reference sample, was obtained using the $165 \mathrm{~nm}$ nanoparticles (Fig. 5a) owing to the enhanced phonon scattering effect introduced by refined grains, grain boundaries and point defects in the sintered dense materials. This sintered materials (Fig. 5b) exhibited a relatively higher $S\left(58-90 \mu \mathrm{V} \mathrm{K}^{-1}\right.$, $323-800 \mathrm{~K}$ ) and much a higher $Z T$ value (about 0.49 at $803 \mathrm{~K}$ ) compared to pure SnTe bulk material, which can be ascribed to the enhanced phonon scattering and the intensified energy filtering effect. The size effect was also found to have some influences on the $\sigma$. The electronic transport mechanism (Fig. 5c) was proposed and the hole mobility, carrier concentration and the effective mass $\left(m_{x} * / m_{0} *\right)$ as function of the decreased grain sizes were measured by the corresponding carrier mobility test as shown in Fig. 5d. Evidently, the hole mobility reduced from 125 to $31 \mathrm{~cm}^{2} \mathrm{~V}^{-1} \mathrm{~s}^{-1}$ 

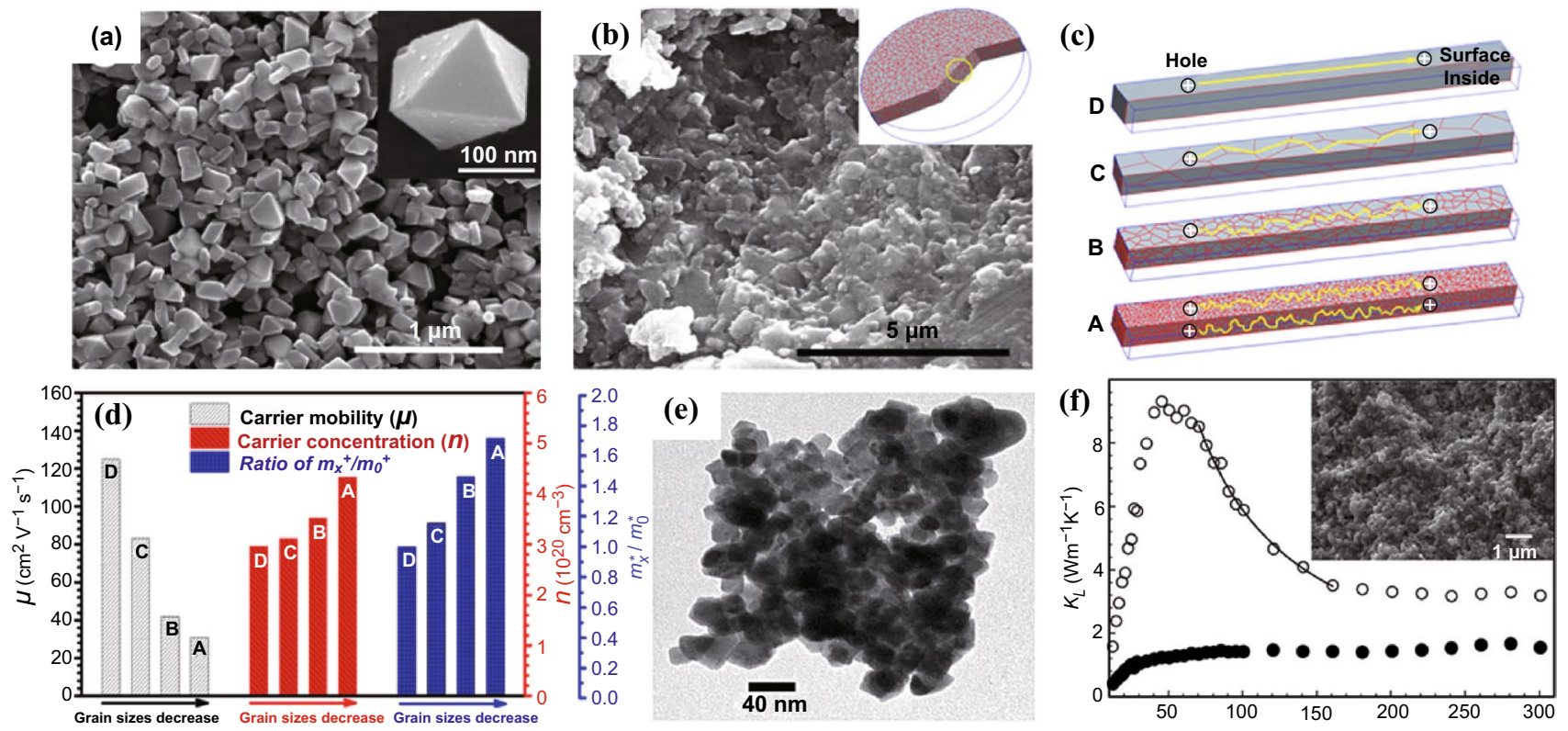

Fig. 5 a SEM image of the SnTe nanoparticles synthesized by microwave hydrothermal method. b SEM image of the dense specimen from the $165 \mathrm{~nm}$ nanoparticles. c Proposed electrical transporting mechanism of the dense samples sintered from the $165 \mathrm{~nm}, 550 \mathrm{~nm}$ nanoparticles (NPs), $8.2 \mu \mathrm{m}$ microparticles (MPs), and mechanically alloyed (MA) powder. $\mathbf{d}$ Hole mobility, carrier concentration and the ratio of $m_{x} * / \mathrm{m}_{0} *$ as function of the decreased grain sizes. e TEM image of $40 \mathrm{~nm} \mathrm{FeSb} b_{2}$ nanocrystals prepared by solvothermal method. f $\kappa_{\mathrm{L}}$ of the densified FeSb nanocrystals (solid circle) and bulk $\mathrm{FeSb}_{2}$ (open circle). The line of 1/T dependence (solid line) is indicative of phonon-phonon scattering. The inset shows an SEM image of a fractured surface of the densified $\mathrm{FeSb}_{2}$ specimen. Adapted with permission from Refs. [91-93]

while the carrier concentration increased when the grain sizes gradually decreased to $165 \mathrm{~nm}$ with the increasing crystal defects. It is noteworthy that the decrease in mobility is higher than the increase in carrier concentration, which results in a lower $\sigma$. In 2017, Tang et al. reported the synthesis of tetradecahedron $\mathrm{Cu}_{2} \mathrm{~S}$ microcrystals with the size of 1-7 $\mu \mathrm{m}$ via the hydrothermal method, which could achieve a $Z T$ value of 0.38 at $573 \mathrm{~K}$ after the SPS process [92]. Datta et al. also developed phase-pure $\mathrm{FeSb}_{2}$ nanocrystals with an average size of $40 \mathrm{~nm}$ (Fig. 5e) through an ethanol-mediated, low-temperature solvothermal process [93]. Without a template or capping chemical used in the process, these nanocrystals grew in their inherent orthorhombic symmetry. Due to significant grain boundary phonon scattering, the densified $\mathrm{FeSb}_{2}$ nanocomposite showed a drastic reduction in $\kappa_{\mathrm{L}}$ compared to the bulk material prepared by a solid-state synthesis process (Fig. $5 f$ ).

\subsubsection{Electrodeposition}

In addition to the above described methods, electrodeposition is another unique process known to make nanoparticles with a controlled size and morphology [94, 95], offering the benefits of fast speed, simplicity, low-cost and avoidance of use of binders. Moreover, the electrochemical deposition of TE materials enables the easy fabrication of thin TE film although this method still suffers from drawbacks, such as the existence of impurity in as-deposited thin films and poor crystallization. Therefore, it is imperative to precisely control the composition and crystallographic structure of nanoparticles to achieve an effective electrodeposition process.

In 2016, Na et al. reported a method for preparing highly conductive n-type $\mathrm{Bi}_{2} \mathrm{Te}_{3}$ nanocrystal films on a flexible substrate using electrodeposition [96]. The growth of the $\mathrm{Bi}_{2} \mathrm{Te}_{3}$ crystals was precisely controlled by adjusting the electrochemical deposition potential, which was critical to modulate the size and preferential orientation of the crystal growth along the (110) direction, and thus to improve the TE properties of the fabricated flexible TE generator (FTEG) (Fig. 6b). $\mathrm{A} \mathrm{Bi}_{2} \mathrm{Te}_{3}$ nanocrystal film prepared under a potential of $0.02 \mathrm{~V}$ (Fig. 6a) showed a high $\sigma\left(691 \mathrm{~S} \mathrm{~cm}^{-1}\right)$ with a maximum $P F$ of $1473 \mu \mathrm{W} \mathrm{m}{ }^{-1} \mathrm{~K}^{-2}$, which is the highest among the $\mathrm{Bi}_{2} \mathrm{Te}_{3}$ films prepared by the electrodeposition methods. Integrating it with an n-type $\mathrm{Bi}_{2} \mathrm{Te}_{3}$ FTEG, 

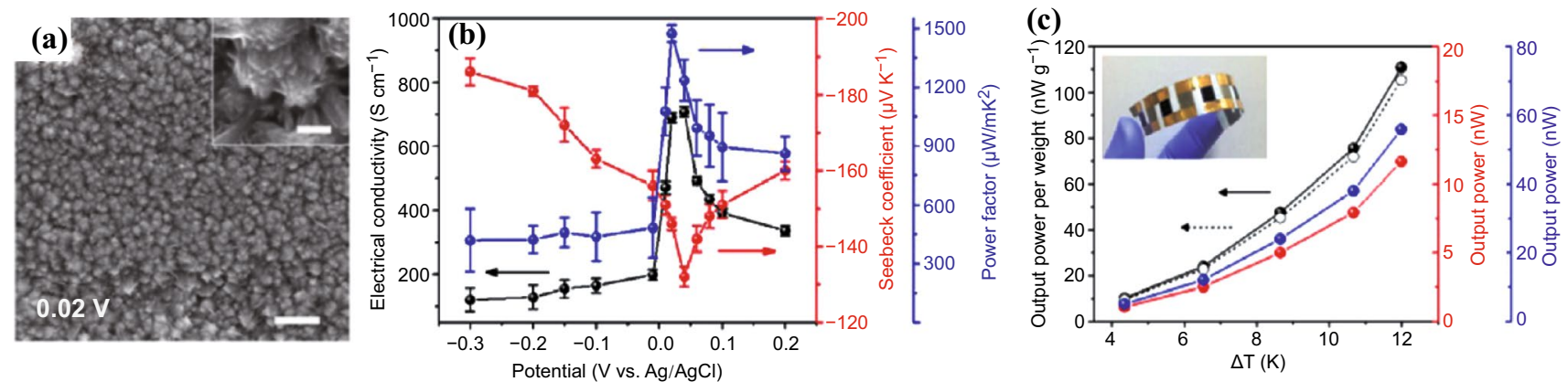

Fig. 6 a $\mathrm{SEM}$ image of electrodeposited $\mathrm{Bi}_{2} \mathrm{Te}_{3}$ nanocrystals at a potential of $0.02 \mathrm{~V}$ (scale bar: $500 \mathrm{~nm}$ ). b Corresponding $\sigma$ (black circle), $\mathrm{S}$ (red circle), and PF (blue circle) of the flexible TE generators (FTEGs) with $\mathrm{Bi}_{2} \mathrm{Te}_{3}$ films deposited at different potentials (V vs. $\mathrm{Ag} / \mathrm{AgCl}$ ). $\mathrm{c}$ Output power per weight of 1-couple (filled black circle) and 5-couples (open black circle) and output power of 1-couple (red circle) and 5-couples (blue circle) of the pn-FTEGs over a temperature gradient $(\Delta \mathrm{T})$. Adapted with permission from Ref. [96]. (Color figure online)

a prototype of a p-n-type flexible TEG (pn-FTEG) was prepared using a p-type polymer, poly(3,4-ethylenedioxythiophene)s. The pn-FTEG (5-couples) generated an output voltage of $5 \mathrm{mV}$ at $\Delta \mathrm{T}=12 \mathrm{~K}$ with a high output power of $105 \mathrm{nW} \mathrm{g}^{-1}$ (Fig. 6c). Very recently, Zhao et al. also prepared micrometer-thick $\mathrm{Bi}_{2} \mathrm{Te}_{3}$ nanocrystal films using the electrochemical deposition process [97]. The optimum $P F$ of as-grown $\mathrm{Bi}_{2} \mathrm{Te}_{3}$ films was achieved by shortening the period of the electrochemical deposition and introducing a photon-based rapid annealing process for the material postcrystallization. Compared with single crystalline or vacuum deposited $\mathrm{Bi}_{2} \mathrm{Te}_{3}$ films, the electronic transportations of the electrochemically deposited $\mathrm{Bi}_{2} \mathrm{Te}_{3}$ are more influenced by the carrier scatterings by the grain boundaries and lattice defect. In 2019, Nguyen et al. reported the synthesis of the gold nano-particles-bismuth telluride composites using the electrochemical co-deposition, and significant improvement in TE properties was achieved [98]. The composite with 5 $\mathrm{wt} \%$ of $5 \mathrm{~nm}$-diameter gold nanoparticles showed a highest absolute $S$ of $\sim 380 \mu \mathrm{V} \mathrm{K}^{-1}$, a low $\kappa$ of $\sim 0.5 \mathrm{~W} \mathrm{~m}^{-1} \mathrm{~K}^{-1}$ and a high ZT of $\sim 0.62$ at room temperature.

\subsubsection{D-Nanoinclusion}

The enhancement in TE properties of nanostructures by lowering the $\kappa$ is often off-set by the concurrent deterioration of the $\sigma$. As such, selectively lowering the $\kappa$ without compromising with the $\sigma$ remains a challenge. One possible way is to embed nanoparticles (particularly metal nanoparticles) with controlled sizes into a bulk matrix to increase $Z T$ values. The advantage of incorporating nanoparticles into
TE materials is able to reduce the $\kappa_{\mathrm{L}}$ due to the interfaces scattering of heat-carrying phonons, and it simultaneously enhances the $S$ via the electron energy filtering effect caused by the scattering of electrons on the band bending at the interfaces between nanoinclusions and the semiconductor host. The enhanced $S$ could compensate for the reduction in the $\sigma$ to some extent, thus maintaining the $P F$ at the similar level. The nanoinclusion composite structures have been generally prepared by the ball milling of metal $(\mathrm{Ag}, \mathrm{Au}, \mathrm{Cu}$, $\mathrm{Zn})$ or ceramic $\left(\mathrm{ZrO}_{2}, \mathrm{SiC}\right)$ nanoparticles with $\mathrm{TE}$ raw materials as the matrix phase [99-106]. However, such top-down processes for fabricating the low-dimensional structures are relatively expensive and time-consuming, probably not feasible for large-scale synthesis. Furthermore, enhancement in TE performance may be limited by the inhomogeneous distribution and aggregation of nanoparticles within the matrix.

In 2014, Sun and co-workers demonstrated the first bottom-up preparation of textured n-type $\mathrm{Bi}_{2} \mathrm{Te}_{2.7} \mathrm{Se}_{0.3}$ thin films with content-adjustable Pt nanoinclusions by the pulsed laser deposition [107]. Addition of Pt nanoinclusions resulted in a higher in-plane $P F$ based on the simultaneous increase in both the $\sigma$ and absolute $S$. The $P F$ of the optimized nanocomposite thin film reached $3.51 \times 10^{-3} \mathrm{~W} \mathrm{~m}^{-1} \mathrm{~K}^{-2}$ at room temperature, which is a more than $20 \%$ enhancement as compared to the single phase $\mathrm{Bi}_{2} \mathrm{Te}_{2.7} \mathrm{Se}_{0.3}$ thin film.

In 2015, Zhang et al. developed a facile and robust bottom-up chemical route to synthesize silver nanoparticles (AgNPs)-dispersed $\mathrm{Bi}_{2} \mathrm{Te}_{3}$ composites with a hierarchical two-phased heterostructure, in which the $\mathrm{Bi}_{2} \mathrm{Te}_{3}$ nanopowder was prepared by the surfactant-mediated hydrothermal method and AgNPs $(60 \mathrm{~nm})$ were obtained by using polyol 
reduction of silver nitrate, respectively, followed by the ultrasonic dispersion treatment and the SPS process [108]. The results clearly demonstrated that uniformly-dispersed AgNPs could lead to (1) growth-suppression of $\mathrm{Bi}_{2} \mathrm{Te}_{3}$ grains, (2) the introduction of nanoscale precipitates, and (3) the formation of new interfaces with $\mathrm{Bi}_{2} \mathrm{Te}_{3}$ matrix, leading to a hierarchical two-phased hetero-structure (Fig. 7a, b), which caused the intense scattering of phonons with multiscale mean free paths (Fig. 7c) and therefore significantly reduced the $\kappa_{\mathrm{L}}$. Meanwhile the improved $P F$ is maintained because of the Ag's low-energy electron filtering and superior electrical transport. A maximum $Z T$ value of 0.77 was obtained at $475 \mathrm{~K}$ from the bulk $\mathrm{Bi}_{2} \mathrm{Te}_{3}$ dispersed with 2.0 vol $\%$ AgNPs, which was significantly enhanced by $304 \%$ compared with that of the pristine bulk $\mathrm{Bi}_{2} \mathrm{Te}_{3}$.

In 2016, Ibanez reported the preparation of consolidated yet nanostructured TE materials based on a straightforward and versatile strategy involving bottom-up assembly of colloidal nanocrystals (Fig. 7d) [109]. PbS-Ag nanocomposites were prepared by mixing cubic $\mathrm{PbS}$ nanocrystals (ca. $11 \mathrm{~nm}$ ) with spherical Ag nanocrystals (ca. $3 \mathrm{~nm}$ ), followed by the removal of solvent through evaporation. Annealing was then performed to remove residual organic compounds, after which the resultant powdered nanocrystal blend was hot-pressed into pellets. PbS-Ag nanocomposites showed a highly homogeneous distribution of Ag nanodomains at the interfaces of $\mathrm{PbS}$ grains, as evidenced by high-resolution transmission electron microscope (HRTEM) (Fig. 7e). Ag nanodomains in the nanocomposites not only blocked phonon propagation, but also supplied electrons to the $\mathrm{PbS}$ host semiconductor and reduced the energy barriers between $\mathrm{PbS}$ crystal domains (Fig. 7f). The resultant nanocomposites therefore exhibited a reduced $\kappa$ and a higher charge carrier concentration and mobility compared to pure $\mathrm{PbS}$ nanomaterial. The $\sigma$ of the composites can reach up to $660 \mathrm{~S} \mathrm{~cm}^{-1}$ with a $\mathrm{Ag}$ concentration of above $4 \mathrm{~mol} \%$. The simultaneous combination of an outstanding $\sigma$, a relatively large $S$, and a reduced $\kappa$ contributed to a $Z T$ up to 1.7 at $850 \mathrm{~K}$.

Apart from the highly conductive metal nanoinclusions [110, 111], Lim et.al. has recently introduced nonmetal ( $\mathrm{Te}$ or $\mathrm{Se}$ ) nanodomains into a silver selenide matrix through solution blending of $\mathrm{Ag}_{2} \mathrm{Se}$ nanoparticles with $\mathrm{Te}$
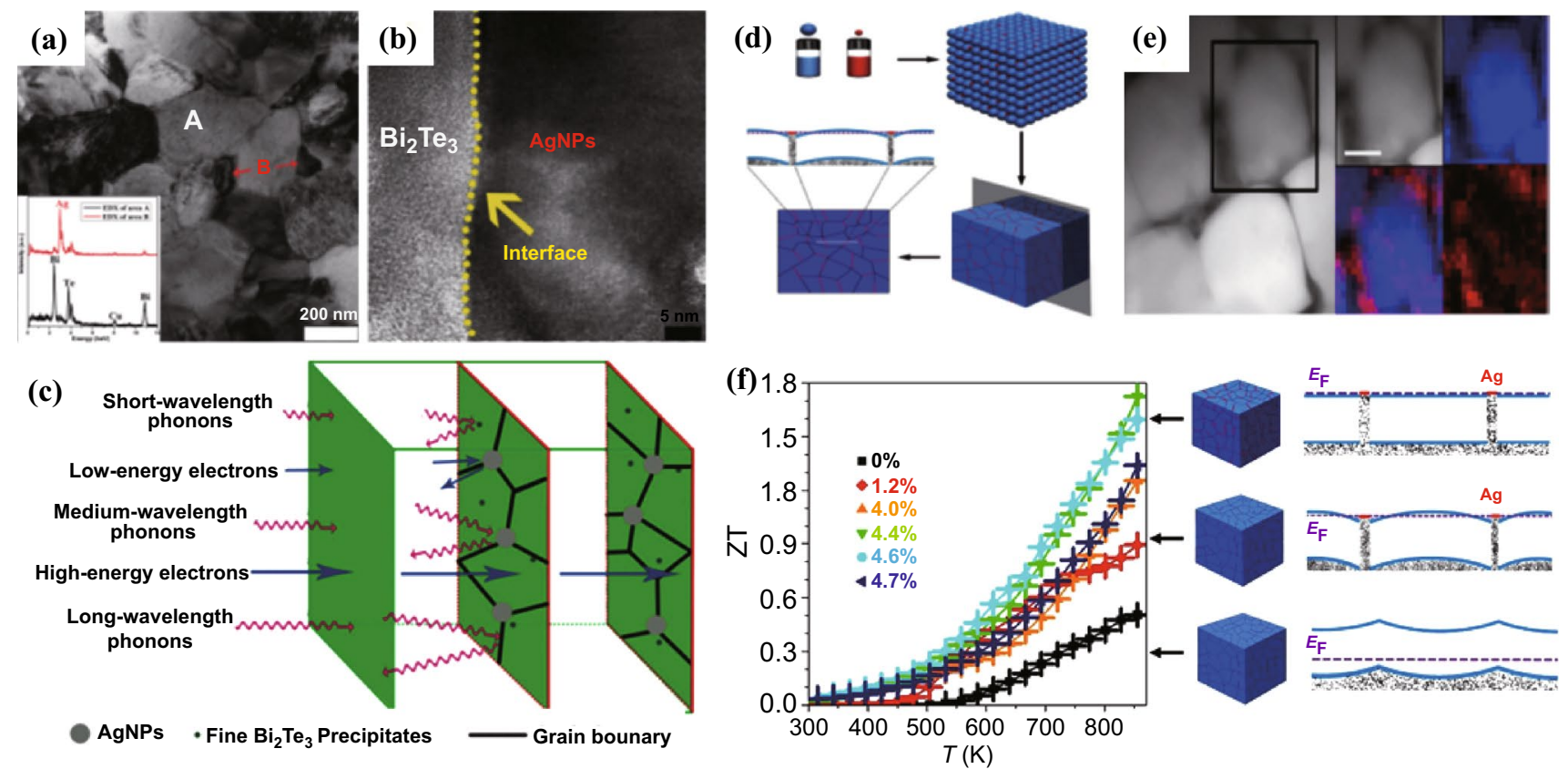

Fig. 7 a Low magnification TEM image of the bulk $\mathrm{Bi}_{2} \mathrm{Te}_{3}$ nanocomposite with 5.0 vol\% AgNPs nanocomposites. b HRTEM image showing the newly-built interface between the $\mathrm{Bi}_{2} \mathrm{Te}_{3}$ matrix and AgNPs. c Schematic drawing of a hierarchical two-phased heterostructure, showing the strong scattering of phonons with multiscale mean free paths along with the energy filtering effect. $\mathbf{d}$ Bottom-up assembly process to produce $\mathrm{PbS}-\mathrm{Ag}$ TE nanocomposites from the assembly of $\mathrm{PbS}$ (blue) and $\mathrm{Ag}$ (red) NCs, and the corresponding band alignment of the resulting nanocomposite. e HRTEM and elemental EDX mapping of the PbS-Ag $4.4 \mathrm{~mol} \%$ nanocomposite. f Figure of merit and schematic representation of the electron energy band alignment in $\mathrm{PbS}-\mathrm{Ag}$ nanocomposite. Adapted with permission from Refs. [108, 109]. (Color figure online) 
or Se nanorods before powder consolidation [112]. Different from the injection of conductive metal nanoinclusions that can lead to both an enhanced $\sigma$ and a lower $S$, the injection of a reduced concentration of charge carriers into a doped semiconductor could cause a band bending that promotes electron filtering. Interfacial energy filtering effects resulted in remarkable improvement in the $S$ being recorded for nanocomposite with $5 \mathrm{wt} \%$ Te nanoinclusion without significantly compromising with the $\sigma$. This nanocomposite displayed an improved average $Z T$ value of 0.84 in the temperature range of 300-400 K, higher than most of previously reported bulk $\mathrm{Ag}_{2} \mathrm{Se}$.

\subsection{D Nanowires/Nanofibers/Nanotubes}

The $Z T$ values of TE materials can be improved by introducing 1D nanostructures by (1) increasing $P F$ through the quantum confinement and/or energy filtering effect, or (2) reducing the $\kappa_{\mathrm{L}}$ via the enhanced phonon scattering [80, $113,114]$. The reduction in dimensionality from 3D bulks to $1 \mathrm{D}$ results in an enhancement in the electronic density of states at the energy band edges and thus causes an increase in the $P F$. In this section, we will introduce the bottom-up methods for preparation of semiconductor NWs and their structure-property relationships will be summarized.

\subsubsection{Solution-phase Synthesis}

Over the past decade, solution phase template-directed synthesis has been widely employed for the preparation of 1D metal chalcogenide NWs. In 2011 Wang et al. first reported a synthetic method for the controlled formation of ultrathin Te NWs using polyvinylpyrrolidone (PVP) surfactant. The as-prepared Te NWs served as sacrificial templates to facilitate the synthesis of highly uniform $\mathrm{Bi}_{2} \mathrm{Te}_{3} \mathrm{NWs}$ with a diameter of 15-17 nm and a length of tens of micrometers. The reaction was performed in triethylene glycol solution by adding Bi precursor and hydrazine to Te NWs at $200{ }^{\circ} \mathrm{C}$ and atmospheric pressure [115]. The formation of $\mathrm{Bi}_{2} \mathrm{Te}_{3} \mathrm{NWs}$ was demonstrated to be a result of the Kirkendall effect and Ostwald ripening (Fig. 8a). Using a similar method, Zhang et al. also prepared n-type ultrathin $\mathrm{Bi}_{2} \mathrm{Te}_{3} \mathrm{NWs}$ with an average diameter of $8 \mathrm{~nm}$ in ethylene glycol solution with a high yield up to $93 \%$ [116]. The as-prepared $\mathrm{Bi}_{2} \mathrm{Te}_{3} \mathrm{NWs}$ were then compressed to bulk pellets via the SPS process and was found to exhibit a high $Z T$ value of 0.96 at $380 \mathrm{~K}$, attributing to the increased phonon scattering taking place at NW boundaries and hence resulting in a notable decrease in the $\kappa$. Following this method, different kinds of binary telluride NWs with small diameters, including PbTe [117], CdTe [118], $\mathrm{Cu}_{2} \mathrm{Te}$ [119], and $\mathrm{Ag}_{2}$ Te NWs [117], have been

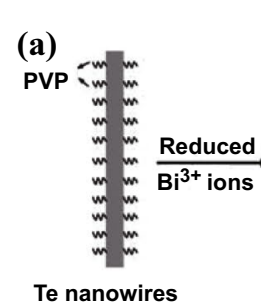

(b)

b) 'Wire' to 'barbell' Tellurium nanowire

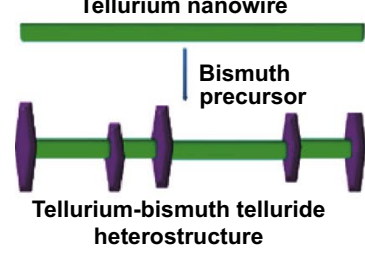
heterostructure

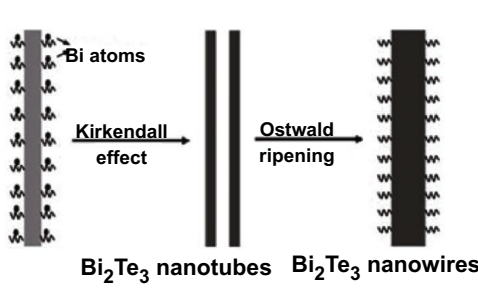

(c)

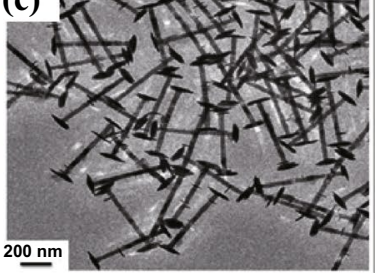

(d)

$\mathrm{i}$
CdS NW

ii $\mathrm{CdS} / \mathrm{Cu}_{2} \mathrm{~S}$ core/shell NW

\section{iii}

$\mathrm{CdS} / \mathrm{Cu}_{2} \mathrm{~S}$ superlattice NW

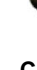

$\mathrm{Cu}^{+}$

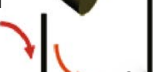

$\downarrow \rightarrow \mathrm{Cd}^{2+}$

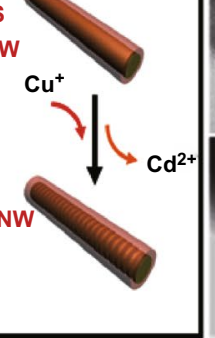

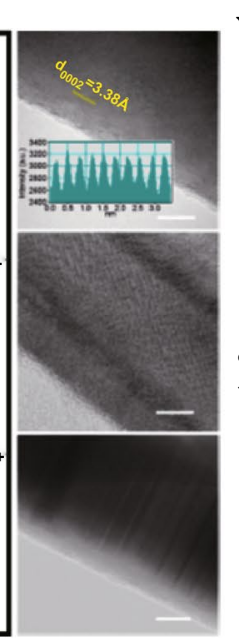

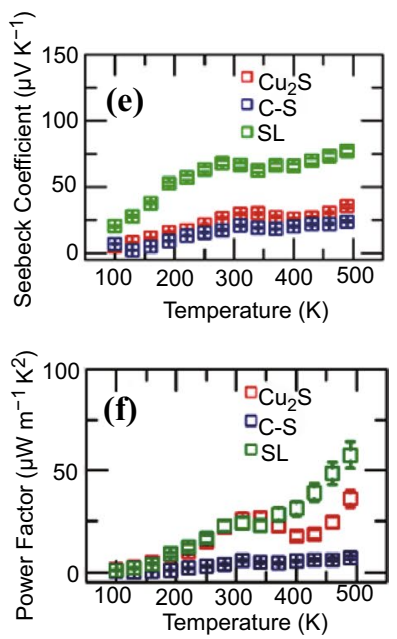

Fig. 8 a Schematic illustrations of the formation mechanism of the $\mathrm{Bi}_{2} \mathrm{Te}_{3} \mathrm{NWs}$ via a template-assisted solution phase process. b Schematic of tellurium NW formed in the first step and tellurium-bismuth telluride heterostructure after adding bismuth precursor in the second step. $\mathbf{c}$ TEM image of the $\mathrm{Te}-\mathrm{Bi}_{2} \mathrm{Te}_{3}$ heterostructured NWs. d Schematic diagram and corresponding TEM images (scale bar: $5 \mathrm{~nm}$ ) of phase evolution from pristine $\mathrm{CdS} \mathrm{NW}$ to $\mathrm{CdS} / \mathrm{Cu}_{2} \mathrm{~S}$ core-shell $\mathrm{NW}$, and eventually to $\mathrm{CdS} / \mathrm{Cu}_{2} \mathrm{~S}$ superlattice $\mathrm{NW}$. Temperature dependence of e thermopower and f power factor of $\mathrm{Cu}_{2} \mathrm{~S}$ nanowire, $\mathrm{CdS} / \mathrm{Cu}_{2} \mathrm{~S}$ core-shell nanowires $\left(\mathrm{C}-\mathrm{S}\right.$ ) and $\mathrm{CdS} / \mathrm{Cu}_{2} \mathrm{~S}$ superlattice nanowires (SL). Adapted with permission from Refs. [115, 120, 121] 
prepared via a solution phase synthesis. Solution phase synthesis can therefore be potentially translatable for low-cost, large-scale synthesis of materials for TE applications.

Compared with binary alloys, ternary alloys exhibit greater potential in tuning the band gap, elemental composition, charge-carrier density and conductivity, making them more promising for TE materials. In 2016, Zhou et al. reported a general synthesis approach to prepare ultrathin ternary metal chalcogenide NWs [122] involving the synthesis of ultrathin $6 \mathrm{~nm}$ diameter Te NWs, which served as precursors to fabricate $\mathrm{Te}_{x} \mathrm{Se}_{1-x} \mathrm{NWs}$ with tunable aspect ratios. The as-prepared $\mathrm{Te}_{x} \mathrm{Se}_{1-x} \mathrm{NWs}$ could then be converted into a series of ternary alloyed metal- $\mathrm{Te}_{x} \mathrm{Se}_{1-x} \mathrm{NWs}$ by injecting appropriate metal precursors under specific conditions. A series of ultrathin ternary alloyed NWs, including $\mathrm{Bi}_{2} \mathrm{Te}_{x} \mathrm{Se}_{3-x}, \mathrm{Ag}_{2} \mathrm{Te}_{x} \mathrm{Se}_{1-x}, \mathrm{Cu}_{1.75} \mathrm{Te}_{x} \mathrm{Se}_{1-x}, \mathrm{CdTe}_{x} \mathrm{Se}_{1-x}$, and $\mathrm{PbTe}_{x} \mathrm{Se}_{1-x}$ were synthesized and characterized for the first time, with controllable $\mathrm{Te} / \mathrm{Se}$ ratios. Among these, $\mathrm{Bi}_{2} \mathrm{Te}_{2.7} \mathrm{Se}_{0.3} \mathrm{NW}$-based bulk material exhibited outstanding TE performance with a high $P F$ of $1023 \mu \mathrm{W} \mathrm{m}{ }^{-1} \mathrm{~K}^{-2}$ and a $Z T$ of 0.75 at $320 \mathrm{~K}$.

In 2012, Wu's group demonstrated a design principle to prepare new categories of telluride-based TE NW heterostructures through solution-phase reactions [120]. The catalyst-free synthesis started with the preparation of Te NWs, followed by the growth of $\mathrm{Bi}_{2} \mathrm{Te}_{3}$ nanoplates on the Te NW tips and bodies, yielding $\mathrm{Te}-\mathrm{Bi}_{2} \mathrm{Te}_{3}$ "barbell" $\mathrm{NW}$ heterostructures with a narrow diameter $(\sim 36 \mathrm{~nm})$ and length distribution as well as a rough control over the density of the hexagonal $\mathrm{Bi}_{2} \mathrm{Te}_{3}$ nanoplates by varying the reaction conditions (Fig. 8b, c). The hot-pressed nanostructured bulk pellets of the $\mathrm{Te}-\mathrm{Bi}_{2} \mathrm{Te}_{3}$ heterostructure showed a largely enhanced $S$ (up to $608 \mu \mathrm{V} \mathrm{K}^{-1}$ at $300 \mathrm{~K}$ ) and a greatly reduced $\kappa\left(0.365 \mathrm{~W} \mathrm{~m}^{-1} \mathrm{~K}^{-1}\right.$ at $\left.300 \mathrm{~K}\right)$ due to the energy filtering effect occurring at the grain-grain interfaces and the phonon scattering at the NW-NW, NW-plate, and plate-plate interfaces. In the follow-up study, these "barbell"-like $\mathrm{Te}-\mathrm{Bi}_{2} \mathrm{Te}_{3} \mathrm{NWs}$ were further converted to other telluride-based compositional modulated NW heterostructures such as $\mathrm{PbTe}-\mathrm{Bi}_{2} \mathrm{Te}_{3}$ and $\mathrm{Ag}_{2} \mathrm{Te}-\mathrm{Bi}_{2} \mathrm{Te}_{3}$, which displayed a high $Z T$ of 1.2 (at $620 \mathrm{~K}$ ) and 0.41 (at $400 \mathrm{~K}$ ), respectively [123, 124].

A low-cost solution process, the strain induced selective phase segregation technique, to produce superlattice nanostructures, was reported by Tang, et al. in a $\mathrm{CdS} / \mathrm{Cu}_{2} \mathrm{~S}$ system. Due to the energy filtering effect, the superlattice
NWs exhibited an improved $S$ without sacrificing the $\sigma$ [121]. The distinct interface formation energy at different $\mathrm{CdS}$ facets and the self-regulated strain energy relaxation at the $\mathrm{CdS}-\mathrm{Cu}_{2} \mathrm{~S}$ interface facilitated the conversion of $\mathrm{CdS} \mathrm{NW}$ into $\mathrm{CdS} / \mathrm{Cu}_{2} \mathrm{~S}$ core-shell structures (Fig. 8d). Their $S$ can be significantly enhanced (Fig. 8e) by the energy filtering effect which was favored by the junction formed at the $\mathrm{CdS}-\mathrm{Cu}_{2} \mathrm{~S}$ interface, while the $\sigma$ of the superlattice NWs was not greatly compromised, leading to greatly enhanced power factor at temperature higher than $400 \mathrm{~K}$ (Fig. 8f).

\subsubsection{Vapor-liquid-solid Growth}

The vapor-liquid-solid method (VLS) is widely used to grow 1D structures, such as NWs $[125,126]$. The synthetic procedures typically start with the deposition of metal catalyst on a substrate, which is then converted to liquid alloy droplets by adsorbing the precursor vapor component at a high temperature (Fig. 9a). Crystal growth through direct gas phase adsorption onto a solid surface is typically very slow. The VLS method therefore circumvents this by introducing a catalytic liquid alloy phase which can rapidly adsorb a vapor to a supersaturation level, allowing crystal growth to occur from nucleated seeds at the liquid-solid interface. The bottom-up growth of semiconductor NWs by the VLS method is able to precisely control their size, morphology, growth density, spatial distribution, composition as well as element doping.

In an early work, Li et al. fabricated VLS-grown individual single crystalline intrinsic $\mathrm{Si}$ NWs with diameters of 22, 37, 56, and $115 \mathrm{~nm}$ [127]. The size effects on the $\kappa$ of these individual NWs were studied (Fig. 9e). The $\kappa$ observed was lower than the bulk value, and the strong diameter dependence of the $\kappa$ in NWs was ascribed to the increased phonon-boundary scattering and possible phonon spectrum modification. Using the hybrid pulsed laser ablation and the VLS growth process, the same group also prepared single crystalline $\mathrm{Si} / \mathrm{SiGe}$ superlattice NWs (Fig. 9d) with diameters of 58 and $83 \mathrm{~nm}$ [128]. Compared with the pure Si NWs in which alloy scattering is suggested to be the dominant phonon scattering mechanism for the thermal transport, this study demonstrated that the NW boundary scattering played a role in reducing the $\kappa$. In 2011 and 2012, Kim and Park et al. synthesized 

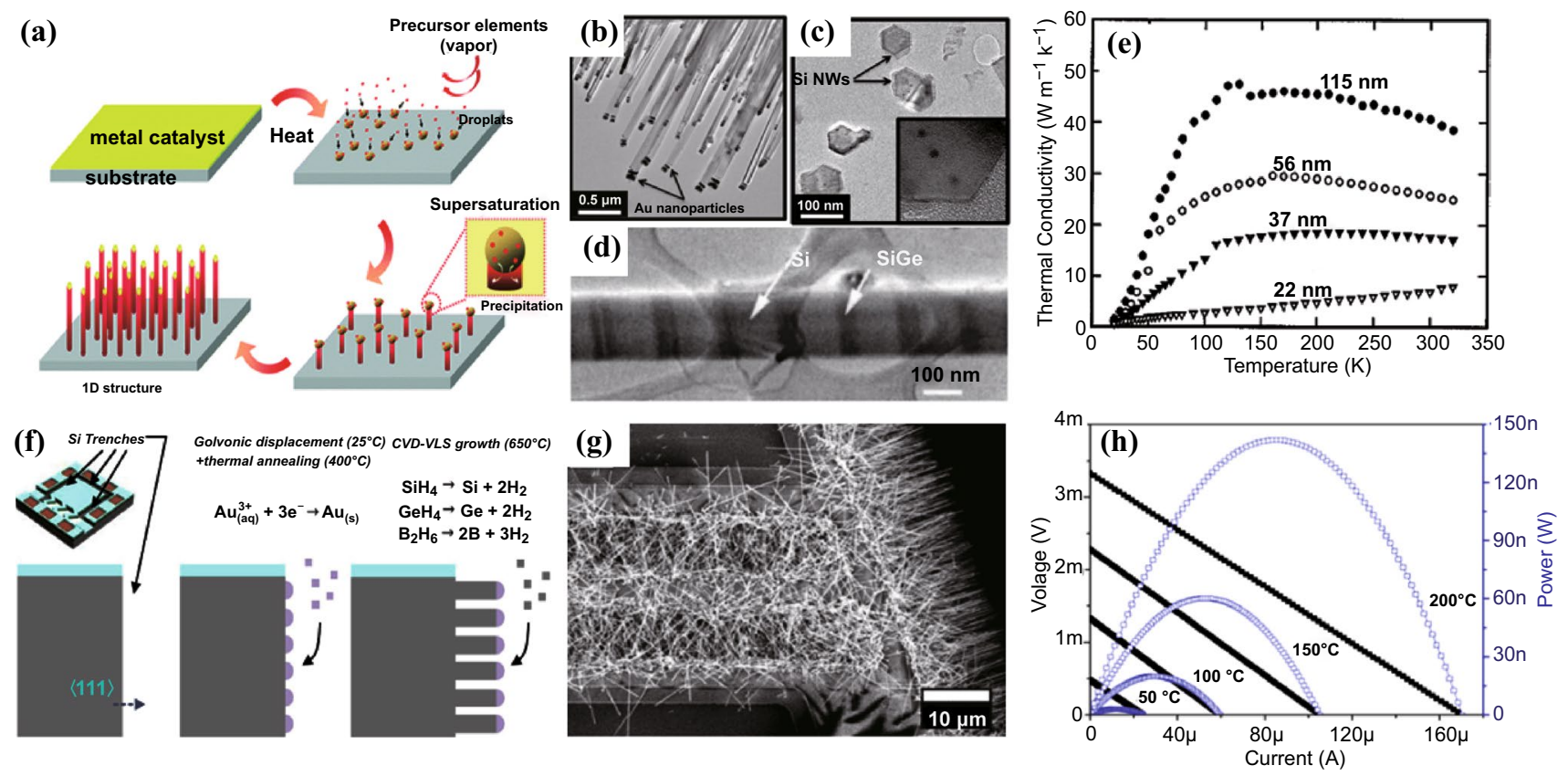

Fig. 9 a Schematic diagram of the synthesis of the NWs by VLS growth method. b TEM image of Si NW arrays showing gold nanoparticles on the tip. $\mathbf{c}$ Cross-section TEM image of silicon NWs showing hexagonal shape. d TEM image of Si/SiGe superlattice NWs. e Measured thermal conductivity of different diameter Si NWs. f Schematic steps for CVD-VLS growth of boron-doped SiGe NWs and integration in $\mu$ TEGs. $\mathbf{g}$ Topview SEM image of SiGe NWs selectively grown in Si exposed parts. $\mathbf{h}$ Current-voltage and current-power curves obtained from SiGe NWs based $\mu$ TEGs with three trenches for different hot plate temperatures. Adapted with permission from Refs. [127-129]

VLS-grown rough $\mathrm{Si}$ and $\mathrm{Si}_{0.96} \mathrm{Ge}_{0.04} \mathrm{NWs}$ with various surface roughness and diameters with the assistance of different catalysts $[130,131]$. It was found that the surface roughness affected the $\kappa$ more significantly than the diameter of the NWs. Theoretical analysis reveals that the surface roughness scattering affects mid-wavelength phonons, whereas the phonon boundary scattering affects long-wavelength phonons and the alloy scattering affects short-wavelength phonons.

The characteristics of CVD-VLS process enables the convenient creation of microscale TE devices with control over photonic, electronic, and thermal properties. In 2013, Davila et al. first fabricated dense arrays of well-oriented and size-controlled Si NWs (Fig. 9b, c) obtained from the CVD-VLS process and implemented them into microfabricated structures to make a planar unileg TE microgenerator (uTEGs) [132, 133]. The average diameter and the length of the Si NWs are $100 \mathrm{~nm}$ and $10 \mu \mathrm{m}$, respectively. The resulting TEG can generate power densities of $1.44 \mathrm{~mW}$ $\mathrm{cm}^{-2}$ and $9 \mu \mathrm{W} \mathrm{cm}{ }^{-2}$ under temperature differences of 300 and $27 \mathrm{~K}$, respectively. In 2017, Hill et al. synthesized VLS-grown uniform, linear, and degenerately boron- and phosphorous-doped Si NW superlattices with abrupt transitions between p-type, intrinsic, and n-type segments [134]. Recently, Noyan et al. reported the bottom-up growth of SiGe NW arrays by means of CVD-VLS and their monolithic integration into TE microgenerators (Fig. 9f-h) [129]. Densely aligned boron-doped SiGe NWs with a diameter of $64 \pm 11 \mathrm{~nm}$, a length of $10 \mu \mathrm{m}, 30 \%$ Ge content, and doping of $\sim 10^{20} \mathrm{~cm}^{-3}$ were grown simultaneously and integrated via the gold-catalyzed CVD-VLS approach in devices with different numbers of micro-trenches. A three-trench single thermocouple placed on a $200{ }^{\circ} \mathrm{C}$ heat source could achieve a maximum power of $142 \mathrm{nW}$ which is equivalent to a power density of $7.1 \mu \mathrm{W} \mathrm{cm}{ }^{-2}$, demonstrating the great potential of the as-prepared material for energy harvesting from waste heat.

\subsubsection{Template-Assisted Electrodeposition Method}

Template-assisted electrodeposition is the most convenient method for synthesizing NWs with controlled stoichiometry, size, morphology and crystallinity [135]. Moreover, it is also cost-effective and scalable for applications. For 
conventional TE materials based on chalcogenide semiconductors, electrodeposition methods are widely employed for creating high-aspect-ratio NWs using hard templates with enormous 1D nanochannels. The two most common templates to obtain NWs by electrodeposition are anodic aluminum oxide (AAO) and polycarbonate (PC) membranes with different pore sizes (ranging from tens of nanometers to hundreds of nanometers) and template thickness. The AAO membranes are typically removed by chemical dissolution, while the PC membranes can be removed by either chemical dissolution or heat treatment in the air. The electrodeposition process can be conducted at three different modes: constant potential, current density and pulsed electrodeposition, among which the pulsed electrodeposition can provide more uniform growth and higher crystallinity of NWs [136].

It is well known that $\mathrm{Bi}_{2} \mathrm{Te}_{3}$ can behave as an n-type or p-type semiconductor depending on its stoichiometry. The Bi-rich composition shows a p-type semiconductor with a positive $S$, while the Te-rich stoichiometric ratio is an n-type semiconductor with a negative $S$. During the past decade, tremendous efforts have been made to prepare Bi-Te NWs with tunable compositions, morphologies and crystallographic structures using the template-assisted electrodeposition method [137-144]. In 2017, Proenca and co-workers studied the effect of deposition applied potential on the morphology, stoichiometry and crystallinity of Bi-Te NWs using the AAO template [138]. The morphology and the Te\% content was found to be highly dependent on the deposition potential. X-ray diffraction measurements revealed that there was strong relationship between the material's crystallinity and the deposition potential, being monocrystalline at very low potentials, but almost completely amorphous at high potentials due to high growth rates. In the same year, Rojo et al. reported electrodeposited $\mathrm{Bi}_{2} \mathrm{Te}_{3} \mathrm{NWs}$ with 300, 52, 45, and $25 \mathrm{~nm}$ diameters using the AAO template (Fig. 10a-d), and in-depth study of how the $\kappa$ of $\mathrm{Bi}_{2} \mathrm{Te}_{3} \mathrm{NWs}$ was affected when reducing its diameter from an experimental and theoretical point of view [145]. The $\kappa$ was observed to decrease more than $70 \%$ (from $1.78 \pm 0.46$ to $0.52 \pm 0.35 \mathrm{~W} \mathrm{~K}^{-1} \mathrm{~m}^{-1}$ ) when the diameter of the NW was reduced one order of magnitude (from 300 to $25 \mathrm{~nm}$ ) (Fig. 10e). An increment of the phonon scattering is believed to be responsible for the reduction in the NWs' $\kappa$. As mentioned by the Kinetic-Collective model (KCM) [146], the reduction in the $\kappa$ is mainly caused by the alteration of the mean free path of the acoustic phonons due to the size confinement. Reeves et al. fabricated electrodeposited sub-10 $\mathrm{nm} \mathrm{Bi}_{2} \mathrm{Te}_{3} \mathrm{NW}$ arrays using novel silica-coated AAO templates for the pore confinement [137].
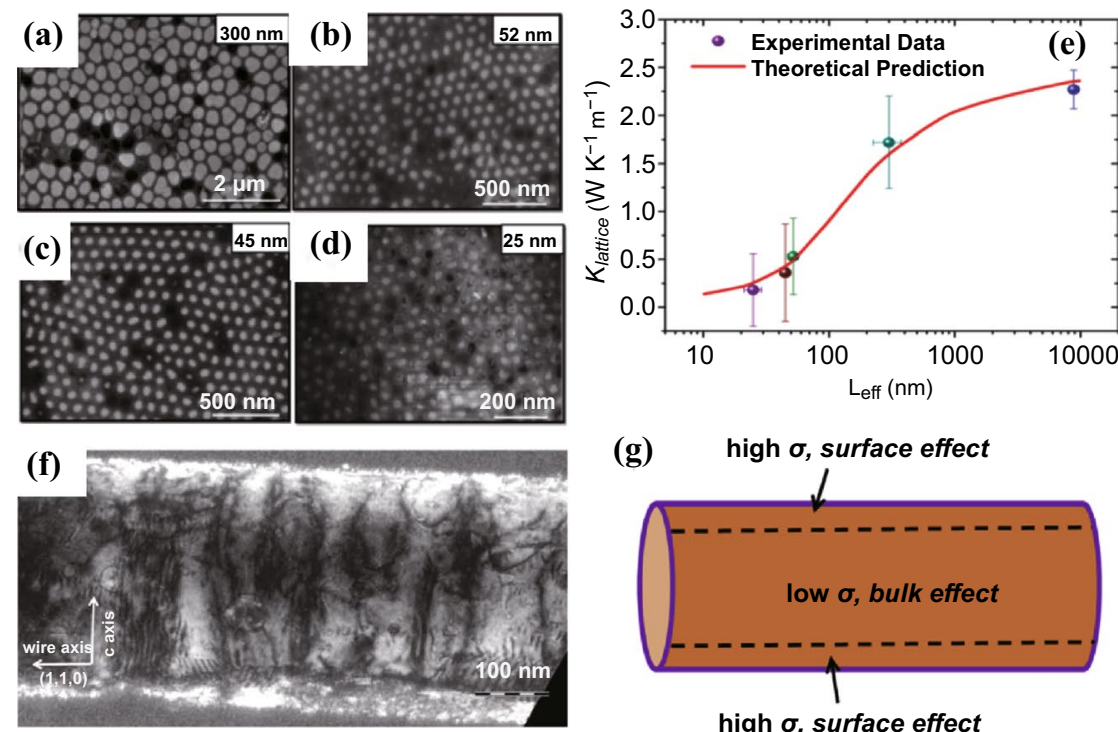

high $\sigma$, surface effect

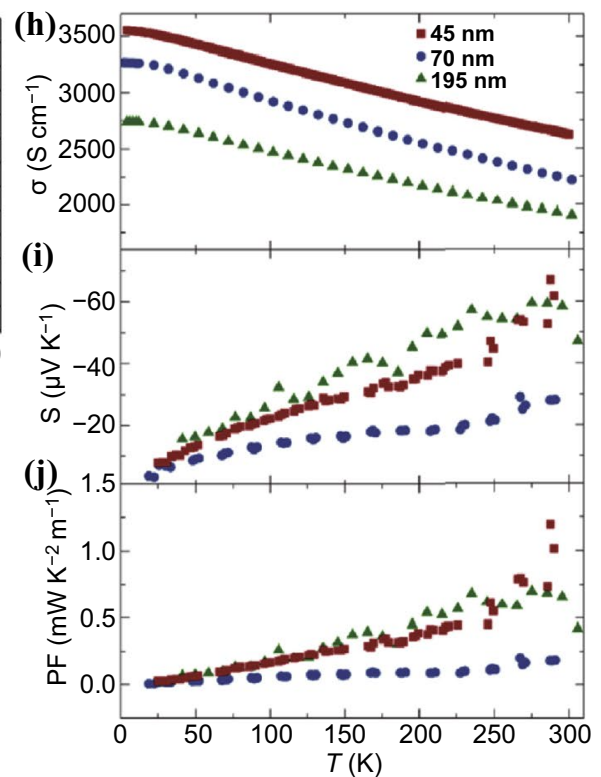

Fig. 10 SEM images of a $300 \mathrm{~nm}$, b $52 \mathrm{~nm}$, c $45 \mathrm{~nm}$ and d $25 \mathrm{~nm}$ average diameter $\mathrm{Bi}_{2} \mathrm{Te}_{3} \mathrm{NWs}$. e $\kappa_{\mathrm{L}}$ of the NWs versus diameters. f TEM image of a $\mathrm{Bi}_{2} \mathrm{Te}_{3-y} \mathrm{Se}_{y}$ ternary NW. $\mathrm{g}$ Schematic of the NW showing the contribution of the surface states with a high $\sigma$, whereas bulk part yields a low electrical conductivity and a high $S$. Temperature-dependent $\mathbf{h}$ electrical conductivity, $\mathbf{i}$ Seebeck coefficient and $\mathbf{j}$ power factor of $\mathrm{Bi}_{2} \mathrm{Te}_{3-y} \mathrm{Se}_{y}$ ternary NWs of three different diameters (45, 70 and $\left.195 \mathrm{~nm}\right)$. Adapted with permission from Refs. [137, 147] 
The obtained sub-10 nm NWs displayed a greatly increased electrical-to-thermal conductivity ratio as the pore diameter decreased.

Furthermore, it is well-known that $\mathrm{Bi}_{2} \mathrm{Te}_{3}$ can switch its n-type or p-type semiconductor response if it is doped with $\mathrm{Se}$ and $\mathrm{Sb}$, respectively. Various ternary NWs based on Sb-doped Bi-Te [136, 148-155] and Se-doped Bi-Te NWs [147, 149, 156, 157] have also been developed using the electrodeposition method. In 2013, Baßler et al. published p- and n-type single-crystalline NWs of bismuth antimony telluride and bismuth telluride selenide grown by the template-based millisecond pulsed electrochemical deposition in self-ordered $\mathrm{Al}_{2} \mathrm{O}_{3}$ membrane templates [149]. The as-grown NWs with a diameter of 80 and $200 \mathrm{~nm}$ were annealed in helium and tellurium atmosphere to reduce the crystal defects which led to higher TE performance. The PFs of the obtained $\mathrm{Bi}_{38} \mathrm{Te}_{55} \mathrm{Se}_{7}$ and $\mathrm{Bi}_{15} \mathrm{Sb}_{29} \mathrm{Te}_{56} \mathrm{NWs}$ reached 2820 and $1750 \mu \mathrm{W} \mathrm{K} \mathrm{K}^{-2} \mathrm{~m}^{-1}$, respectively, at room temperature, which are significantly higher compared to thin films as a result of the higher $\sigma$ of the 1D structure. In 2015, $\mathrm{Li}$ et al. reported the pulse-deposited of Bi-Sb-Te NWs which displayed more homogeneous element distribution and higher crystallinity compared to direct current (DC)deposited NWs [136]. The ZT of the pulse-deposited single $\mathrm{Bi}_{0.5} \mathrm{Sb}_{1.5} \mathrm{Te}_{3} \mathrm{NW}$ seached as high as 1.14 at $330 \mathrm{~K}$, which is approximately $54 \%$ higher than that of the DC-deposited ones. Kumar et al.also prepared single-crystalline, ternary n-type Bi-Te-Se NWs (Fig. 10f) with different nominal diameters of 45,70 , and $195 \mathrm{~nm}$ by electrodeposition in a nanostructured $\mathrm{Al}_{2} \mathrm{O}_{3}$ matrix [147]. The transport properties of individual NWs were measured, yielding the largest $\sigma\left(2620 \mathrm{~S} \mathrm{~cm}^{-1}\right.$ at room temperature) for the smallest NW (Fig. 10h). This behavior of the $\sigma$ might be attributed to the highly conductive surface states (Fig. 10g). Compared to bulk materials, relatively lower $S$ (up to $-60 \mu \mathrm{V} \mathrm{K} \mathrm{K}^{-1}$ at room temperature) was obtained for these NWs due to the existence of metallic surface states (Fig. 10h). The highest $P F\left(0.8 \mathrm{~mW} \mathrm{~K}^{-2} \mathrm{~m}^{-1}\right)$ was achieved with the $195 \mathrm{~nm} \mathrm{NWs}$ (Fig. 10i), which is suppressed compared to bulk values but higher than those of thin films.

\subsubsection{Electrospinning}

Electrospinning is a simple and versatile technique for producing continuous nanofibers from polymers and ceramics under a high electric field with controllable morphology, diameter, composition and orientation [158, 159]. In some early work, TE properties of oxide materials have been significantly improved when their bulk 3D dimensions are reduced to $1 \mathrm{D}$ nanoscale by the sol-gel based electrospinning. In 2010, Yin et al. fabricated nanocrystalline $\mathrm{Ca}_{3} \mathrm{Co}_{4} \mathrm{O}_{9}$ nanofibers with diameters around $350 \mathrm{~nm}$ using the sol-gel electrospinning process, which were consolidated into bulk ceramics by the SPS process [160]. The nanofiber-sintered ceramic with a much smaller grain size exhibited simultaneously enhanced $S, \sigma$ and thermal resistivity, resulting in $55 \%$ enhancement in $Z T$ (around 0.40 at $975 \mathrm{~K}$ ). In the same year, $\mathrm{Ma}$ et al. also reported TE nanocrystalline electrospun $\mathrm{NaCo}_{2} \mathrm{O}_{4}$ nanofibers with a grain size of as small as $10 \mathrm{~nm}$ [161], and $\mathrm{Xu}$ et al. produced $\mathrm{TE} \mathrm{La}_{0.95} \mathrm{Sr}_{0.05} \mathrm{CoO}_{3}$ nanofibers with a diameter of $\sim 35 \mathrm{~nm}$ by electrospinning with a greatly enhanced $S$ of $650 \mu \mathrm{V} \mathrm{K}^{-1}$ at room temperature [162].

Metal chalcongenide semiconductor nanofibers have also been synthesized using the electropinning technique combined with electrochemical reactions. In 2018, Park et al. first reported the large scale fabrication of a few millimeterlong lead telluride ( $\mathrm{PbTe}$ ) hollow nanofibers employing a three-step sequential process involving electrospinning, then electrodeposition and finally, cationic exchange reaction [163]. Electrodeposition of Te onto as-prepared electrospun Ag nanofibers possessing an ultra-long aspect ratio of 10,000 afforded silver telluride nanotubes, which then underwent a cationic exchange reaction in $\mathrm{Pb}\left(\mathrm{NO}_{3}\right)_{2}$ solution to obtain polycrystalline PbTe nanotubes with $100 \mathrm{~nm}$ average diameter and $20 \mathrm{~nm}$ wall thickness (Fig. 11a, b). The Ag-to- $\mathrm{Pb}$ ratio in the $\mathrm{Ag}_{x} \mathrm{Te}_{y}-\mathrm{PbTe}$ nanocomposites could be easily tuned during the cationic exchange reaction (Fig. 11c), which rendered good control over the TE properties of resulting 1D hollow nanofibers (Fig. 11d-f). The content of $\mathrm{Ag}$ ion led to the enhancement of TE properties in the $\mathrm{Ag}_{x} \mathrm{Te}_{y}-\mathrm{PbTe} 1 \mathrm{D}$ nanocomposite mats, which showed the highest value of $S$ of $433 \mu \mathrm{V} \mathrm{K}^{-1}$ at $300 \mathrm{~K}$ when the remained Ag content was $30 \%$. Zhang et al. also fabricated $\mathrm{PbTe}$ hollow nanofiber mats through combining electrospinning, followed by galvanic displacement reactions using electrospun cobalt nanofibers as the sacrificial material [164]. Through tuning the diameter of the sacrificial cobalt nanofibers as well as the electrolyte concentrations in the galvanic displacement reactions, $\mathrm{PbTe}$ hollow nanofibers with various dimensions, surface morphologies 

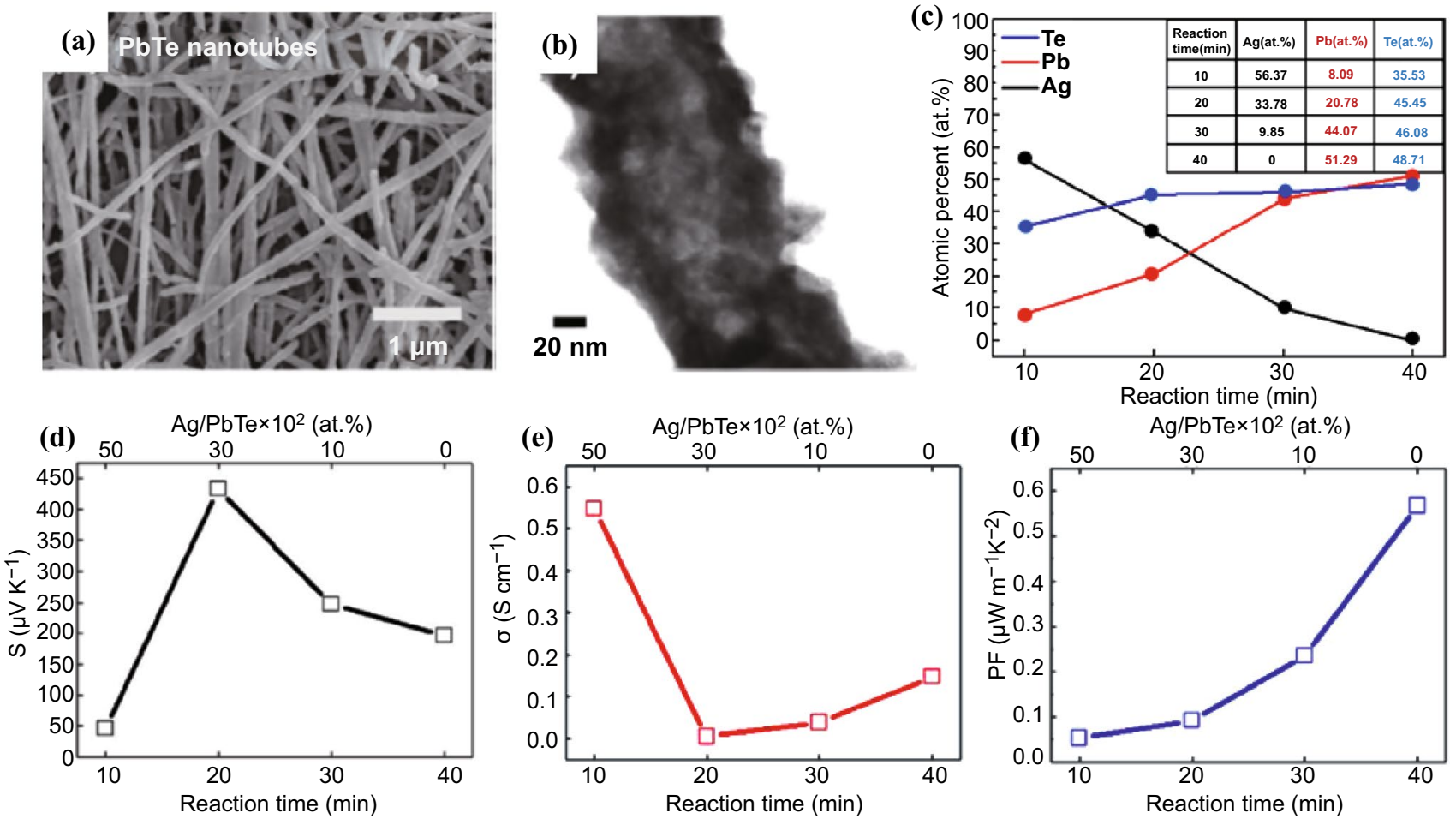

Fig. 11 a SEM and $\mathbf{b}$ TEM images of PbTe nanotubes fabricated via combined electrospinning, electrodeposition and cationic exchange reaction. c Stoichiometry ratio of different cation exchange reaction time from $\mathrm{Ag}_{2} \mathrm{Te}$ to PbTe. Different reaction time of $\mathbf{d}$ Seebeck coefficient, $\mathbf{e}$ electrical conductivity and $\mathbf{f}$ power factor of $\mathrm{PbTe}$, included $10-50 \%$ of $\mathrm{Ag}$ content in the $1 \mathrm{D} \mathrm{Ag}_{x} \mathrm{Te}_{y}-\mathrm{PbTe}$ composite nanotubes. Adapted with permission from Ref. [163]

and compositions were synthesized, demonstrating that both the quantum confinement and surface scattering effects displayed an additional degree of control over the TE properties within such polycrystalline tubular nanostructures.

\subsection{D Nanoflake/Nanosheet/Nanoplate}

Since the discovery of graphene through the mechanical exfoliation by scotch tape in 2004 [165], various structures of 2D materials have been designed and fabricated to facilitate its physical and electronic properties because of its unique and advantageous structural features. Unlike bulk materials, 2D structure can easily tune its charge concentration, carrier transition through its network or different layers although some parameters, especially the size and shape of 2D materials, are rather difficult to be controlled [166-168]. In order to prepare various 2D structures based on semiconducting materials, various top-down methods have been designed including exfoliation, ball milling [169, 170]. This section will provide an overview of various bottom-up approaches developed recently for preparation of semiconducting 2D nanomaterials for TE applications, including vacuum-based techniques and wet-chemical synthesis. The vacuum-based deposition methods are limited to fabrication of thin films with highest-level control of crystal quality and composition. Although high power factors can be achieved using these approaches, the main drawback lies in its high cost and low productivity. In comparison, wet-chemical synthesis is more cost-effective for massive production of both thin films and bulk materials. More importantly, wetchemical synthesis offers easy control over size, shape and composition of the 2D materials and it is also convenient to introduce dopants in the synthetic process to fine-tune and optimize the thermoelectric properties of the prepared 2D materials.

\subsubsection{Chemical Vapor Deposition}

2D layered IV-VI chalcogenides have attracted great attention for the electronic applications due to their band gaps 
that can facilitate the carrier movement in the 2D network. As a most representative example, $\mathrm{SnSe}$ has been widely studied for solar cell and optoelectronic devices [171, 172]. Owing to its layered structure, the $\kappa_{\mathrm{L}}$ of SnSe could be reduced significantly to as low as $0.2-0.3 \mathrm{~W} \mathrm{~m}^{1} \mathrm{~K}^{-1}$ at $800 \mathrm{~K}$ [173]. Meantime, SnSe has a semiconducting energy gap of $\approx 0.86 \mathrm{eV}$ and in general a very low $\sigma\left(10^{-5}\right.$ to $0.1 \mathrm{~S}$ $\mathrm{cm}^{-1}$ ). Therefore, CVD has been used to introduce extra elements to enhance the carrier concentration so as to improve its $\sigma$ [174-176]. However, doping certain elements to $\mathrm{SnSe}$ via vapor deposition is rarely studied because of its extreme growth process conditions.

Recently, Gao et al. reported a facile CVD approach to grow and dope SnSe nanoflakes, and fabricate the nanostructured thin films (Fig. 12) [177]. The nanostructured structure belongs to the Pnma space group with a layered structure along a-axis, enabling the CVD growth-based nanostructure favorable towards one direction. This is of great interest for TE performance as it potentially moves the carrier towards one particular direction. The CVD growth of SnSe nanoflakes was conducted using $99.999 \%$ SnSe powder on the Si wafer with $300 \mathrm{~nm}$ thick silicon oxide in the temperature range of 750 to $550 \mathrm{~K}$. Figure 12c, d show the SnSe nanoflakes formed on the surface of the substrate, illustrating that the uniform of nanoflakes were generated during the process.

Upon obtaining the nanoflakes, Gao's group prepared $\mathrm{SnSe}_{1.13}$ and $\mathrm{SnSe}_{0.75}$ aiming to study how the ratio difference affects the TE performance [177]. Se deficiency in $\mathrm{SnSe}_{0.75}$ contributed to a much higher hole density with a lower hole mobility when compared with $\mathrm{SnSe}_{1.13}$, showing poorer TE performance. In the case of $\mathrm{SnSe}_{1.13}$, the obtained highest $\sigma$ and the $S$ were about $2.5 \mathrm{~S} \mathrm{~cm}^{-1}$ and $300 \mu \mathrm{V} \mathrm{K}^{-1}$, making the $P F$ at the level of $0.16 \mu \mathrm{W} \mathrm{cm} \mathrm{cm}^{-1} \mathrm{~K}^{-2}$ (Fig. 12b), which is about $5 \%$ of the $P F$ of SnSe single crystals. The low $P F$ value could be due to the low carrier mobility and carrier concentration. The same group also doped Ag atoms into SnSe at different ratios and observed that a doping level at about $1 \%$ could significantly improve the $\sigma$ and $S$. The highest $\sigma$ and $S$ could be up to $4 \mathrm{~S} \mathrm{~cm}^{-1}$ and $370 \mu \mathrm{V} \mathrm{K}^{-1}$ respectively, making the $P F$ to be improved to $0.66 \mu \mathrm{W} \mathrm{cm}{ }^{-1} \mathrm{~K}^{-2}$, four times of the value of undoped sample. Besides, the synthesized SnSe samples could possess a low $\kappa$, which could further improve the $Z T$ value.

\subsubsection{Molecular Beam Epitaxy}

Molecular beam epitaxy (MBE) is widely used to generate 2D layer nanostructures [178-180] on the solid layer of materials, such as silicon $[181,182]$. In this area, the preparation of a 2D layer of semiconducting metals or alloys becomes very attractive. Mori et al. fabricated $\mathrm{Mg}_{2} \mathrm{Sn}$ (111) thin film on a sapphire c-plane using pure $\mathrm{Mg}$ and Sn elements under MBE condition [183]. Below $250{ }^{\circ} \mathrm{C}$,

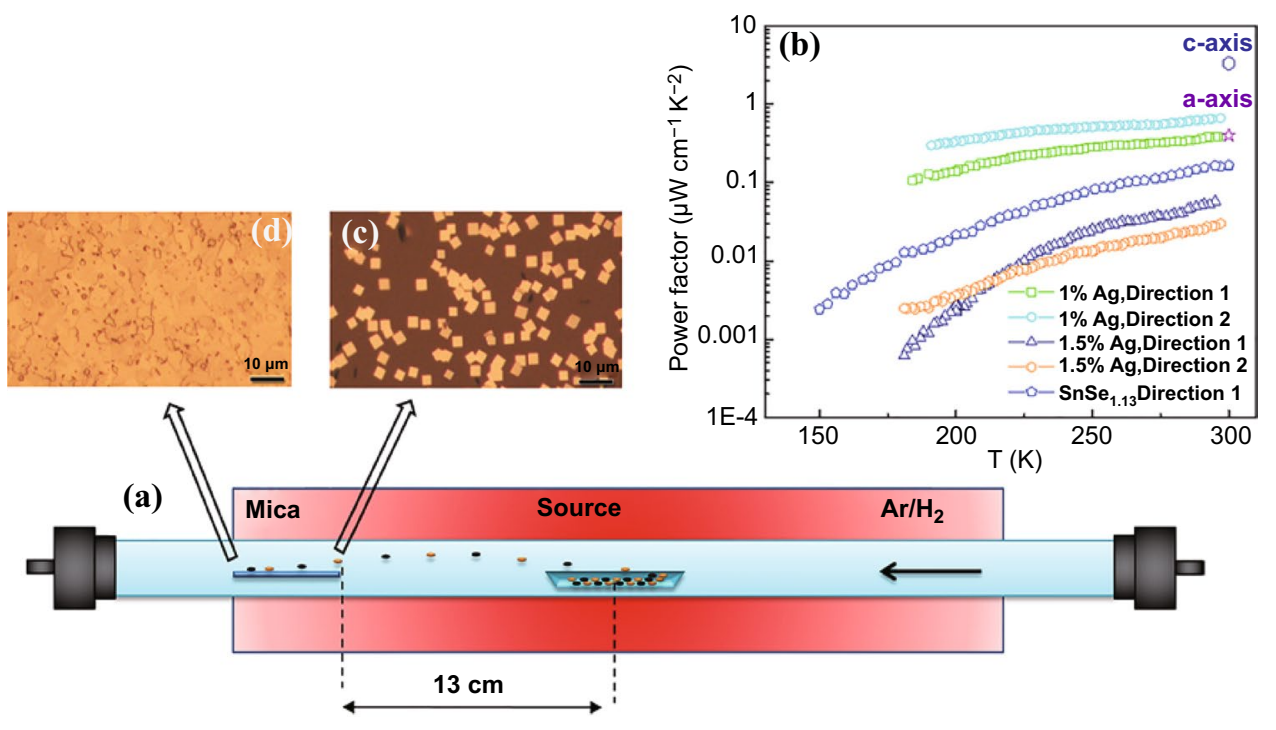

Fig. 12 a SnSe structure and CVD growth. b $P F$ of SnSe and Ag doped SnSe nanoflakes. c SnSe nanoflakes grown in the high-temperature area on substrate. $\mathbf{d}$ nanostructured thin films formed in the low temperature area on substrate. Adapted with permission from Ref. [177] 
polycrystalline films with an axial preferred orientation were obtained. However, with the increase of the temperature, the loss of $\mathrm{Mg}$ element in the thin film made the film towards Sn element rich, which could tune the electronic properties of the $\mathrm{Mg}_{2} \mathrm{Sn}$ thin film. Many other types of semiconducting materials have also been widely studied, including PbTe (111) thin films [184], $\mathrm{Bi}_{2} \mathrm{Se}_{3} / \mathrm{In}_{2} \mathrm{Se}_{3}$ superlattices [185-187]. However, the preparation of 2D semiconducting materials using the MBE approach for TE applications is limited. Cecchi et al. obtained epitaxial $\mathrm{Sb}_{2+x} \mathrm{Te}_{3}$ alloys using MBE to obtain the highest $\sigma, S$, and $P F$ at $1810 \mathrm{~S}$ $\mathrm{cm}^{-1}, 118 \mu \mathrm{V} \mathrm{K}^{-1}$ and $2.52 \mathrm{~mW} \mathrm{~m}^{-1} \mathrm{~K}^{-2}$, respectively. $\mathrm{Hu}$ et al. reported a nanoporous (001)-oriented $\mathrm{Bi}_{2} \mathrm{Te}_{3}$ nanoplate film made by MBE. In their experiment, pure $\mathrm{Bi}$ and $\mathrm{Te}$ element were placed in $\mathrm{SiO}_{2} / \mathrm{Si}$ substrates with an oxidized layer of $600 \mathrm{~nm}$. Upon controlling temperature, annealing and hydrothermal process, nanoporous $\mathrm{Bi}_{2} \mathrm{Te}_{3}$ nanoplates was obtained. HRTEM was used to identify the nanopores in the nanoplates. It was observed that the nanoplates film was changed from intersected to tiling, weakening the carrier scattering along the plane and the carrier mobility. The increased carrier mobility thus could increase the $\sigma$. The carrier concentration of nanoplate films varied with the annealing time also. When the carrier concentration dropped to about $10^{20} \mathrm{~cm}^{-3}$, the $S$ increased to $187.3 \mathrm{mV} \mathrm{K}^{-1}$. This trade-off phenomenon was commonly observed between the $\sigma$ and the $S$. In addition, ethylene glycol was used to treat different $\mathrm{Bi}_{2} \mathrm{Te}_{3}$ nanoplates and it was observed that the boundary density of the pores is the dominant factor to affect the TE performance rather than the total area of the pores because of the boundary density affecting the carrier scattering.

\subsubsection{Wet Chemistry Method}

CVD and MBE could be used to synthesize various semiconducting nanostructure, but some limitations still remain, such as high energy consumption and difficult to control the size of nanostructures. Compared to CVD and MBE, the solution-based chemical synthesis possesses great gains, such as low cost, low energy consumption and easy scalingup. More importantly, chemical synthesis is able to control size, morphology, composition of the 2D materials more easily by regulating delicate reaction parameters such as temperature, concentration catalyst and dispersants. In this section, the chemical synthesis including hydrothermal, solvothermal and solution chemical synthesis for the TE materials will be summarized in details.

3.3.3.1 Hydrothermal Method Aqueous solution-based hydrothermal synthesis has been widely used for the synthesis of 2D type transition metal dichalcogenides [188-190]. Hydrothermal synthesis of 2D materials can provide different structure and morphology, affording a new pathway to change the carrier mobility and concentration, making the synthesized 2D materials as a good candidate for TE applications. Recently, Chen et al. reviewed the hydrothermal method for preparation of various SnSe structures with different dopants for TE studies [191]. Biswas et al. reported 2D nanoplates of Ge-doped SnSe synthesized by the hydrothermal approach together with the SPS process [192]. $\mathrm{SnCl}_{2} \cdot 2 \mathrm{H}_{2} \mathrm{O}$ and $\mathrm{GeI}_{4}, \mathrm{NaOH}$ and $\mathrm{Se}$ powder were placed in a Teflon-lined stainless steel autoclave at $130{ }^{\circ} \mathrm{C}$ for $36 \mathrm{~h}$ to afford the $\mathrm{Sn}_{1-x} \mathrm{Ge}_{x} \mathrm{Se}(\mathrm{x}=1-3 \mathrm{~mol} \%)$ nanoplates (Fig. 13a, b) with a very high yield. Unlike CVD and MBE, this simple synthesis method could be potentially scalable. The as-prepared sample was then densified by the SPS process at $450{ }^{\circ} \mathrm{C}$ to afford the sample for TE performance evaluation. The Ge was doped to the SnSe 2D structure to enhance the carrier concentration in the crystalline structure. TEM revealed that the range of lateral dimension of SnSe nanoplates was within 0.5 to $1.0 \mu \mathrm{m}$. HRTEM also found that lattice spacing between two apparent planes was estimated to be $3.07 \AA$, indicating a set of planes (011) favorable in the structure. The TE performance of the synthesized 2D SnSe nanoplates was evaluated from both directions of II (parallel) and $\perp$ (perpendicular), and their $\sigma$ and $\kappa_{\mathrm{L}}$ are summarized in Fig. 13c-f. Figure 13c, d show that both parallel and perpendicular directions possess the similar $\sigma$ and the trend is also similar starting from semiconducting type to metallic type. The introduction of Ge element enhanced the carrier concentration which significantly improved the $\sigma$. The $S$ was also evaluated for both parallel and perpendicular directions. The $S$ increased in positive correlation with temperature, and reached to a maximum value from 550 to $650 \mathrm{~K}$, consistent with solution-processed SnSe samples. A highest $P F$ was obtained at $\sim 5.10 \mathrm{~mW} \mathrm{~cm}{ }^{-1} \mathrm{~K}^{-2}$ at $3 \mathrm{~mol} \%$ $\mathrm{Ge}$ in $\mathrm{SnSe}$ at $873 \mathrm{~K}$. It was observed that synergistic interaction of lattice anharmonicity, point defects, nanoscale grains, and precipitates reduced the $\kappa_{\mathrm{L}}$ (Fig. 13e, f) in both parallel and perpendicular directions, which assisted in the enhancement of $Z T$ up to $\sim 2.1$ at $873 \mathrm{~K}$.

Zhang et al. reported a facile and rapid synthesis method of $\mathrm{Sb} 2 \mathrm{Te} 3$ hexagonal nanoplate using hydrothermal treatment in the absence of organic solvent or additive [193]. 

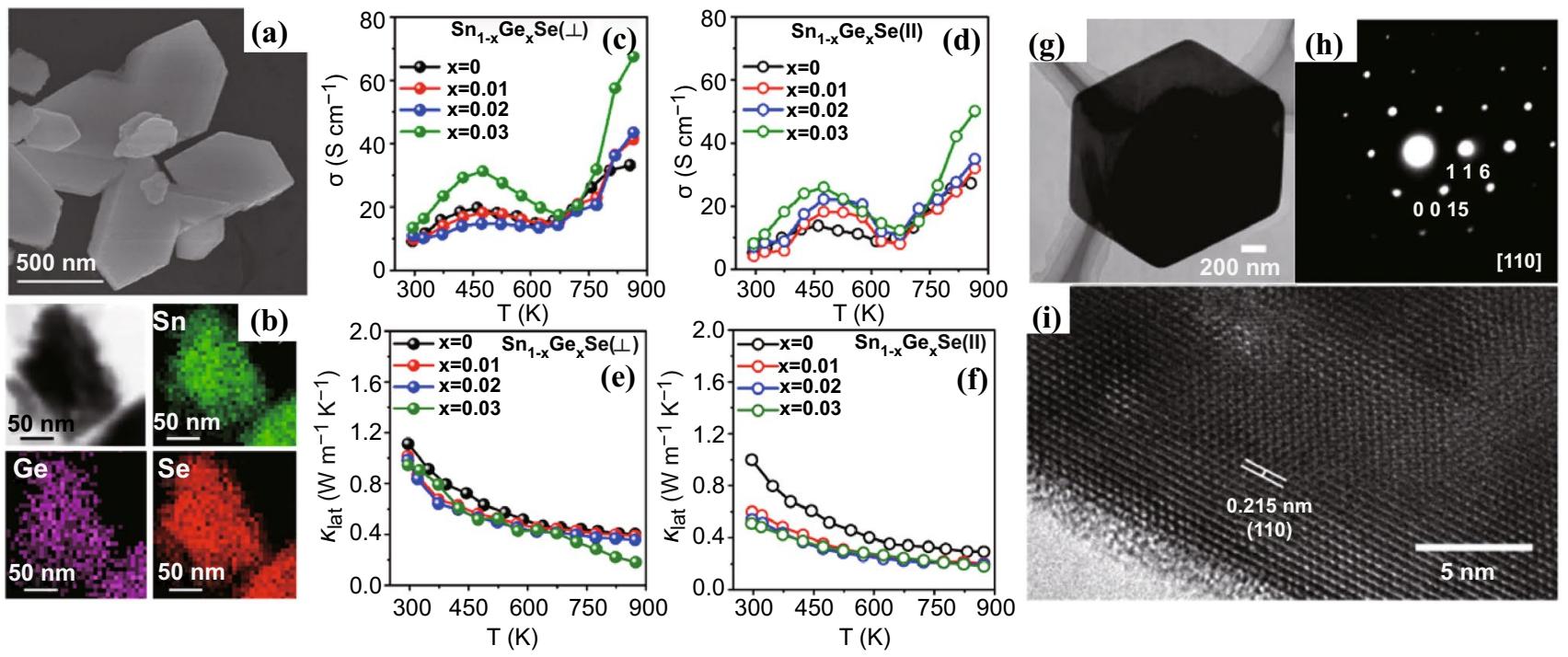

Fig. 13 a FESEM image of $\mathrm{Sn}_{0.97} \mathrm{Ge}_{0.03}$ Se nanoplates. b STEM image of $\mathrm{Sn}_{0.97} \mathrm{Ge}_{0.03} \mathrm{Se}$ nanoplates along with EDAX color mapping for Sn, Ge, and Se. Temperature-dependent $\mathbf{c}, \mathbf{d}$ electrical conductivity and $\mathbf{e}, \mathbf{f}$ lattice thermal conductivity of $\mathrm{Sn}_{1-x} \mathrm{Ge}_{x} \mathrm{Se}$ measured parallel (open symbols) and perpendicular (solid symbols) to the SPS pressing direction, respectively. $\mathbf{g}$ TEM image, $\mathbf{h}$ SAED pattern and $\mathbf{i}$ HRTEM image of a single $\mathrm{Sb}_{2} \mathrm{Te}_{3}$ nanoplate. Adapted with permission from Refs. [192, 193]

In the synthesis of this nanoplate, $\mathrm{SbCl} 3$ and tartaric acid, $\mathrm{NH} 3 \cdot \mathrm{H} 2 \mathrm{O}$ solution, $\mathrm{K} 2 \mathrm{TeO} 3$, and $\mathrm{N} 2 \mathrm{H} 4 \cdot \mathrm{H} 2 \mathrm{O}$ were placed in an autoclave, and the reaction temperature was controlled at $180^{\circ} \mathrm{C}$ for $5 \mathrm{~h}$, followed by filtration to obtain the $\mathrm{Sb} 2 \mathrm{Te} 3$ nanoplates. Figure $13 \mathrm{~g}$ shows a HRTEM image of a typical single $\mathrm{Sb}_{2} \mathrm{Te}_{3}$ hexagonal nanoplate. As seen in Fig. 13h, the Sb2Te3 nanoplate is a well-crystallized single crystal. Figure 13i displays a well-resolved 2D lattice fringe with a plane spacing of $0.22 \mathrm{~nm}$, in accordance with the lattice planes of (110) in rhombohedral $\mathrm{Sb} 2 \mathrm{Te} 3$ nanoplates. The hydrothermal reaction conditions to obtain the $\mathrm{Sb}_{2} \mathrm{Te}_{3}$ hexagonal nanoplate are very critical and it requires to be wellcontrolled to avoid the formation of nanoparticle or NW. Using the home-made setup, the $S$ of $\mathrm{Sb}_{2} \mathrm{Te}_{3}$ nanoplate was evaluated to be $125 \mathrm{mV} \mathrm{K}^{-1}$ as p-type semiconductor using a home-made setup, which is higher than these of other type nanocrystals, such as $\mathrm{Sb}_{2} \mathrm{Te}_{3}$ nanoparticles and nanorods. Due to the anti-site effect derived from $\mathrm{Sb}$ atom on the $\mathrm{Te}$ lattice sites, the $S$ is approximately 1.6 times of that value of undoped bulk crystals at $79 \mathrm{mV} \mathrm{K}^{-1}$, which is attributed to the energy filtering due to the nano-boundaries created by the extra atom throughout the doping process.

3.3.3.2 Solvothermal Method As one of the best TE materials, antimony telluride ( $\mathrm{Sb} 2 \mathrm{Te} 3$ ) has been well investigated as a p-type semiconductor [30,194]. However, the study of how the shape and size changes in 2D type $\mathrm{Sb} 2 \mathrm{Te} 3$ nanomaterials is limited. Lee et al. reported a solvothermal approach to prepare the $\mathrm{Sb} 2 \mathrm{Te} 3$ nanoplates. $\mathrm{SbCl} 3, \mathrm{~K} 2 \mathrm{TeO} 3$ and $\mathrm{PVP}$ were dissolved in diethylene glycol, followed by the addition $\mathrm{NaOH}$ aqueous solution. Upon the solvothermal process in a Teflon-lined stainless-steel autoclave at $230{ }^{\circ} \mathrm{C}$ for $24 \mathrm{~h}$, the corresponding nanoplates were obtained. SEM analysis showed the edge lengths and thicknesses of the nanoplates are 3-5 $\mu \mathrm{m}$ and $\sim 100 \mathrm{~nm}$ respectively. Sequentially, the TE performance was evaluated in comparison with the sample prepared by the ball milling. Interestingly, the $\kappa$ of the $\mathrm{Sb} 2 \mathrm{Te} 3$ nanoplates is much lower than that of samples obtained from the ball milling process. Such a low $\kappa$ could be attributed to the grain boundary generated between nanoplates. The $S$ is in the range of $300-350 \mu \mathrm{V} \mathrm{K}^{-1}$, about two times higher than those of $\mathrm{Sb} 2 \mathrm{Te} 3$ prepared by the melting method $\left(102-144 \mu \mathrm{V} \mathrm{K}^{-1}\right)$. The improvement in the $S$ can arise from the energy filtering effects caused by the boundaries between $\mathrm{Sb} 2 \mathrm{Te} 3$ nanoplates.

As another important class of semiconducting materials, $2 \mathrm{D} \mathrm{Bi}_{2} \mathrm{Te}_{3}$ nanostructures have attracted great attention to enhance the TE performance. Takashiri et al. used $\mathrm{Bi}_{2} \mathrm{O}_{3}$ and $\mathrm{TeO}_{2}$ under basic conditions via a solvothermal process to obtain the $\mathrm{Bi}_{2} \mathrm{Te}_{3}$ nanoplate, followed by electrical deposition and annealing to afford the electrodeposited layers [195, 196]. Annealing at $250{ }^{\circ} \mathrm{C}$ helped reduce the boundaries 
between nanoplates, and further enhanced the $\sigma$ and $S$ by about $200 \%$ and $50 \%$, respectively. Thermal annealing improved the crystallinity of electrodeposited layers, which decreased the number of defects (carrier concentrations) and the number of boundaries (increasing mobility). The structure variation arising from the post treatment could significantly improve the TE performance through tuning the carrier concentration and mobility. Chen et al. reported a solvothermal method to introduce high porosity in $\mathrm{Bi}_{2} \mathrm{Te}_{3}$ hexagonal plates and decrease the overall $\kappa$ as well as the $\kappa_{\mathrm{L}}$ [197]. Through reduction of the $\kappa$, the TE performance could be enhanced significantly when the $P F$ remained unchanged. In the synthesis of $\mathrm{Bi}_{2} \mathrm{Te}_{3}$ nanoplates (Fig. 14a), $\mathrm{Bi}_{2} \mathrm{O}_{3}$ and $\mathrm{TeO}_{2}$ were placed in an autoclave in the presence of $\mathrm{NaOH}$ and ethylene glycol at $210{ }^{\circ} \mathrm{C}$ for $24 \mathrm{~h}$. The $\mathrm{Bi}_{2} \mathrm{Te}_{3}$ nanoplates were obtained through filtration. Then the obtained $\mathrm{Bi}_{2} \mathrm{Te}_{3}$ nanoplates were subjected to the SPS process and sublimation to obtain the sample for the structure characterization. SEM analysis showed the $\mathrm{Bi}_{2} \mathrm{Te}_{3}$ nanoplate has the thickness of $20 \mathrm{~nm}$ (Fig. 14b, c) and can induce a high density of grain boundaries in the pellet after the sintering. As shown in Fig. 14d, the sintering process could introduce the density of pores in the structure and the pore size is at a size of $\sim 400 \mathrm{~nm}$ in the matrix. Sequentially, the $\sigma$ and
$S$ were measured in comparison with the dense sample. It was found that the similar value was obtained between the dense sample in other literature and the as-prepared porous structure, leading to a similar magnitude of the $P F$. The porous structure in the matrix could significantly reduce the $\kappa_{\mathrm{L}}$ to less than $0.1 \mathrm{~W} \mathrm{~m}^{-1} \mathrm{~K}^{-1}$ (Fig. 14e) due to the phonon gas theory. Owing to the overall reduction in the $\kappa$, the $Z T$ value was improved dramatically up to 0.97 at $420 \mathrm{~K}$ (Fig. 14f), the highest values reported for pure n-type $\mathrm{Bi}_{2} \mathrm{Te}_{3}$ semiconductors.

Zou et al. reported a microwave-assisted solvothermal synthesis method to obtain $\mathrm{Bi}_{2} \mathrm{Te}_{3-x} \mathrm{Se}_{x}$ nanoplates which could enhance the TE performance [198]. In the synthesis of $\mathrm{Bi}_{2} \mathrm{Te}_{3-x} \mathrm{Se}_{x}$ nanoplates, $\mathrm{Bi}\left(\mathrm{NO}_{3}\right)_{3} \cdot 5 \mathrm{H}_{2} \mathrm{O}, \mathrm{Na}_{2} \mathrm{TeO}_{3}$ and $\mathrm{Na}_{2} \mathrm{SeO}_{3}$ were placed in reaction vessel in the presence of ethylene glycol and $\mathrm{NaOH}$ at $230{ }^{\circ} \mathrm{C}$ for 5 min. During the synthesis, various loadings of Se were doped in the samples to substitute the Te element to study its impact on the TE performance. The obtained $\mathrm{Bi}_{2} \mathrm{Te}_{3-x} \mathrm{Se}_{x}$ nanoplates were subjected to the SPS process to make the pellets for the TE measurement. It was found that the loading of Se element doesn't seem to have huge impact on the overall performance, leading to the $P F$ in the range of $1.5-1.9 \mathrm{~mW}$ $\mathrm{m}^{-1} \mathrm{~K}^{-2}$. The texture fraction in the nanostructure materials

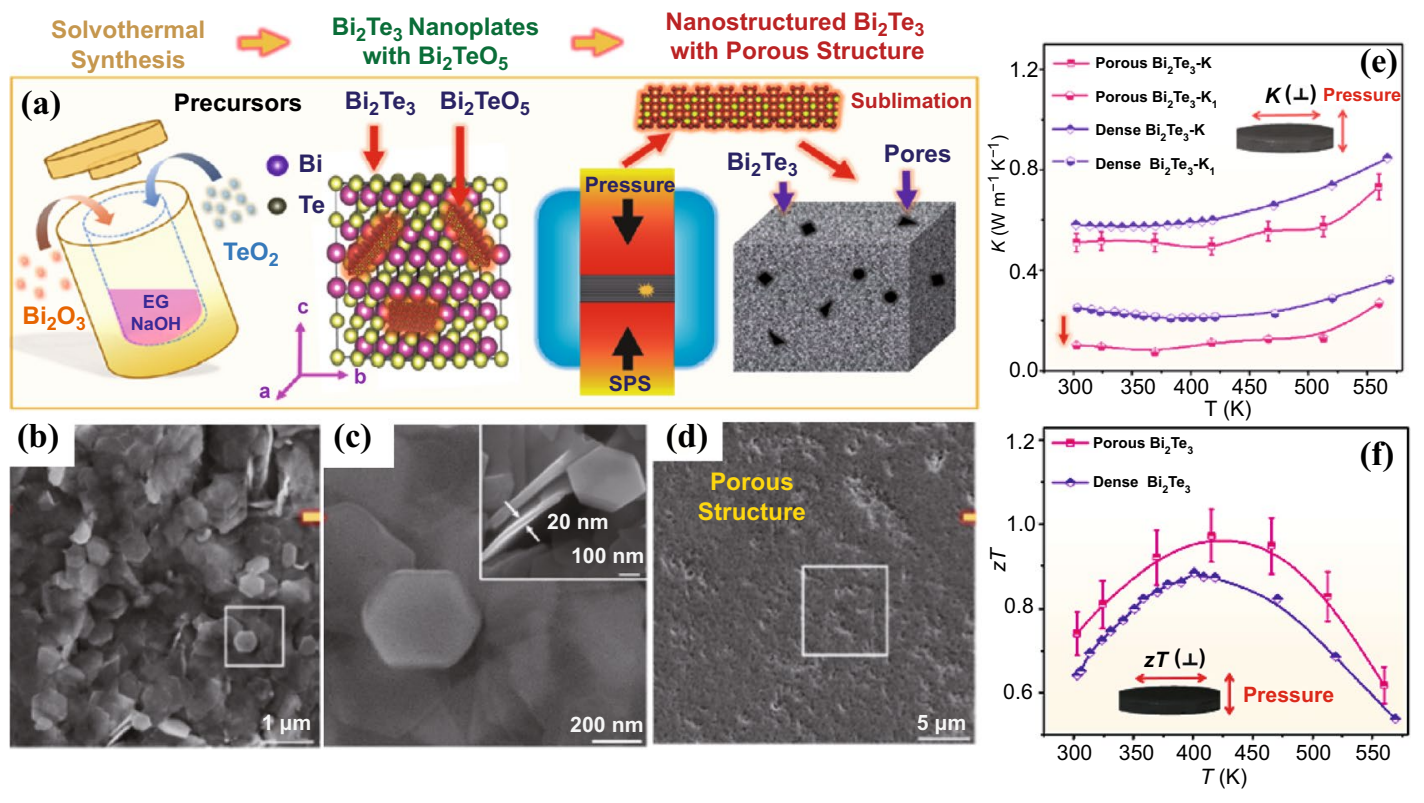

Fig. 14 a Illustration of the solvothermal synthesis of $\mathrm{Bi}_{2} \mathrm{Te}_{3}$ nanoplates followed by SPS to fabricate a porous nanostructured $\mathrm{Bi}_{2} \mathrm{Te}_{3}$ pellet. SEM images of $\mathbf{b}, \mathbf{c}$ the as-synthesized $\mathrm{Bi}_{2} \mathrm{Te}_{3}$ nanoplates and $\mathbf{d}$ the as-sintered nanostructured porous $\mathrm{Bi}_{2} \mathrm{Te}_{3}$. Temperature dependence of $\mathbf{e} \kappa$ and $\kappa_{\mathrm{L}}, \mathbf{f} \mathrm{ZT}$ of the as-sintered nanostructured porous $\mathrm{Bi}_{2} \mathrm{Te}_{3}$ pellet compared with dense nanostructured $\mathrm{Bi}_{2} \mathrm{Te}_{3}$. Adapted with permission from Ref. [197] 
is smaller than that of the bulk polycrystalline counterpart, and can help reduce the phonon scattering, leading to the reduction in the $\kappa$. A lowest $\kappa$ of $0.69 \mathrm{~W} \mathrm{~m}^{-1} \mathrm{~K}^{-1}$ value was achieved in the $\mathrm{Bi}_{2} \mathrm{Te}_{2.7} \mathrm{Se}_{0.3}$ pellet, which is much lower than the value between 1.5 and $2 \mathrm{~W} \mathrm{~m}^{-1} \mathrm{~K}^{-1}$ of their bulk counterpart materials. Such reduction is attributed to the nanostructure grains and boundaries in the pellet made by the $\mathrm{Bi}_{2} \mathrm{Te}_{3-x} \mathrm{Se}_{x}$ nanoplates. There are mainly two factors promoting the reduction of the $\kappa$ namely the Umklapp phonon-phonon scattering by the inherently strong anharmonicity and the wide frequency phonon scatterings caused by the multi-scattering pathways. The same group also performed the theoretical studies on the thermal transport and phonon transport to understand the rationale behind the $\kappa$ reduction, revealing that the complex carrier scatterings helped suppress the bipolar effect and weakened the dependence of transport properties on the carrier movement.

3.3.3.3 Solution-Phase Synthesis Hydrothermal and solvothermal approaches have been used to grow 2D type inorganic semiconducting materials. These two methods usually require the reaction to be performed at high temperature (e.g., from 150 to $300{ }^{\circ} \mathrm{C}$ ) in a sealed stainless vessel. At this reaction condition, the inorganic species in general can grow in a specific direction to form the 2D type of materials. As another approach, solution chemical synthesis can be used to prepare $2 \mathrm{D}$ semiconducting materials with a lot of benefits, including tunable reaction conditions and easy scale-up. Cabot and co-workers reported a chemical synthesis method using tin selenide molecular precursor under solution process to prepare 2D SnSe nanoplate for TE studies [199]. In the synthesis of 2D SnSe nanoplate, tin chloride selenium dioxide, tri-n-octylphosphine and oleyamine were premixed in a very short of time, followed by the addition of oleic acid to produce dentritic SnSe nanostructures. Then the SnSe precursor was injected into a reaction flask preheated at $420{ }^{\circ} \mathrm{C}$ for decomposition to prepare the SnSe nanostructures. TEM and SEM characterization of SnSe nanostructure showed that as-prepared nanoplates had the size of $4 \pm 1 \mu \mathrm{m}$ and a thickness of $90 \pm 20 \mathrm{~nm}$ and the crystal followed the [100] crystal direction oriented along the pressure axis by XRD analysis. TE property study revealed that both cross-plane and in-plane afforded very similar $\sigma$ of $15-30 \mathrm{~S} \mathrm{~cm}^{-1}$. A slightly higher $S$ of $400 \mu \mathrm{V} \mathrm{K}^{-1}$ was obtained in the cross-plane direction, which could be attributed to the energy filtering introduced by the grain boundaries, specifically the preferential scattering of the low-energy carriers at the plate interfaces. A very low $\kappa$ was obtained in both directions, significantly improving the $Z T$ value of the $\mathrm{SnSe}$ nanoplates. The low $\kappa$ could be arisen from the low $\kappa_{\mathrm{L}}$ because of the phonon scattering.

Biswas et al. reported a chemical synthesis to prepare several n-type ultrathin layers of Bi doped $\mathrm{SnSe}$ nanosheets [200, 201]. In the synthesis of SnSe nanosheets, $\mathrm{SnCl}_{4} \cdot 5 \mathrm{H}_{2} \mathrm{O}$ and $\mathrm{SeO}_{2}$ were taken in a mixture of oleylamine and 1,10-phenanthroline. The reaction was performed at $200{ }^{\circ} \mathrm{C}$ under $\mathrm{N}_{2}$ followed by filtration to afford the nanostructured materials at the gram scale. Bi precursor was used in the reaction to dope the nanosheet to afford $\mathrm{Sn}_{0.94} \mathrm{Bi}_{0.06} \mathrm{Se}$. Powder X-ray diffraction analysis shows the $2 \mathrm{D}$ SbSe nanosheet has the lattice parameters of $\mathrm{a}=11.50 \AA, \mathrm{b}=4.15 \AA$, and $\mathrm{c}=4.45 \AA$ and the indirect band gap was measured to be $\sim 0.88 \mathrm{eV}$. Field emission scanning electron microscope (FESEM) showed that the Bi doped $\mathrm{SnSe}$ nanosheets possessed a thickness of $1.2-3 \mathrm{~nm}$. Such a dimension is critical to create the nanoscale grain boundaries and point defects across the interphase between Se-Se layers. Such boundaries are effective for the phonon scattering and improvement of TE performance. Based on the Hall Effect measurement, the introduction of Bi element in the SnSe could significantly enhance the carrier concentration, leading the $\sigma$ up to $12.5 \mathrm{~S} \mathrm{~cm}^{-1}$ at $750{ }^{\circ} \mathrm{C}$ for the cross-plane direction. Negative $S$ was observed for both cross- and in-plane directions. The highest $P F$ of $100 \mu \mathrm{W} \mathrm{m}{ }^{-1} \mathrm{~K}^{-2}$ was obtained for $\mathrm{Sn}_{0.94} \mathrm{Bi}_{0.06} \mathrm{Se}$ nanosheets, much greater than that of SnSe. More interestingly, the nanoboundaries in the nanosheet could facilitate the phonon scattering, leading to a low $\kappa_{\mathrm{L}}$ of $\sim 0.3 \mathrm{~W} \mathrm{~m}^{-1} \mathrm{~K}^{-1}$. This low $\kappa$ could contribute significantly to the $Z T$ enhancement to 0.21 at about $700{ }^{\circ} \mathrm{C}$ for $\mathrm{Bi}$ doped SnSe nanosheets. Similarly, $\mathrm{Hu}$ et al. reported a chemical synthesis method using $\mathrm{SnCl}_{2} \cdot 2 \mathrm{H}_{2} \mathrm{O}$ and $\mathrm{Se}$ in the basic solution to prepare $\mathrm{SnSe} 2 \mathrm{D}$ nanosheets [202]. In their work, extremely low $\kappa$ of $0.09 \mathrm{~W} \mathrm{~m}^{-1} \mathrm{~K}^{-1}$ was obtained, demonstrating the thin film made from $2 \mathrm{D}$ nanosheet could be used for low-grade waste heat recovery.

Zou et al. reported a chemical method to dope Te element into the SnSe nanosheets to enhance the TE performance [203]. In the synthesis of $\mathrm{SnSe}$ nanosheets, $\mathrm{SnCl}_{2} \cdot 2 \mathrm{H}_{2} \mathrm{O}, \mathrm{Na}_{2} \mathrm{SeO}_{3}$, and $\mathrm{Na}_{2} \mathrm{TeO}_{3}$ were added into the $\mathrm{NaOH}$ solution in ethylene glycol. After the stirring, the reaction was heated at $230{ }^{\circ} \mathrm{C}$ for $10 \mathrm{~min}$ to obtain the Te doped SeSe nanoplates. SEM and TEM analysis shows the nanoplates have the average lateral size and thickness of 
about $10 \mu \mathrm{m}$ and $200 \mathrm{~nm}$, respectively. With the increasing Te element in the SnSe nanoplates, the $S$ dropped from 365 to about $341 \mu \mathrm{V} \mathrm{K}^{-1}$. Due to the trade-off relationship between the $\sigma$ and $S$. The $\sigma$ improved doubly from 50 to about $100 \mathrm{~S} \mathrm{~cm}^{-1}$. The Te element in the alloy could reduce the band gap and the hole concentration, resulting in a stronger bipolar effect, and thus increase in the $\sigma$ and reduction in the $S$.

Another noteworthy point is that with increasing Te element into the alloy, the point defects were introduced, which enhanced the phonon scattering and led the $\kappa$ to be reduced by about $30 \%$ overall. In this case, the average $Z T$ of 0.58 was obtained. Gregory et al. reported a similar method to synthesize SnSe nanoplates using water as solvent without using any surfactant [204]. The $P F$ is eightfold higher than that of material made using citric acid as a structuredirecting agent. As another type of important semiconducting materials, $2 \mathrm{D} \mathrm{Bi}_{2} \mathrm{Te}_{3}$ nanostructure is also well-studied, especially in 1D type. Hyeon et al. reported a chemical method to prepare n-type of ultrathin $\mathrm{Bi}_{2} \mathrm{Te}_{3}$ nanoplates with the improved TE performance [205]. In the synthesis, bismuth neodecanoate and tri-n-octylphosphine-tellurium were used as $\mathrm{Bi}$ and $\mathrm{Te}$ sources in polyamine, respectively. 1-Dodecanethiol was used with the proper concentration to form the $\mathrm{Bi}_{2} \mathrm{Te}_{3}$ nanoplates. The thickness of the nanoplates was about $1 \mathrm{~nm}$. Sequentially the as-prepared nanoplates were sintered into pellets for the TE performance measurement. The $S$ was in the range of -130 to $-160 \mu \mathrm{V} \mathrm{K}^{-1}$. It was noteworthy that the electron concentrations and mobility varied at different sintering temperatures possibly because of the interphase effect, which facilitated the carrier scattering and defects by the organic residue from the synthesis. Meantime, the $\kappa$ was observed to be reduced significantly (lowest at about $0.4 \mathrm{~W} \mathrm{~m}^{-1} \mathrm{~K}^{-1}$ ). This phenomenon was also observed in other $2 \mathrm{D}$ materials and this can be attributed to the scattering of carriers and boundaries between the 2D materials.

\subsection{D Nanostructure}

One simple approach to improve TE property in bulk materials is to induce the formation of porosity. Although the traditional approach to TE materials was to create bulk samples with very little remaining porosity ( $>99 \%$ relative density), many recent studies have shown that either random or template-assisted uniform porosity can be beneficial for TE performance when properly optimized [21, 206]. Reported studies have shown that the sizes of the pores in TE materials and their distribution pattern play critical role in TE properties. To maintain sufficient $\sigma$, these structures generally must be sintered to at least $80 \%$ theoretical density, effectively limiting the porosity.

\subsubsection{Random Porous Structure}

Randomly porous structures are created by deliberately partially densifying a material to generate a distribution of pores throughout the structure. Randomly porous structures are easily synthesized but the effects of nano-structuring on the $\kappa$ (and in some cases, the $S$ ) are much weaker due to the lack of morphological control.

In 2018, Xu et al. synthesized PbS nanocrystals with different shapes and consolidated them into highly porous and well crystalline monoliths using the SPS process (Fig. 15a) [207]. It was found the relative density and TE performance of the porous $\mathrm{PbS}$ monoliths could be tuned simultaneously (Fig. 15b, c). The as-obtained porous monolith with a large grain size has low relative mass density $(82 \%)$ and high porosity, and also exhibits a high $\sigma$, a low $\kappa$, and hence an excellent $Z T(1.06$ at $838 \mathrm{~K})$. The origin of high $Z T$ was studied by DFT calculations, indicating that high $Z T$ can be attributed to enhanced scattering of phonons caused by the porous structures.

In 2019, Shi et al. employed a 3D printing technique (Fig. 15d) to fabricate porous bismuth antimony telluride $\left(\mathrm{Bi}_{0.5} \mathrm{Sb}_{1.5} \mathrm{Te}_{3}, \mathrm{BST}\right)$ and its TE properties were studied [208]. The porous TE samples were fabricated with the selective laser sintering method using 100 mesh BST powder. Energy density of the laser light that scans across the thin layer of TE powder was carefully controlled to ensure that TE powder was only partially melted to form the desired porous TE material. The laser sintering process of BST particles led to the formation of many micrometer- and nanometer-sized random pores, accounting for the reduction of the $\kappa$. The minimum $\kappa$ of the porous BST samples was measured to be $0.27 \mathrm{~W} \mathrm{~m}^{-1} \mathrm{~K}^{-1}$ at $54{ }^{\circ} \mathrm{C}$. The reduction in the $\kappa$ is attributed to the boundaries and defects formed during the selective laser sintering process as well as the porous structures 
(a) Before Sintering After Sintering

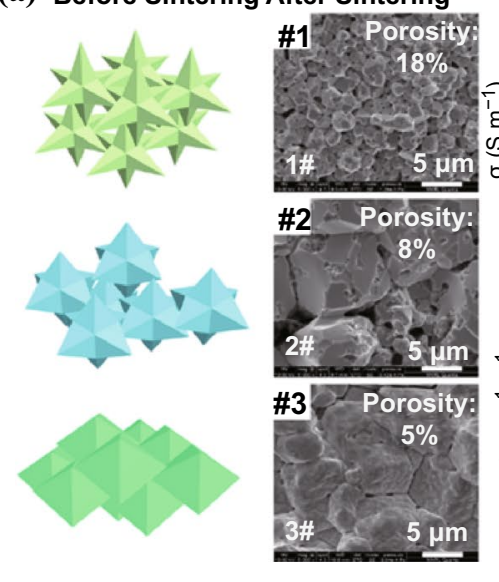

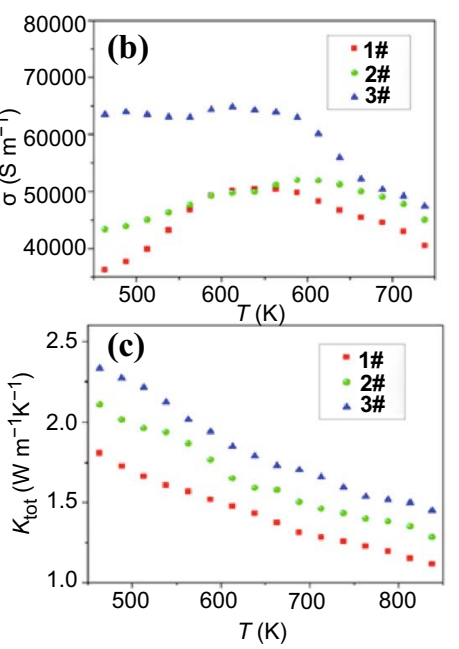

(d)

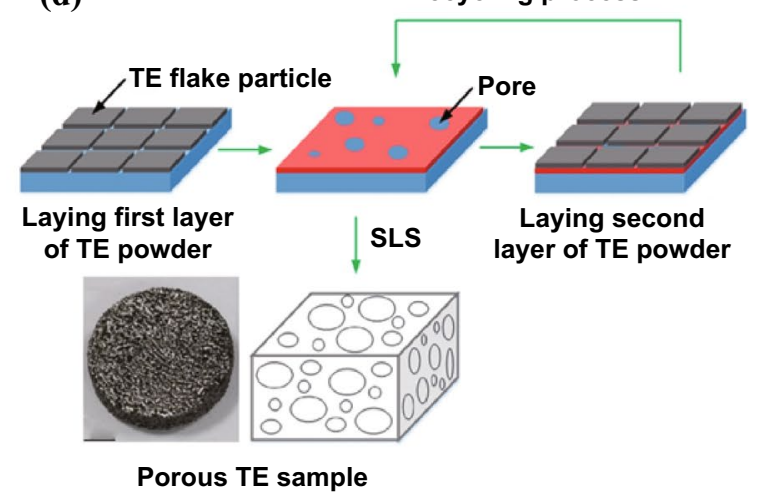

Fig. 15 a Schematic illustration (left) and SEM images (right) of the as-sintered porous monoliths from 1\# (hexapods), 2\# (less-protruding hexapods) and 3\# (octahedra) PbS nanocrystals. Temperature dependence of $\mathbf{b} \sigma$ and $\mathbf{c} \kappa$ of the three sintered TE monoliths. d Schematic diagram of selective laser sintering 3D printing process to prepare porous TE monoliths. Adapted with permission from Refs. [207, 208]

of the materials. The $Z T$ of the porous samples was found to have a maximum value of about 1.29 at $327 \mathrm{~K}$, higher than that of the BST bulk material.

\subsubsection{Template-Assisted Uniform Porous Structure}

In 2017, Hong et al. reported the preparation of a periodic 3D nanostructured TE monolith, with an approximate pore size of $140 \mathrm{~nm}$, by electrochemical deposition of a Bi-Sb-Te ternary alloy into a highly ordered, interstitial porous network in an epoxy template predefined by advanced lithography (Fig. 16a) [209]. The electroplating conditions for the 3D nanoconfined geometry was optimized to facilitated the uniform and dense filling of $\mathrm{Bi}_{1.5} \mathrm{Sb}_{0.5} \mathrm{Te}_{3}$ into the template over a large area of $625 \mathrm{~mm}^{2}$. Most nanostructured TE materials suffer from undesirable degradation of the $\sigma$. In contrast, the 3D nanostructures, however, are able to maintain electron transport properties because the sizes of the TE struts in a structural unit cell are sufficiently larger $(140 \mathrm{~nm})$ than the mean free path of electrons ( 40 to $60 \mathrm{~nm})$. It is suggested that the extrinsic phonon scattering at the interfaces of the nanostructures without changing electrical transport is responsible for the selective reduction of the $\kappa$ while the $S$ and $\sigma$ are almost intact (Fig. 16b). The 3D nanostructure successfully resulted in a decreased $\kappa$ from 1.14 to $0.89 \mathrm{~W} \mathrm{~m}^{-1} \mathrm{~K}^{-1}$, at $350 \mathrm{~K}$ while maintaining a $\sigma$ of
644.5 $\mathrm{S} \mathrm{cm}^{-1}$ and a $\kappa$ of $144 \mathrm{mV} \mathrm{K}^{-1}$ (Fig. 16c, d). The 3D nanostructured $\mathrm{Bi}_{1.5} \mathrm{Sb}_{0.5} \mathrm{Te}_{3}$ film showed an improved $Z T$ value of 0.56 at $400 \mathrm{~K}$, which was approximately $50 \%$ higher than the value for an ordinary $\mathrm{Bi}_{1.5} \mathrm{Sb}_{0.5} \mathrm{Te}_{3}$ film. In 2018, the same group also reported the deposition of nanoscaled $\mathrm{ZnO}$ film on the 3D nanostructured epoxy template via the atomic layer deposition [210]. In this work, the suppressed $\kappa$ of the $3 \mathrm{D} \mathrm{ZnO}$ film is $\sim 3.6 \mathrm{~W} \mathrm{~m}^{-1} \mathrm{~K}^{-1}$ at $333 \mathrm{~K}$, which is $\sim 38$ times lower than that of the blanket $\mathrm{ZnO}$ film.

\subsection{Nanoscale Doping in Bottom-up Process}

For semiconductor thermoelectric materials, the introduction of small quantities of impurity (element doping) during the bottom-up preparation can not only tailor the carrier concentration and/or mobility to optimize the $\sigma$, but also induce point defects (vacancies or self-interstitials) and adjust the microstructures (e.g., phase separation, formation of nanoscaled precipitates or ultra-fine grains) to reduce the $\kappa_{\mathrm{L}}$ [211-220]. Although the carrier concentration in a material can be modulated using defect-engineering, or stoichiometry control during synthesis, reaching the optimized carrier concentration (typically $10^{19} \mathrm{~cm}^{-3}$ ) often require additional extrinsic doping. Intuitively, this $10^{19}$ carrier concentration is equivalent to about 1-30 electronically active sites in a $10 \mathrm{~nm}$ particle. Schematic illustrating doping mechanisms 

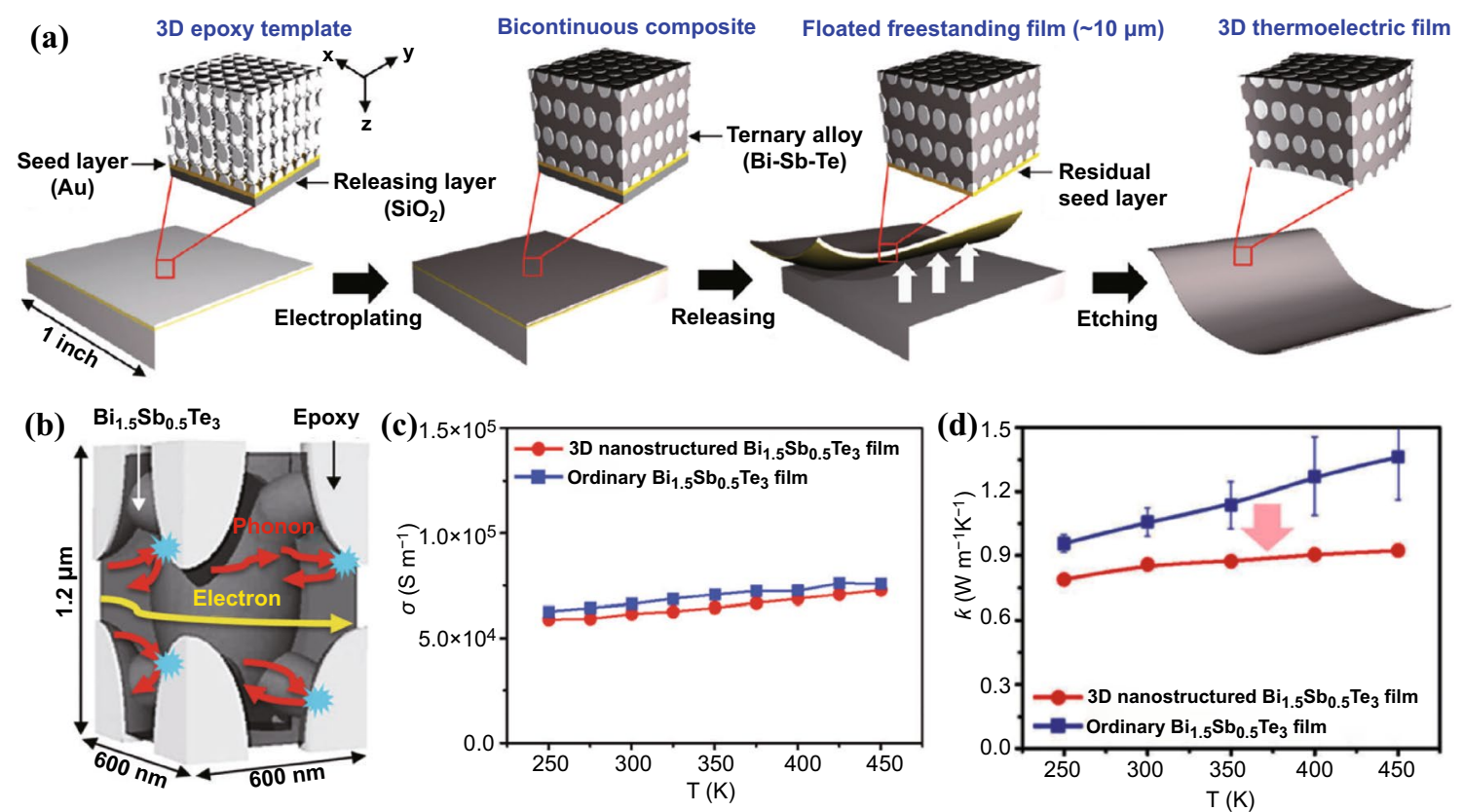

Fig. 16 a Schematic illustration of procedures for the fabrication of a freestanding and transferrable 3D nanostructured TE film. b Structural unit cell of a 3D nanostructured $\mathrm{Bi}_{1.5} \mathrm{Sb}_{0.5} \mathrm{Te}_{3}$ film illustrating the concept of increasing phonon boundary scattering without altering electrical transport. Comparison of $\mathbf{c}$ electrical conductivity and $\mathbf{d}$ thermal conductivity of the $3 \mathrm{D}$ nanostructured and ordinaryBi ${ }_{1.5} \mathrm{Sb}_{0.5} \mathrm{Te}_{3}$ films as a function of temperature. Adapted with permission from Ref. [209]

(a)

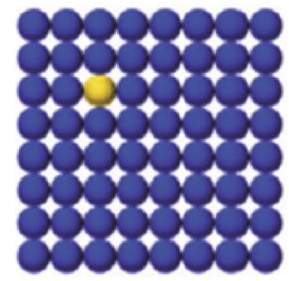

(d)

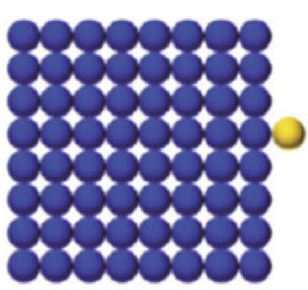

(b)

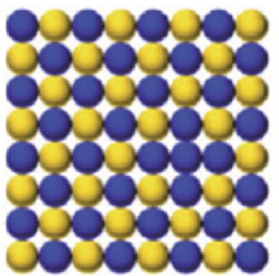

(e)

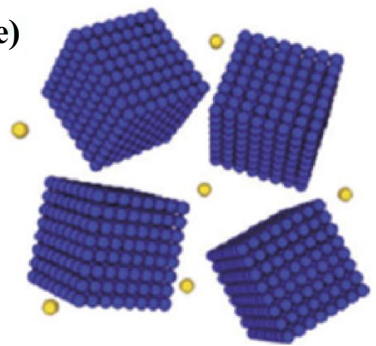

(c)

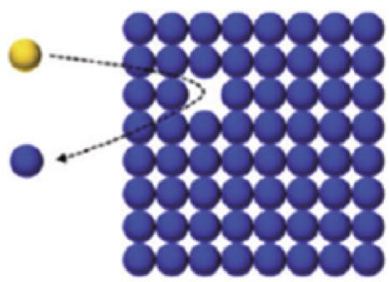

(f)

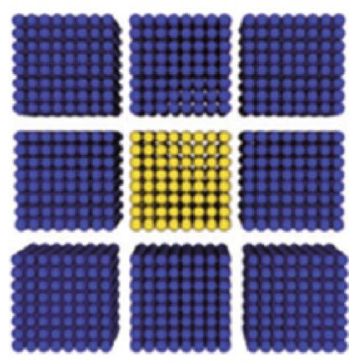

Fig. 17 Scheme of possible strategies to tune doping in nanoparticle-based solution-processed nanomaterials: a extrinsic dopants during synthesis; b stoichiometry control; $\mathbf{c}$ post-synthesis atomic substitution; $\mathbf{d}$ surface chemistry; e dopant additives during consolidation; $\mathbf{f}$ modulation doping. Adapted with permission from Ref. [22]

in nanostructures is shown in Fig. 17. The most commonly used strategy in modulating the carrier concentration at the nanoparticle level is the incorporation of dopants at the initial reaction solution [221]. In this approach, the dopants and host precursors are homogeneously mixed before the nucleation, resulting in uniform doping. However, not all dopants can be incorporated using this method. The coordinating ligands and proper control of reaction conditions are critical to enable high doping efficiency of these extrinsic elements rather than just a secondary phase or 
nanocomposite. In addition, surfactants, which can be used to modulate surface energies, can either inhibit or promote the incorporation of external dopant species. Generally, the addition of any type of impurities will affect the growth kinetics and thermodynamics of these nanostructures. Therefore, understanding the solubility and kinetics of the dopants and the host is very important to control the optimal amount of dopant that can be incorporated into these materials.

Alternatively, dopant ions can be introduced within the preformed nanoparticles. Ion exchange processes can be employed in this case, which is akin to ion-implantation in semiconductor processing. Although this is a more expensive strategy, a non-equilibrium thermodynamic doping process can be achieved, thus making it possible to introduce a large amount of dopant beyond the solubility limit allowed by thermodynamics [222]. Besides introducing impurities, a common strategy to tune carrier concentration in nanoscale level (especially in 2D structures) is by applying gate-voltage [223]. In essence, gate voltage not only provides carrier concentration modulation, but can also be used to alter the carrier scattering mechanism, resulting in multi-fold enhancements of thermoelectric properties by enhancing electrical conductivity and reducing thermal conductivity.

\section{Conclusion and Outlook}

In this review paper, we provided a comprehensive review on the state-of-the-art strategies and bottom-up approaches that had been employed for constructing nanostructured semiconductor TE materials with different dimensions, focusing on the relationships between the structures and the key electronic and thermal transport parameters contributing to $Z T$, such as the $S$ enhancement due to the energy filtering or quantum confinement effects, and the $\kappa$ reduction inherently due to the phonon scattering. Table 1 summarizes the TE properties of various nanostructured materials prepared via bottom-up approaches. The vapor-based processes are expected to play an important role in preparing high-precision materials for theoretical mechanism studies and/or fabrication of micron-scale TE devices, while the solution-based preparation methods with controllable size, morphology and surface chemistry offer a convenient route towards inexpensive and scalable low-dimensional TE materials. However, the practical adoption of those liquid-based processes towards high quality nanomaterials still remains a challenge. Scale-up production of these nanostructured materials inevitably requires automated processes with nearly $100 \%$ yields, high nanoparticle concentrations in solution, low cost and environmentally-benign precursors, solvents, reductants and surfactants, as well as the recycling of solvents and possible side-products. Moreover, the consolidated conditions which may cause significant atomic and interface redistribution should also be investigated and optimized to assemble the nanostructured materials with a maximum retention of their electronic and phononic characteristics. Deep fundamental understanding of the electronic and thermal transport properties of these nanostructured materials will be highly valuable for further development of advanced, practical and high-performance TE devices. In addition, through developments in low-dimensional materials, some promising concepts such as nano-porous structures have been applied and have seen some early successes in traditional 3D bulk materials. Remarkably, it has been shown that it is in fact possible for porous structures to be $3 \mathrm{D}$-printed through selective laser sintering, which results in an enhanced $Z T$. Moving forward, to realize widespread commercial interest, several key factors need to be taken into account in general: low-cost and scalable processing methods, earth-abundant and non-toxic materials to ensure sustainability, easy device integration (i.e. ensuring adequate performance on the device level). Therefore, any breakthrough in any of the aforementioned aspects will 
Table 1 Summary of properties of TE materials prepared via various bottom-up methods

\begin{tabular}{|c|c|c|c|c|c|c|c|c|c|}
\hline Process type & TE Materials & $\begin{array}{l}\text { Mechanism } \\
\text { on property } \\
\text { improvement }\end{array}$ & $\sigma\left(\mathrm{S} \mathrm{cm}^{-1}\right)$ & $S\left(\mu \mathrm{V} \mathrm{K}^{-1}\right)$ & $\kappa\left(\mathrm{Wm}^{-1} \mathrm{~K}^{-1}\right)$ & $\begin{array}{l}P F(\mu \mathrm{W} \\
\left.\mathrm{m}^{-1} \mathrm{~K}^{-2}\right)\end{array}$ & $\mathrm{ZT}_{\max }$ & Temp. (K) & Refs. \\
\hline \multicolumn{10}{|c|}{ OD nanoparticles \& nanoinclusion } \\
\hline \multirow[t]{3}{*}{$\begin{array}{l}\text { Colloidal syn- } \\
\text { thesis }\end{array}$} & $\begin{array}{l}\text { PbSe quantum dot } \\
\text { films }\end{array}$ & $\begin{array}{l}\text { Quantum } \\
\text { confinement, } \\
\text { phonon scat- } \\
\text { tering }\end{array}$ & $\sim 240$ & $\sim 425$ & $\sim 1.15$ & - & 1.37 & 400 & {$[83]$} \\
\hline & $\begin{array}{l}\mathrm{PbTe} / \mathrm{PbS} \\
\text { nanocrystal films }\end{array}$ & $\begin{array}{l}\text { Quantum } \\
\text { confinement, } \\
\text { phonon scat- } \\
\text { tering }\end{array}$ & $\sim 21$ & $\sim 490$ & $\sim 0.675$ & $\sim 450$ & 0.3 & 405 & [84] \\
\hline & $\begin{array}{l}\text { PbTe@PbS core- } \\
\text { shell nanoparti- } \\
\text { cle pellets }\end{array}$ & $\begin{array}{l}\text { Phonon scat- } \\
\text { tering }\end{array}$ & 125 & -185 & 0.53 & - & 1.03 & 710 & [86] \\
\hline \multirow[t]{2}{*}{$\begin{array}{l}\text { Hydrothermal } \\
\text { synthesis }\end{array}$} & $\begin{array}{l}\text { SnTe nanocrystal } \\
\text { pellets }\end{array}$ & $\begin{array}{l}\text { Phonon scat- } \\
\text { tering energy } \\
\text { filtering }\end{array}$ & 600 & 90 & 0.60 & 350 & 0.49 & 803 & [91] \\
\hline & $\begin{array}{l}\mathrm{Cu}_{2} \mathrm{~S} \text { microcrystal } \\
\text { pellets }\end{array}$ & $\begin{array}{l}\text { Phonon scat- } \\
\text { tering }\end{array}$ & 100 & 120 & 0.2 & - & 0.38 & 573 & [92] \\
\hline \multirow[t]{2}{*}{$\begin{array}{l}\text { Electrodeposi- } \\
\text { tion process }\end{array}$} & $\begin{array}{l}\mathrm{Bi}_{2} \mathrm{Te}_{3} \text { nanocrys- } \\
\text { tals on PEDOT } \\
\text { films }\end{array}$ & $\begin{array}{l}\text { Phonon scat- } \\
\text { tering }\end{array}$ & 691 & -146 & - & 1473 & - & 298 & [96] \\
\hline & $\begin{array}{l}\text { Gold nano- } \\
\text { particles-BiTe } \\
\text { composite pellets }\end{array}$ & $\begin{array}{l}\text { Phonon scat- } \\
\text { tering }\end{array}$ & 71 & -380 & 0.5 & - & 0.62 & 298 & [98] \\
\hline \multirow[t]{3}{*}{ Nanoinclusion } & $\begin{array}{l}\text { Pt nanoparticles in } \\
\mathrm{Bi}_{2} \mathrm{Te}_{2.7} \mathrm{Se}_{0.3} \text { thin } \\
\text { films }\end{array}$ & $\begin{array}{l}\text { Energy filtering, } \\
\text { phonon scat- } \\
\text { tering }\end{array}$ & 720 & 220 & - & 3510 & - & 298 & [107] \\
\hline & $\begin{array}{c}\text { Ag nanoparticles } \\
\text { in } \mathrm{Bi}_{2} \mathrm{Te}_{3} \text { nano- } \\
\text { powder pellets }\end{array}$ & $\begin{array}{l}\text { Energy filtering, } \\
\text { phonon scat- } \\
\text { tering }\end{array}$ & 525 & -140 & 0.58 & - & 0.77 & 475 & [108] \\
\hline & $\begin{array}{l}\text { Ag nanocrystals in } \\
\text { PbS nanocrystal } \\
\text { pellets }\end{array}$ & $\begin{array}{l}\text { Energy filtering, } \\
\text { phonon scat- } \\
\text { tering }\end{array}$ & 660 & -200 & 0.8 & $>1000$ & 1.7 & 850 & [109] \\
\hline \multicolumn{10}{|c|}{ 1D Nanowires/nanofibers/nanotubes } \\
\hline \multirow[t]{6}{*}{$\begin{array}{l}\text { Solution-phase } \\
\text { synthesis }\end{array}$} & $\mathrm{Bi}_{2} \mathrm{Te}_{3} \mathrm{NW}$ pellets & $\begin{array}{l}\text { Phonon scat- } \\
\text { tering }\end{array}$ & 430 & -240 & 1.0 & 2520 & 0.96 & 380 & [116] \\
\hline & PbTe NW pellets & $\begin{array}{l}\text { Phonon scat- } \\
\text { tering }\end{array}$ & 95 & 295 & 0.9 & 850 & 0.33 & 350 & [117] \\
\hline & $\begin{array}{l}\mathrm{Bi}_{2} \mathrm{Te}_{2.7} \mathrm{Se}_{0.3} \mathrm{NW} \\
\text { pellets }\end{array}$ & $\begin{array}{l}\text { Phonon scat- } \\
\text { tering }\end{array}$ & 465 & -160 & 0.46 & 1023 & 0.75 & 320 & [122] \\
\hline & $\begin{array}{l}\mathrm{Te}-\mathrm{Bi}_{2} \mathrm{Te}_{3} \mathrm{NW} \\
\text { pellets }\end{array}$ & $\begin{array}{l}\text { Energy filtering, } \\
\text { phonon scat- } \\
\text { tering }\end{array}$ & 5.244 & 588 & 0.309 & $\sim 300$ & 0.236 & 400 & [120] \\
\hline & $\begin{array}{l}\mathrm{PbTe}-\mathrm{Bi}_{2} \mathrm{Te}_{3} \mathrm{NW} \\
\text { pellets }\end{array}$ & $\begin{array}{l}\text { Energy filtering, } \\
\text { phonon scat- } \\
\text { tering }\end{array}$ & 100 & -310 & 0.51 & $\sim 950$ & 1.2 & 620 & [124] \\
\hline & $\begin{array}{l}\mathrm{Ag}_{2} \mathrm{Te}-\mathrm{Bi}_{2} \mathrm{Te}_{3} \mathrm{NW} \\
\text { pellets }\end{array}$ & $\begin{array}{l}\text { Energy filtering, } \\
\text { phonon scat- } \\
\text { tering }\end{array}$ & 28 & 275 & 0.2 & 210 & 0.41 & 400 & [123] \\
\hline $\begin{array}{l}\text { Template- } \\
\text { assisted elec- } \\
\text { trodeposition } \\
\text { method }\end{array}$ & $\begin{array}{c}\mathrm{Bi}_{0.5} \mathrm{Sb}_{1.5} \mathrm{Te}_{3} \mathrm{NW} \\
\text { membranes }\end{array}$ & $\begin{array}{l}\text { Phonon scat- } \\
\text { tering }\end{array}$ & 78 & 138 & - & 153 & - & 298 & [143] \\
\hline
\end{tabular}


Table 1 (continued)

\begin{tabular}{|c|c|c|c|c|c|c|c|c|c|}
\hline Process type & TE Materials & $\begin{array}{l}\text { Mechanism } \\
\text { on property } \\
\text { improvement }\end{array}$ & $\sigma\left(\mathrm{S} \mathrm{cm}^{-1}\right)$ & $S\left(\mu \mathrm{V} \mathrm{K}^{-1}\right)$ & $\kappa\left(\mathrm{Wm}^{-1} \mathrm{~K}^{-1}\right)$ & $\begin{array}{l}P F(\mu \mathrm{W} \\
\left.\mathrm{m}^{-1} \mathrm{~K}^{-2}\right)\end{array}$ & $\mathrm{ZT}_{\max }$ & Temp. (K) & Refs. \\
\hline & $\begin{array}{l}\mathrm{Bi}_{38} \mathrm{Te}_{55} \mathrm{Se}_{7} \mathrm{NW} \\
\text { thin films }\end{array}$ & $\begin{array}{l}\text { Phonon scat- } \\
\text { tering }\end{array}$ & 2200 & -115 & - & 2820 & - & 298 & [149] \\
\hline & $\begin{array}{c}\mathrm{Bi}_{15} \mathrm{Sb}_{29} \mathrm{Te}_{56} \mathrm{NW} \\
\text { thin films }\end{array}$ & $\begin{array}{l}\text { Phonon scat- } \\
\text { tering }\end{array}$ & 720 & 156 & - & 1750 & - & 298 & [149] \\
\hline Electrospinning & $\begin{array}{l}\mathrm{PbTe} \text { nanotube } \\
\text { mats }\end{array}$ & $\begin{array}{l}\text { Quantum } \\
\text { confinement, } \\
\text { phonon scat- } \\
\text { tering }\end{array}$ & 0.148 & 196 & - & 0.567 & - & 298 & [163] \\
\hline \multicolumn{10}{|c|}{ 2D Nanoflake/nanosheet/nanoplate } \\
\hline $\begin{array}{l}\text { Chemical vapor } \\
\text { deposition }\end{array}$ & $\begin{array}{l}\text { Ag-doped } \mathrm{SnSe} \\
\text { nanoflake thin } \\
\text { films }\end{array}$ & $\begin{array}{l}\text { Phonon scat- } \\
\text { tering }\end{array}$ & 5 & 370 & - & 66 & - & 300 & [177] \\
\hline $\begin{array}{l}\text { Molecular beam } \\
\text { epitaxy }\end{array}$ & $\begin{array}{l}\mathrm{Sb}_{2.31} \mathrm{Te}_{3} \text { thin } \\
\text { films }\end{array}$ & $\begin{array}{l}\text { Phonon scat- } \\
\text { tering }\end{array}$ & 1810 & 118 & - & 2520 & - & 298 & [224] \\
\hline $\begin{array}{l}\text { Hydrothermal } \\
\text { method }\end{array}$ & $\begin{array}{l}\text { Ge-doped } \mathrm{SnSe} \\
\text { nanoplate pellets }\end{array}$ & $\begin{array}{l}\text { Phonon scat- } \\
\text { tering }\end{array}$ & 70 & 275 & 0.18 & 510 & 2.1 & 873 & [192] \\
\hline \multirow[t]{4}{*}{$\begin{array}{l}\text { Solvothermal } \\
\text { method }\end{array}$} & $\begin{array}{l}\mathrm{Bi}_{2} \mathrm{Te}_{3} \text { nanoplate } \\
\text { films }\end{array}$ & $\begin{array}{l}\text { Phonon scat- } \\
\text { tering }\end{array}$ & 122 & -103 & - & 128 & - & 298 & [196] \\
\hline & $\begin{array}{l}\mathrm{Bi}_{2} \mathrm{Te}_{3} \text { nanoplate } \\
\text { pellets }\end{array}$ & $\begin{array}{l}\text { Phonon scat- } \\
\text { tering }\end{array}$ & 600 & -137 & 0.1 & 1057 & 0.97 & 420 & [197] \\
\hline & $\begin{array}{l}\mathrm{Bi}_{2} \mathrm{Te}_{3} \text { nanosheet } \\
\text { pellets }\end{array}$ & $\begin{array}{l}\text { Phonon scat- } \\
\text { tering }\end{array}$ & 735 & -180 & 1.2 & 2400 & 0.69 & 333 & [225] \\
\hline & $\begin{array}{l}\mathrm{Bi}_{2} \mathrm{Te}_{2.7} \mathrm{Se}_{0.3} \text { nano- } \\
\text { plate pellets }\end{array}$ & $\begin{array}{l}\text { Phonon scat- } \\
\text { tering }\end{array}$ & 480 & -198 & 0.72 & 1875 & 1.23 & 480 & [198] \\
\hline \multirow[t]{3}{*}{$\begin{array}{l}\text { Solution-phase } \\
\text { synthesis }\end{array}$} & $\begin{array}{l}\text { SnSe nanoplate } \\
\text { pellets }\end{array}$ & $\begin{array}{l}\text { Phonon scat- } \\
\text { tering }\end{array}$ & 31 & 320 & 0.55 & 375 & 1.05 & 805 & [226] \\
\hline & $\begin{array}{l}\mathrm{Sn}_{0.94} \mathrm{Bi}_{0.06} \mathrm{Se} \\
\text { nanosheet pellets }\end{array}$ & $\begin{array}{l}\text { Phonon scat- } \\
\text { tering }\end{array}$ & 12.5 & -285 & 0.3 & 100 & 0.21 & 719 & [201] \\
\hline & $\begin{array}{l}\text { SnSe nanoplate } \\
\text { pellet }\end{array}$ & $\begin{array}{l}\text { Phonon scat- } \\
\text { tering }\end{array}$ & 35 & 340 & - & 400 & - & 550 & [204] \\
\hline \multicolumn{10}{|c|}{$3 \mathrm{D}$ porous nanostructure } \\
\hline \multirow[t]{3}{*}{$\begin{array}{l}\text { Random porous } \\
\text { structure }\end{array}$} & $\begin{array}{l}\mathrm{PbS} \text { nanocrystal } \\
\text { monolith }\end{array}$ & $\begin{array}{l}\text { Phonon scat- } \\
\text { tering }\end{array}$ & $\sim 400$ & $\sim 187$ & 0.56 & 1375 & 1.06 & 838 & [207] \\
\hline & $\begin{array}{l}\mathrm{Bi}_{0.5} \mathrm{Sb}_{1.5} \mathrm{Te}_{3} \text { pow- } \\
\text { der monolith }\end{array}$ & $\begin{array}{l}\text { Phonon scat- } \\
\text { tering }\end{array}$ & 403 & 173 & 0.27 & 1100 & 1.29 & 327 & [208] \\
\hline & $\begin{array}{l}\text { Nanoporous } \mathrm{SnSe} \\
\text { pellets }\end{array}$ & $\begin{array}{l}\text { Phonon scat- } \\
\text { tering }\end{array}$ & $\sim 40$ & $\sim 325$ & 0.24 & 506 & 1.7 & 823 & [217] \\
\hline \multirow{2}{*}{$\begin{array}{l}\text { Template- } \\
\text { assisted } \\
\text { uniform porous } \\
\text { structure }\end{array}$} & $\mathrm{Bi}_{1.5} \mathrm{Sb}_{0.5} \mathrm{Te}_{3}$ film & $\begin{array}{l}\text { Phonon scat- } \\
\text { tering }\end{array}$ & 644.5 & 144 & 0.89 & 1250 & 0.56 & 400 & [209] \\
\hline & $\mathrm{ZnO}$ film & $\begin{array}{l}\text { Phonon scat- } \\
\text { tering }\end{array}$ & $\sim 45$ & $\sim 253$ & $\sim 3.6$ & 290 & 0.072 & 693 & [210] \\
\hline \multicolumn{10}{|l|}{ Nanoscale doping } \\
\hline $\begin{array}{l}\text { Hydrothermal } \\
\text { method }\end{array}$ & $\begin{array}{l}\mathrm{Pb}, \text { Cd-doped } \\
\text { poly-crystalline } \\
\mathrm{SnSe}\end{array}$ & $\begin{array}{l}\text { Phonon scat- } \\
\text { tering, carrier } \\
\text { concentration }\end{array}$ & $\sim 85$ & $\sim 285$ & 0.23 & 750 & 1.9 & 873 & [215] \\
\hline $\begin{array}{l}\text { Hydrothermal } \\
\text { method }\end{array}$ & $\begin{array}{l}\mathrm{Pb}, \mathrm{S} \text {-doped poly- } \\
\text { crystalline } \mathrm{SnSe}\end{array}$ & $\begin{array}{l}\text { Phonon scat- } \\
\text { tering, carrier } \\
\text { concentration }\end{array}$ & $\sim 38$ & $\sim 325$ & 0.13 & 418 & 1.85 & 873 & [218] \\
\hline
\end{tabular}


Table 1 (continued)

\begin{tabular}{|c|c|c|c|c|c|c|c|c|c|}
\hline Process type & TE Materials & $\begin{array}{l}\text { Mechanism } \\
\text { on property } \\
\text { improvement }\end{array}$ & $\sigma\left(\mathrm{S} \mathrm{cm}^{-1}\right)$ & $S\left(\mu \mathrm{V} \mathrm{K}^{-1}\right)$ & $\kappa\left(\mathrm{Wm}^{-1} \mathrm{~K}^{-1}\right)$ & $\begin{array}{l}P F(\mu \mathrm{W} \\
\left.\mathrm{m}^{-1} \mathrm{~K}^{-2}\right)\end{array}$ & $\mathrm{ZT}_{\text {max }}$ & Temp. (K) & Refs. \\
\hline $\begin{array}{l}\text { Hydrothermal } \\
\text { method }\end{array}$ & $\begin{array}{l}\text { Se quantum dot/ } \\
\mathrm{Sn}_{0.99} \mathrm{~Pb}_{0.01} \mathrm{Se} \\
\text { nanocomposite }\end{array}$ & $\begin{array}{l}\text { Phonon scat- } \\
\text { tering, energy } \\
\text { filtering }\end{array}$ & $\sim 31$ & $\sim 420$ & 0.245 & 560 & 2.0 & 873 & [214] \\
\hline $\begin{array}{l}\text { Solvothermal } \\
\text { method }\end{array}$ & $\begin{array}{l}\text { Cd-doped poly- } \\
\text { crystalline SnSe }\end{array}$ & $\begin{array}{l}\text { Phonon scat- } \\
\text { tering, carrier } \\
\text { concentration }\end{array}$ & 79 & 295 & 0.33 & 690 & 1.7 & 823 & [227] \\
\hline $\begin{array}{l}\text { Solvothermal } \\
\text { method }\end{array}$ & $\begin{array}{l}\mathrm{Cu} \text {-doped poly- } \\
\text { crystalline } \mathrm{SnSe}\end{array}$ & $\begin{array}{l}\text { Phonon scat- } \\
\text { tering, carrier } \\
\text { mobility }\end{array}$ & 56 & 316 & 0.32 & 557 & 1.41 & 823 & [218] \\
\hline
\end{tabular}

undoubtedly be an important step forward towards making TE devices more competitive.

Acknowledgement The authors acknowledge support from A*STAR's Science and Engineering Research Council, PHAROS program on Hybrid Thermoelectrics for Ambient Applications (Grant Nos.: 1527200019, 1527200020 and 1527200021) and Agritech program on Sustainable Hybrid Lighting System for Controlled Environment Agriculture: A19D9a0096.

Open Access This article is licensed under a Creative Commons Attribution 4.0 International License, which permits use, sharing, adaptation, distribution and reproduction in any medium or format, as long as you give appropriate credit to the original author(s) and the source, provide a link to the Creative Commons licence, and indicate if changes were made. The images or other third party material in this article are included in the article's Creative Commons licence, unless indicated otherwise in a credit line to the material. If material is not included in the article's Creative Commons licence and your intended use is not permitted by statutory regulation or exceeds the permitted use, you will need to obtain permission directly from the copyright holder. To view a copy of this licence, visit http://creativecommons.org/licenses/by/4.0/.

\section{References}

1. J. Zhai, T. Wang, H. Wang, W. Su, X. Wang et al., Strategies for optimizing the thermoelectricity of PbTe alloys. Chin. Phys. B 27(4), 047306 (2018). https://doi.org/10.1088/16741056/27/4/047306/meta

2. H. Mamur, M.R.A. Bhuiyan, F. Korkmaz, M. Nil, A review on bismuth telluride $\left(\mathrm{Bi}_{2} \mathrm{Te}_{3}\right)$ nanostructure for thermoelectric applications. Renew. Sust. Energ. Rev. 82, 4159-4169 (2018). https://doi.org/10.1016/j.rser.2017.10.112

3. Y. Zheng, H. Xie, Q. Zhang, A. Suwardi, X. Cheng et al., Unraveling the critical role of melt-spinning atmosphere in enhancing the thermoelectric performance of p-type $\mathrm{Bi}_{0.52} \mathrm{Sb}_{1.48} \mathrm{Te}_{3}$ alloys. ACS Appl. Mater. Interfaces 12(32), 36186-36195 (2020). https://doi.org/10.1021/acsami.0c096 56
4. Y. Luo, S. Cai, X. Hua, H. Chen, Q. Liang et al., High thermoelectric performance in polycrystalline $\mathrm{SnSe}$ via dualdoping with $\mathrm{Ag} / \mathrm{Na}$ and nanostructuring with $\mathrm{Ag}_{8} \mathrm{SnSe}_{6}$. Adv. Energy Mater. 9(2), 1803072 (2019). https://doi.org/10.1002/ aenm.201803072

5. Z.Z. Luo, X. Zhang, X. Hua, G. Tan, T.P. Bailey et al., High thermoelectric performance in supersaturated solid solutions and nanostructured n-type PbTe-GeTe. Adv. Funct. Mater. 28(31), 1801617 (2018). https://doi.org/10.1002/adfm.20180 1617

6. Y. Luo, Y. Zheng, Z. Luo, S. Hao, C. Du et al., N-type $\mathrm{SnSe}_{2}$ oriented-nanoplate-based pellets for high thermoelectric performance. Adv. Energy Mater. 8(8), 1702167 (2018). https:// doi.org/10.1002/aenm.201702167

7. G. Tan, L.-D. Zhao, M.G. Kanatzidis, Rationally designing high-performance bulk thermoelectric materials. Chem. Rev. 116(19), 12123-12149 (2016). https://doi.org/10.1021/acs. chemrev.6b00255

8. J. Mao, Z. Liu, J. Zhou, H. Zhu, Q. Zhang et al., Advances in thermoelectrics. Adv. Phys. 67(2), 69-147 (2018). https:// doi.org/10.1080/00018732.2018.1551715

9. D.V.M. Repaka, A. Suwardi, P. Kumar, New paradigm for efficient thermoelectrics. Energy Saving Coating Mater. (2020). https://doi.org/10.1016/B978-0-12-822103-7. 00008-X

10. X.-L. Shi, J. Zou, Z.-G. Chen, Advanced thermoelectric design: From materials and structures to devices. Chem. Rev. 120(15), 7399-7515 (2020). https://doi.org/10.1021/ acs.chemrev.0c00026

11. E.S. Toberer, A. Zevalkink, G.J. Snyder, Phonon engineering through crystal chemistry. J. Mater. Chem. 21(40), 1584315852 (2011). https://doi.org/10.1039/C1JM11754H

12. J.P. Heremans, V. Jovovic, E.S. Toberer, A. Saramat, K. Kurosaki et al., Enhancement of thermoelectric efficiency in $\mathrm{PbTe}$ by distortion of the electronic density of states. Science 321(5888), 554-557 (2008). https://doi.org/10.1126/science. 1159725

13. Y. Pei, X. Shi, A. LaLonde, H. Wang, L. Chen et al., Convergence of electronic bands for high performance bulk thermoelectrics. Nature 473(7345), 66-69 (2011). https://doi.org/10. 1038/nature09996 
14. L.-D. Zhao, S.-H. Lo, Y. Zhang, H. Sun, G. Tan et al., Ultralow thermal conductivity and high thermoelectric figure of merit in SnSe crystals. Nature 508(7496), 373-377 (2014). https://doi.org/10.1038/nature13184

15. J. Recatala-Gomez, A. Suwardi, I. Nandhakumar, A. Abutaha, K. Hippalgaonkar, Toward accelerated thermoelectric materials and process discovery. ACS Appl. Energy Mater. 3(3), 2240-2257 (2020). https://doi.org/10.1021/acsaem.9b02222

16. A. Suwardi, J. Cao, L. Hu, F. Wei, J. Wu et al., Tailoring the phase transition temperature to achieve high-performance cubic GeTe-based thermoelectrics. J. Mater. Chem. A 8(36), 18880-18890 (2020). https://doi.org/10.1039/D0TA06013E

17. Y. Pei, A.D. LaLonde, H. Wang, G.J. Snyder, Low effective mass leading to high thermoelectric performance. Energy Environ. Sci. 5(7), 7963-7969 (2012). https://doi.org/10. 1039/C2EE21536E

18. A. Suwardi, D. Bash, H.K. Ng, J.R. Gomez, D.M. Repaka et al., Inertial effective mass as an effective descriptor for thermoelectrics via data-driven evaluation. J. Mater. Chem. A 7(41), 23762-23769 (2019). https://doi.org/10.1039/C9TA0 5967A

19. M. Dresselhaus, Y. Lin, G. Dresselhaus, X. Sun, Z. Zhang et al., Advances in 1D and 2D thermoelectric materials. In Eighteenth International Conference on Thermoelectrics Proceedings, ICT'99 (Cat No 99TH8407). 92-99 (1999). https:// doi.org/https://doi.org/10.1109/ICT.1999.843342

20. Y. Wu, Z. Chen, P. Nan, F. Xiong, S. Lin et al., Lattice strain advances thermoelectrics. Joule 3(5), 1276-1288 (2019). https://doi.org/10.1016/j.joule.2019.02.008

21. T.G. Novak, K. Kim, S. Jeon, 2D and 3D nanostructuring strategies for thermoelectric materials. Nanoscale 11(42), 19684-19699 (2019). https://doi.org/10.1039/C9NR07406F

22. S. Ortega, M. Ibáñez, Y. Liu, Y. Zhang, M.V. Kovalenko et al., Bottom-up engineering of thermoelectric nanomaterials and devices from solution-processed nanoparticle building blocks. Chem. Soc. Rev. 46(12), 3510-3528 (2017). https://doi.org/10.1039/C6CS00567E

23. B. Xu, T. Feng, Z. Li, W. Zheng, Y. Wu, Large-scale, solution-synthesized nanostructured composites for thermoelectric applications. Adv. Mater. 30(48), 1801904 (2018). https:// doi.org/10.1002/adma.201801904

24. H. Alam, S. Ramakrishna, A review on the enhancement of figure of merit from bulk to nano-thermoelectric materials. Nano Energy 2(2), 190-212 (2013). https://doi.org/10.1016/j. nanoen.2012.10.005

25. H.J. Goldsmid, Introduction to Thermoelectricity (Springer, Berlin, 2010).

26. J. Mao, W. Liu, Z. Ren, Carrier distribution in multi-band materials and its effect on thermoelectric properties. J. Materiomics 2(2), 203-211 (2016). https://doi.org/10.1016/j.jmat. 2016.03.001

27. Z.-G. Chen, G. Han, L. Yang, L. Cheng, J. Zou, Nanostructured thermoelectric materials: current research and future challenge. Prog. Nat. Sci. Mater. Int. 22(6), 535-549 (2012). https://doi.org/10.1016/j.pnsc.2012.11.011
28. S. Lin, W. Li, Z. Bu, B. Gao, J. Li et al., Thermoelectric properties of $\mathrm{Ag}_{9} \mathrm{GaS}_{6}$ with ultralow lattice thermal conductivity. Mater. Today Phys. 6, 60-67 (2018). https://doi.org/ 10.1016/j.mtphys.2018.09.001

29. W. Li, S. Lin, M. Weiss, Z. Chen, J. Li et al., Crystal structure induced ultralow lattice thermal conductivity in thermoelectric $\mathrm{Ag}_{9} \mathrm{AlSe}_{6}$. Adv. Energy Mater. 8(18), 1800030 (2018). https://doi.org/10.1002/aenm.201800030

30. G.J. Snyder, E.S. Toberer, Complex Thermoelectric Materials (World Scientific, Singapore, 2011), pp. 101-110

31. A. Suwardi, L. Hu, X. Wang, X.Y. Tan, D.V.M. Repaka et al., Origin of high thermoelectric performance in earth-abundant phosphide-tetrahedrite. ACS Appl. Mater. Interfaces 12(8), 9150-9157 (2020). https://doi.org/10.1021/acsami.9b17269

32. G. Chen, Nanoscale Energy Transport and Conversion: A Parallel Treatment of Electrons, Molecules, Phonons, and Photons (Oxford University Press, Oxford, 2005).

33. M. Lundstrom, J. Guo, Nanoscale Transistors: Device Physics, Modeling and Simulation (Springer, Berlin, 2006).

34. T.-H. Liu, J. Zhou, M. Li, Z. Ding, Q. Song et al., Electron mean-free-path filtering in dirac material for improved thermoelectric performance. PNAS 115(5), 879-884 (2018). https://doi.org/10.1073/pnas.1715477115

35. J.-H. Lee, J. Grossman, J. Reed, G. Galli, Lattice thermal conductivity of nanoporous Si: molecular dynamics study. Appl. Phys. Lett. 91(22), 223110 (2007). https://doi.org/10. 1063/1.2817739

36. J.D. Chung, M. Kaviany, Effects of phonon pore scattering and pore randomness on effective conductivity of porous silicon. Int. J. Heat. Mass Transf. 43(4), 521-538 (2000). https:// doi.org/10.1016/S0017-9310(99)00165-9

37. K. Biswas, J. He, I.D. Blum, C.-I. Wu, T.P. Hogan et al., High-performance bulk thermoelectrics with all-scale hierarchical architectures. Nature 489(7416), 414-418 (2012). https://doi.org/10.1038/nature11439

38. D.G. Cahill, S.K. Watson, R.O. Pohl, Lower limit to the thermal conductivity of disordered crystals. Phys. Rev. B 46(10), 6131 (1992). https://doi.org/10.1103/PhysRevB.46.6131

39. H. Wu, J. Carrete, Z. Zhang, Y. Qu, X. Shen et al., Strong enhancement of phonon scattering through nanoscale grains in lead sulfide thermoelectrics. NPG Asia Mater. 6(6), e108e108 (2014). https://doi.org/10.1038/am.2014.39

40. S.Y. Ren, J.D. Dow, Thermal conductivity of superlattices. Phys. Rev. B 25(6), 3750 (1982). https://doi.org/10.1103/ PhysRevB.25.3750

41. J. Mao, Z. Liu, Z. Ren, Size effect in thermoelectric materials. NPJ Quantum Mater. 1(1), 1-9 (2016). https://doi.org/10. 1038/npjquantmats.2016.28

42. L. Hicks, M.S. Dresselhaus, Effect of quantum-well structures on the thermoelectric figure of merit. Phys. Rev. B 47(19), 12727 (1993). https://doi.org/10.1103/PhysRevB.47.12727

43. L. Hicks, M.S. Dresselhaus, Thermoelectric figure of merit of a one-dimensional conductor. Phys. Rev. B 47(24), 16631 (1993). https://doi.org/10.1103/PhysRevB.47.16631 
44. P. Pichanusakorn, P. Bandaru, Nanostructured thermoelectrics. Mater. Sci. Eng. R Rep. 67(2-4), 19-63 (2010). https:// doi.org/10.1016/j.mser.2009.10.001

45. C.J. Vineis, A. Shakouri, A. Majumdar, M.G. Kanatzidis, Nanostructured thermoelectrics: Big efficiency gains from small features. Adv. Mater. 22(36), 3970-3980 (2010). https://doi.org/10.1002/adma.201000839

46. J.-F. Li, W.-S. Liu, L.-D. Zhao, M. Zhou, High-performance nanostructured thermoelectric materials. NPG Asia Mater. 2(4), 152-158 (2010). https://doi.org/10.1038/asiamat.2010. 138

47. M.S. Dresselhaus, G. Chen, M.Y. Tang, R. Yang, H. Lee et al., New directions for low-dimensional thermoelectric materials. Adv. Mater. 19(8), 1043-1053 (2007). https://doi. org/10.1002/adma.200600527

48. L. Hicks, T. Harman, X. Sun, M. Dresselhaus, Experimental study of the effect of quantum-well structures on the thermoelectric figure of merit. Phys. Rev. B 53(16), R10493 (1996). https://doi.org/10.1103/PhysRevB.53.R10493

49. E. Rogacheva, T. Tavrina, O. Nashchekina, S. Grigorov, K. Nasedkin et al., Quantum size effects in pbse quantum wells. Appl. Phys. Lett. 80(15), 2690-2692 (2002). https://doi.org/ 10.1063/1.1469677

50. H. Ohta, S. Kim, Y. Mune, T. Mizoguchi, K. Nomura et al., Giant thermoelectric seebeck coefficient of a two-dimensional electron gas in $\mathrm{SrTiO}_{3}$. Nat. Mater. 6(2), 129-134 (2007). https://doi.org/10.1038/nmat1821

51. A.I. Boukai, Y. Bunimovich, J. Tahir-Kheli, J.-K. Yu, W.A. Goddard Iii et al., Silicon nanowires as efficient thermoelectric materials. Nature 451(7175), 168-171 (2008). https://doi. org/10.1038/nature06458

52. P.M. Wu, J. Gooth, X. Zianni, S.F. Svensson, J.G.R. Gluschke et al., Large thermoelectric power factor enhancement observed in inas nanowires. Nano Lett. 13(9), 4080-4086 (2013). https://doi.org/10.1021/nl401501j

53. T. Harman, D. Spears, M. Manfra, High thermoelectric figures of merit in pbte quantum wells. J. Electron. Mater. 25(7), 1121-1127 (1996). https://doi.org/10.1007/BF02659913

54. T. Harman, P. Taylor, D. Spears, M. Walsh, Thermoelectric quantum-dot superlattices with high ZT. J. Electron Mater. 29(1), L1-L2 (2000). https://doi.org/10.1007/ s11664-000-0117-1

55. L. Wu, X. Li, S. Wang, T. Zhang, J. Yang et al., Resonant level-induced high thermoelectric response in indium-doped GeTe. NPG Asia Mater. 9(1), e343 (2017). https://doi.org/10. 1038/am.2016.203

56. A. Suwardi, J. Cao, Y. Zhao, J. Wu, S.W. Chien et al., Achieving high thermoelectric quality factor towards high figure of merit in GeTe. Mater. Today Phys. (2020). https://doi.org/10. 1016/j.mtphys.2020.100239

57. A.F. Ioffe, L. Stil'Bans, E. Iordanishvili, T. Stavitskaya, A. Gelbtuch et al., Semiconductor thermoelements and thermoelectric cooling. Phys. Today 12(5), 42 (1959). https://doi. org/10.1063/1.3060810
58. D. Rowe, G. Min, Multiple potential barriers as a possible mechanism to increase the Seebeck coefficient and electrical power factor. AIP Conf. Proc. 316(1), 339-342 (1994). https://doi.org/10.1063/1.46827

59. C. Li, S. Ma, P. Wei, W. Zhu, X. Nie et al., Magnetisminduced huge enhancement of the room-temperature thermoelectric and cooling performance of p-type BiSbTe alloys. Energy Environ. Sci. 13(2), 535-544 (2020). https://doi.org/ 10.1039/C9EE03446C

60. Z. Liang, M.J. Boland, K. Butrouna, D.R. Strachan, K.R. Graham, Increased power factors of organic-inorganic nanocomposite thermoelectric materials and the role of energy filtering. J. Mater. Chem. A 5(30), 15891-15900 (2017). https:// doi.org/10.1039/C7TA02307C

61. N. Mingo, D. Hauser, N. Kobayashi, M. Plissonnier, A. Shakouri, "Nanoparticle-in-alloy" approach to efficient thermoelectrics: Silicides in SiGe. Nano Lett. 9(2), 711-715 (2009). https://doi.org/10.1021/n18031982

62. W. Kim, J. Zide, A. Gossard, D. Klenov, S. Stemmer et al., Thermal conductivity reduction and thermoelectric figure of merit increase by embedding nanoparticles in crystalline semiconductors. Phys. Rev. Lett. 96(4), 045901 (2006). https://doi.org/10.1103/PhysRevLett.96.045901

63. M.-S. Jeng, R. Yang, D. Song, G. Chen, Modeling the thermal conductivity and phonon transport in nanoparticle composites using monte carlo simulation. J. Heat Transf. 130(4), 042410 (2008). https://doi.org/10.1115/1.2818765

64. A. Kundu, N. Mingo, D. Broido, D. Stewart, Role of light and heavy embedded nanoparticles on the thermal conductivity of SiGe alloys. Phys. Rev. B 84(12), 125426 (2011). https:// doi.org/10.1103/PhysRevB.84.125426

65. H. Zhang, A.J. Minnich, The best nanoparticle size distribution for minimum thermal conductivity. Sci. Rep. 5, 8995 (2015). https://doi.org/10.1038/srep08995

66. J. He, J.R. Sootsman, S.N. Girard, J.-C. Zheng, J. Wen et al., On the origin of increased phonon scattering in nanostructured pbte based thermoelectric materials. J. Am. Chem. Soc. 132(25), 8669-8675 (2010). https://doi.org/10.1021/ja101 0948

67. L.D. Zhao, H.J. Wu, S.Q. Hao, C.I. Wu, X.Y. Zhou et al., Allscale hierarchical thermoelectrics: MgTe in PbTe facilitates valence band convergence and suppresses bipolar thermal transport for high performance. Energy Environ. Sci. 6(11), 3346-3355 (2013). https://doi.org/10.1039/C3EE42187B

68. L.-D. Zhao, J. He, S. Hao, C.-I. Wu, T.P. Hogan et al., Raising the thermoelectric performance of p-type $\mathrm{PbS}$ with endotaxial nanostructuring and valence-band offset engineering using cds and ZnS. J. Am. Chem. Soc. 134(39), 16327-16336 (2012). https://doi.org/10.1021/ja306527n

69. L.-D. Zhao, J. He, C.-I. Wu, T.P. Hogan, X. Zhou et al., Thermoelectrics with earth abundant elements: High performance p-type $\mathrm{PbS}$ nanostructured with SrSand CaS. J. Am. Chem. Soc. 134(18), 7902-7912 (2012). https://doi.org/10.1021/ ja301772w

70. L.-D. Zhao, X. Zhang, H. Wu, G. Tan, Y. Pei et al., Enhanced thermoelectric properties in the counter-doped SnTe system 
with strained endotaxial SrTe. J. Am. Chem. Soc. 138(7), 2366-2373 (2016). https://doi.org/10.1021/jacs.5b13276

71. J. Recatala-Gomez, H.K. Ng, P. Kumar, A. Suwardi, M. Zheng et al., Thermoelectric properties of substoichiometric electron beam patterned bismuth sulfide. ACS Appl. Mater. Interfaces 12(30), 33647-33655 (2020). https://doi.org/10. 1021/acsami.0c06829

72. L.P. Tan, T. Sun, S. Fan, L.Y. Ng, A. Suwardi et al., Facile synthesis of $\mathrm{Cu}_{7} \mathrm{Te}_{4}$ nanorods and the enhanced thermoelectric properties of $\mathrm{Cu}_{7} \mathrm{Te}_{4}-\mathrm{Bi}_{0.4} \mathrm{Sb}_{1.6} \mathrm{Te}_{3}$ nanocomposites. Nano Energy 2(1), 4-11 (2013). https://doi.org/10.1016/j. nanoen.2012.07.004

73. X. Wang, A. Suwardi, Y. Zheng, H. Zhou, S.W. Chien et al., Enhanced thermoelectric performance of nanocrystalline indium tin oxide pellets by modulating the density and nanoporosity via spark plasma sintering. ACS Appl. Nano Mater. 3(10), 10156-10165 (2020). https://doi.org/10.1021/acsanm. 0c02113

74. A. Suwardi, S.H. Lim, Y. Zheng, X. Wang, S.W. Chien et al., Effective enhancement of thermoelectric and mechanical properties of germanium telluride via rhenium-doping. J. Mater. Chem. C 8(47), 16940-16948 (2020). https://doi.org/ 10.1039/D0TC04903D

75. R. Venkatasubramanian, E. Siivola, T. Colpitts, B. O'quinn, Thin-film thermoelectric devices with high room-temperature figures of merit. Nature 413(6856), 597-602 (2001). https:// doi.org/10.1038/35098012

76. H.-T. Chang, C.-C. Wang, J.-C. Hsu, M.-T. Hung, P.-W. Li et al., High quality multifold $\mathrm{Ge} / \mathrm{Si} / \mathrm{Ge}$ composite quantum dots for thermoelectric materials. Appl. Phys. Lett. 102(10), 101902 (2013). https://doi.org/10.1063/1.4794943

77. H.-T. Chang, S.-Y. Wang, S.-W. Lee, Designer ge/si composite quantum dots with enhanced thermoelectric properties. Nanoscale 6(7), 3593-3598 (2014). https://doi.org/10.1039/ C3NR06335F

78. A. Nadtochiy, V. Kuryliuk, V. Strelchuk, O. Korotchenkov, P.-W. Li et al., Enhancing the seebeck effect in Ge/Si through the combination of interfacial design features. Sci. Rep. 9(1), 1-11 (2019). https://doi.org/10.1038/s41598-019-52654-Z

79. J. Chang, E.R. Waclawik, Colloidal semiconductor nanocrystals: controlled synthesis and surface chemistry in organic media. RSC Adv. 4(45), 23505-23527 (2014). https://doi. org/10.1039/C4RA02684E

80. N.P. Dasgupta, J. Sun, C. Liu, S. Brittman, S.C. Andrews et al., $25^{\text {th }}$ anniversary article: semiconductor nanowires-synthesis, characterization, and applications. Adv. Mater. 26(14), 2137-2184 (2014). https://doi.org/10.1002/adma.201305929

81. X. Wang, A. Suwardi, S.L. Lim, F. Wei, J. Xu, Transparent flexible thin-film $\mathrm{p}-\mathrm{n}$ junction thermoelectric module. NPJ Flex. Electron. 4(1), 1-9 (2020)

82. R.Y. Wang, J.P. Feser, J.-S. Lee, D.V. Talapin, R. Segalman et al., Enhanced thermopower in PbSe nanocrystal quantum dot superlattices. Nano Lett. 8(8), 2283-2288 (2008). https:// doi.org/10.1021/n18009704
83. D. Yang, C. Lu, H. Yin, I.P. Herman, Thermoelectric performance of PbSe quantum dot films. Nanoscale 5(16), 72907296 (2013). https://doi.org/10.1039/C3NR01875J

84. D. Ding, D. Wang, M. Zhao, J. Lv, H. Jiang et al., Interface engineering in solution-processed nanocrystal thin films for improved thermoelectric performance. Adv. Mater. 29(1), 1603444 (2017). https://doi.org/10.1002/adma.201603444

85. M.I. Nugraha, H. Kim, B. Sun, S. Desai, F.P.G. de Arquer et al., Highly passivated n-type colloidal quantum dots for solution-processed thermoelectric generators with large output voltage. Adv. Energy Mater. 9(28), 1901244 (2019). https://doi.org/10.1002/aenm.201901244

86. M. Ibáñez, R. Zamani, S. Gorsse, J. Fan, S. Ortega et al., Core-shell nanoparticles as building blocks for the bottom-up production of functional nanocomposites: $\mathrm{PbTe}-\mathrm{PbS}$ thermoelectric properties. ACS Nano 7(3), 2573-2586 (2013). https://doi.org/10.1021/nn305971v

87. C. Wang, G. Zhang, S. Fan, Y. Li, Hydrothermal synthesis of PbSe, PbTe semiconductor nanocrystals. J. Phys. Chem. Solids 62(11), 1957-1960 (2001). https://doi.org/10.1016/ S0022-3697(01)00035-X

88. H. Zhang, L.-P. Wang, H. Xiong, L. Hu, B. Yang et al., Hydrothermal synthesis for high-quality CdTe nanocrystals. Adv. Mater. 15(20), 1712-1715 (2003). https://doi.org/10. 1002/adma.200305653

89. Q. Wang, D. Pan, S. Jiang, X. Ji, L. An et al., A solvothermal route to size-and shape-controlled CdSe and CdTe nanocrystals. J. Crystal Growth 286(1), 83-90 (2006). https://doi.org/ 10.1016/j.jcrysgro.2005.05.083

90. Q. Wang, D. Pan, S. Jiang, X. Ji, L. An et al., Luminescent $\mathrm{CdSe}$ and $\mathrm{CdSe} / \mathrm{CdS}$ core-shell nanocrystals synthesized via a combination of solvothermal and two-phase thermal routes. J. Lumin. 118(1), 91-98 (2006). https://doi.org/10.1016/j.jlu$\min .2005 .06 .010$

91. Z. Li, Y. Chen, J.-F. Li, H. Chen, L. Wang et al., Systhesizing snte nanocrystals leading to thermoelectric performance enhancement via an ultra-fast microwave hydrothermal method. Nano Energy 28, 78-86 (2016). https://doi.org/10. 1016/j.nanoen.2016.08.008

92. Y.-Q. Tang, Z.-H. Ge, J. Feng, Synthesis and thermoelectric properties of copper sulfides via solution phase methods and spark plasma sintering. Curr. Comput. Aided Drug Des. 7(5), 141 (2017). https://doi.org/10.3390/cryst7050141

93. A. Datta, G.S. Nolas, Synthesis and characterization of nanocrystalline $\mathrm{FeSb}_{2}$ for thermoelectric applications. Eur. J. Inorg. Chem. 2012(1), 55-58 (2012). https://doi.org/10. 1002/ejic.201100864

94. Z. Shao, W. Zhu, Z. Li, Q. Yang, G. Wang, One-step fabrication of cds nanoparticle-sensitized $\mathrm{TiO}_{2}$ nanotube arrays via electrodeposition. J. Phys. Chem. C 116(3), 2438-2442 (2012). https://doi.org/10.1021/jp2078117

95. S. Baeck, T. Jaramillo, G. Stucky, E. McFarland, Controlled electrodeposition of nanoparticulate tungsten oxide. Nano Lett. 2(8), 831-834 (2002). https://doi.org/10.1021/n1025 $587 \mathrm{p}$ 
96. J. Na, Y. Kim, T. Park, C. Park, E. Kim, Preparation of bismuth telluride films with high thermoelectric power factor. ACS Appl. Mater. Interfaces 8(47), 32392-32400 (2016). https://doi.org/10.1021/acsami.6b10188

97. D. Zhao, J. Chen, Z. Ren, J. Chen, Q. Song et al., Thermoelectric transport and magnetoresistance of electrochemical deposited $\mathrm{Bi}_{2} \mathrm{Te}_{3}$ films at micrometer thickness. Ceram. Int. 46(3), 3339-3344 (2020). https://doi.org/10.1016/j.ceramint. 2019.10.043

98. T.H. Nguyen, J. Enju, T. Ono, Enhancement of thermoelectric properties of bismuth telluride composite with gold nanoparticles inclusions using electrochemical co-deposition. J. Electrochem. Soc. 166(12), D508 (2019). https://doi.org/10. 1149/2.1011912jes

99. S. Wang, H. Li, R. Lu, G. Zheng, X. Tang, Metal nanoparticle decorated n-type $\mathrm{Bi}_{2} \mathrm{Te}_{3}$-based materials with enhanced thermoelectric performances. Nanotechnology 24(28), 285702 (2013)

100. K. Biswas, J. He, I.D. Blum, C.-I. Wu, T.P. Hogan et al., High-performance bulk thermoelectrics with all-scale hierarchical architectures. Nature 490(7421), 570-570 (2012)

101. D. Jung, K. Kurosaki, S. Seino, M. Ishimaru, K. Sato et al., Thermoelectric properties of au nanoparticle-supported $\mathrm{Sb}_{1.6} \mathrm{Bi}_{0.4} \mathrm{Te}_{3}$ synthesized by a $\gamma$-ray irradiation method. Phys. Status Solidi B 251(1), 162-167 (2014). https://doi.org/10. 1002/pssb.201349226

102. S. Fan, J. Zhao, Q. Yan, J. Ma, H.H. Hng, Influence of nanoinclusions on thermoelectric properties of n-type $\mathrm{Bi}_{2} \mathrm{Te}_{3}$ nanocomposites. J. Electron. Mater. 40(5), 1018-1023 (2011). https://doi.org/10.1007/s11664-010-1487-7

103. Z. He, C. Stiewe, D. Platzek, G. Karpinski, E. Müller et al., Effect of ceramic dispersion on thermoelectric properties of nano- $\mathrm{ZrO}_{2} / \mathrm{CoSb}_{3}$ composites. J. Appl. Phys. 101(4), 043707 (2007). https://doi.org/10.1063/1.2561628

104. X. Zhou, G. Wang, L. Zhang, H. Chi, X. Su et al., Enhanced thermoelectric properties of Ba-filled skutterudites by grain size reduction and Ag nanoparticle inclusion. J. Mater. Chem. 22(7), 2958-2964 (2012). https://doi.org/10.1039/C2JM1 $5010 \mathrm{G}$

105. J. Li, Q. Tan, J.F. Li, D.W. Liu, F. Li et al., BiSbTe-based nanocomposites with high zt: the effect of sic nanodispersion on thermoelectric properties. Adv. Funct. Mater. 23(35), 4317-4323 (2013). https://doi.org/10.1002/adfm.201300146

106. S. Gupta, D.C. Agarwal, B. Sivaiah, S. Amrithpandian, K. Asokan et al., Enhancement in thermoelectric properties due to $\mathrm{Ag}$ nanoparticles incorporated in $\mathrm{Bi}_{2} \mathrm{Te}_{3}$ matrix. Beilstein J. Nanotechnol. 10(1), 634-643 (2019). https://doi.org/10. 3762/bjnano. 10.63

107. T. Sun, M.K. Samani, N. Khosravian, K.M. Ang, Q. Yan et al., Enhanced thermoelectric properties of n-type $\mathrm{Bi}_{2} \mathrm{Te}_{2.7} \mathrm{Se}_{0.3}$ thin films through the introduction of Pt nanoinclusions by pulsed laser deposition. Nano Energy 8, 223-230 (2014). https://doi.org/10.1016/j.nanoen.2014.06.011

108. Q. Zhang, X. Ai, L. Wang, Y. Chang, W. Luo et al., Improved thermoelectric performance of silver nanoparticles-dispersed $\mathrm{Bi}_{2} \mathrm{Te}_{3}$ composites deriving from hierarchical two-phased heterostructure. Adv. Funct. Mater. 25(6), 966-976 (2015). https://doi.org/10.1002/adfm.201402663

109. M. Ibánez, Z. Luo, A. Genc, L. Piveteau, S. Ortega et al., High-performance thermoelectric nanocomposites from nanocrystal building blocks. Nat. Commun. 7(1), 1-7 (2016). https://doi.org/10.1038/ncomms 10766

110. H. Yang, E. Wong, T. Zhao, J.D. Lee, H.L. Xin et al., Charge transport modulation in $\mathrm{PbSe}$ nanocrystal solids by $\mathrm{Au}_{\mathrm{x}} \mathrm{Ag}_{1-\mathrm{x}}$ nanoparticle doping. ACS Nano 12(9), 9091-9100 (2018). https://doi.org/10.1021/acsnano.8b03112

111. A. Tarachand, B. Mukherjee, M. Saxena, Y.-K. Kuo, G.S. Okram et al., Ag-nanoinclusion-induced enhanced thermoelectric properties of $\mathrm{Ag}_{2} \mathrm{~S}$. ACS Appl. Energy Mater. 2(9), 6383-6394 (2019). https://doi.org/10.1021/acsaem.9b01016

112. K.H. Lim, K.W. Wong, Y. Liu, Y. Zhang, D. Cadavid et al., Critical role of nanoinclusions in silver selenide nanocomposites as a promising room temperature thermoelectric material. J. Mater. Chem. C 7(9), 2646-2652 (2019). https://doi.org/ 10.1039/C9TC00163H

113. R. Chen, J. Lee, W. Lee, D. Li, Thermoelectrics of nanowires. Chem. Rev. 119(15), 9260-9302 (2019). https://doi.org/10. 1021/acs.chemrev.8b00627

114. Y. Qi, Z. Wang, M. Zhang, F. Yang, X. Wang, Thermoelectric devices based on one-dimensional nanostructures. J. Mater. Chem. A 1(20), 6110-6124 (2013). https://doi.org/10.1039/ C3TA01594G

115. K. Wang, H.-W. Liang, W.-T. Yao, S.-H. Yu, Templating synthesis of uniform $\mathrm{Bi}_{2} \mathrm{Te}_{3}$ nanowires with high aspect ratio in triethylene glycol (TEG) and their thermoelectric performance. J. Mater. Chem. 21(38), 15057-15062 (2011). https:// doi.org/10.1039/C1JM12384J

116. G. Zhang, B. Kirk, L.A. Jauregui, H. Yang, X. Xu et al., Rational synthesis of ultrathin n-type $\mathrm{Bi}_{2} \mathrm{Te}_{3}$ nanowires with enhanced thermoelectric properties. Nano Lett. 12(1), 56-60 (2012). https://doi.org/10.1021/nl202935k

117. S.W. Finefrock, G. Zhang, J.-H. Bahk, H. Fang, H. Yang et al., Structure and thermoelectric properties of spark plasma sintered ultrathin pbte nanowires. Nano Lett. 14(6), 34663473 (2014). https://doi.org/10.1021/nl500997w

118. H.-W. Liang, S. Liu, Q.-S. Wu, S.-H. Yu, An efficient templating approach for synthesis of highly uniform cdte and PbTe nanowires. Inorg. Chem. 48(11), 4927-4933 (2009). https://doi.org/10.1021/ic900245w

119. K. Wang, Y. Yang, H.-W. Liang, J.-W. Liu, S.-H. Yu, First sub-kilogram-scale synthesis of high quality ultrathin tellurium nanowires. Mater. Horizons 1(3), 338-343 (2014). https://doi.org/10.1039/C4MH00004H

120. G. Zhang, H. Fang, H. Yang, L.A. Jauregui, Y.P. Chen et al., Design principle of telluride-based nanowire heterostructures for potential thermoelectric applications. Nano Lett. 12(7), 3627-3633 (2012). https://doi.org/10.1021/nl301327d

121. Z. Xiong, Y. Cai, X. Ren, B. Cao, J. Liu et al., Solutionprocessed $\mathrm{CdS} / \mathrm{Cu}_{2} \mathrm{~S}$ superlattice nanowire with enhanced thermoelectric property. ACS Appl. Mater. Interfaces 9(38), 32424-32429 (2017). https://doi.org/10.1021/acsami.7b093 46 
122. C. Zhou, C. Dun, K. Wang, X. Zhang, Z. Shi et al., General method of synthesis ultrathin ternary metal chalcogenide nanowires for potential thermoelectric applications. Nano Energy 30, 709-716 (2016). https://doi.org/10.1016/j.nanoen. 2016.10.043

123. H. Fang, H. Yang, Y. Wu, Thermoelectric properties of silver telluride-bismuth telluride nanowire heterostructure synthesized by site-selective conversion. Chem. Mater. 26(10), 3322-3327 (2014). https://doi.org/10.1021/cm501188c

124. H. Fang, T. Feng, H. Yang, X. Ruan, Y. Wu, Synthesis and thermoelectric properties of compositional-modulated lead telluride-bismuth telluride nanowire heterostructures. Nano Lett. 13(5), 2058-2063 (2013). https://doi.org/10.1021/nl400 $319 \mathrm{u}$

125. H.-J. Choi, in Vapor-liquid-solid Growth of Semiconductor Nanowires. ed. by (Springer; 2012), pp. 1-36. https://doi.org/ 10.1007/978-3-642-22480-5_1

126. K.W. Kolasinski, Catalytic growth of nanowires: vaporliquid-solid, vapor-solid-solid, solution-liquid-solid and solid-liquid-solid growth. Curr. Opin. Solid State Mater. Sci. 10(3-4), 182-191 (2006). https://doi.org/10.1016/j.cossms. 2007.03.002

127. D. Li, Y. Wu, P. Kim, L. Shi, P. Yang et al., Thermal conductivity of individual silicon nanowires. Appl. Phys. Lett. 83(14), 2934-2936 (2003). https://doi.org/10.1063/1.16169 81

128. D. Li, Y. Wu, R. Fan, P. Yang, A. Majumdar, Thermal conductivity of $\mathrm{Si} / \mathrm{SiGe}$ superlattice nanowires. Appl. Phys. Lett. 83(15), 3186-3188 (2003). https://doi.org/10.1063/1.16192 21

129. I.D. Noyan, G. Gadea, M. Salleras, M. Pacios, C. Calaza et al., SiGe nanowire arrays based thermoelectric microgenerator. Nano Energy 57, 492-499 (2019). https://doi.org/10. 1016/j.nanoen.2018.12.050

130. Y.-H. Park, J. Kim, H. Kim, I. Kim, K.-Y. Lee et al., Thermal conductivity of VLS-grown rough si nanowires with various surface roughnesses and diameters. Appl. Phys. A 104(1), 7 (2011). https://doi.org/10.1007/s00339-011-6474-1

131. H. Kim, Y.-H. Park, I. Kim, J. Kim, H.-J. Choi et al., Effect of surface roughness on thermal conductivity of VLS-grown rough $\mathrm{Si}_{1-\mathrm{x}} \mathrm{Ge}_{\mathrm{x}}$ nanowires. Appl. Phys. A 104(1), 23-28 (2011). https://doi.org/10.1007/s00339-011-6475-0

132. D. Dávila, A. Tarancón, C. Calaza, M. Salleras, M. Fernández-Regúlez et al., Improved thermal behavior of multiple linked arrays of silicon nanowires integrated into planar thermoelectric microgenerators. J. Electron. Mater. 42(7), 19181925 (2013). https://doi.org/10.1007/s11664-013-2470-x

133. D. Dávila, R. Huber, C. Hierold, Bottom-up silicon nanowirebased thermoelectric microgenerators. J. Phys. Conf. Series 660(1), 012101 (2015)

134. D.J. Hill, T.S. Teitsworth, S. Kim, J.D. Christesen, J.F. Cahoon, Encoding highly nonequilibrium boron concentrations and abrupt morphology in p-type/n-type silicon nanowire superlattices. ACS Appl. Mater. Interfaces 9(42), 37105-37111 (2017). https://doi.org/10.1021/acsami.7b081 62
135. F. Nasirpouri, in Template Electrodeposition of Nanowires Arrays. ed. by (Springer; 2017), pp. 187-259. https://doi.org/ 10.1007/978-3-319-44920-3_5

136. L. Li, S. Xu, G. Li, Enhancement of thermoelectric properties in $\mathrm{Bi}-\mathrm{Sb}-\mathrm{Te}$ alloy nanowires by pulsed electrodeposition. Energy Technol. 3(8), 825-829 (2015). https://doi.org/10. 1002/ente.201500071

137. R.D. Reeves, L.A. Crosser, G.E. Chester, J.J. Hill, Thermoelectric property enhancement via pore confinement in template grown bismuth telluride nanowire arrays. Nanotechnology 28(50), 505401 (2017)

138. M. Proenca, M. Rosmaninho, P. Resende, C. Sousa, J. Ventura et al., Tailoring bi-te based nanomaterials by electrodeposition: Morphology and crystalline structure. Mater. Des. 118, 168-174 (2017). https://doi.org/10.1016/j.matdes.2017.01. 020

139. J. Lee, S. Farhangfar, J. Lee, L. Cagnon, R. Scholz et al., Tuning the crystallinity of thermoelectric $\mathrm{Bi}_{2} \mathrm{Te}_{3}$ nanowire arrays grown by pulsed electrodeposition. Nanotechnology 19(36), 365701 (2008)

140. J. Martín, C.V. Manzano, O. Caballero-Calero, M. MartínGonzález, High-aspect-ratio and highly ordered 15-nm porous alumina templates. ACS Appl. Mater. Interfaces 5(1), 72-79 (2013). https://doi.org/10.1021/am3020718

141. J. Krieg, R. Giraud, H. Funke, J. Dufouleur, W. Escoffier et al., Magnetotransport measurements on $\mathrm{Bi}_{2} \mathrm{Te}_{3}$ nanowires electrodeposited in etched ion-track membranes. J. Phys. Chem. Solids 128, 360-366 (2019). https://doi.org/10.1016/j. jpcs.2018.02.002

142. D. Kojda, R. Mitdank, A. Mogilatenko, W. Töllner, Z. Wang et al., The effect of a distinct diameter variation on the thermoelectric properties of individual $\mathrm{Bi}_{0.39} \mathrm{Te}_{0.61}$ nanowires. Semicond. Sci. Technol. 29(12), 124006 (2014)

143. M. Muñoz Rojo, S. Grauby, J.-M. Rampnoux, O. CaballeroCalero, M. Martín-González et al., Fabrication of $\mathrm{Bi}_{2} \mathrm{Te}_{3}$ nanowire arrays and thermal conductivity measurement by $3 \omega$-scanning thermal microscopy. J. Appl. Phys. 113(5), 054308 (2013). https://doi.org/10.1063/1.4790363

144. A. Mavrokefalos, A.L. Moore, M.T. Pettes, L. Shi, W. Wang et al., Thermoelectric and structural characterizations of individual electrodeposited bismuth telluride nanowires. J. Appl. Phys. 105(10), 104318 (2009). https://doi.org/10.1063/1. 3133145

145. M.M. Rojo, B. Abad, C.V. Manzano, P. Torres, X. Cartoixà et al., Thermal conductivity of $\mathrm{Bi}_{2} \mathrm{Te}_{3}$ nanowires: how size affects phonon scattering. Nanoscale 9(20), 6741-6747 (2017). https://doi.org/10.1039/C7NR02173A

146. C. De Tomas, A. Cantarero, A. Lopeandia, F. Alvarez, From kinetic to collective behavior in thermal transport on semiconductors and semiconductor nanostructures. J. Appl. Phys. 115(16), 164314 (2014). https://doi.org/10.1063/1.4871672

147. P. Kumar, M. Pfeffer, N. Peranio, O. Eibl, S. Bäßler et al., Ternary, single-crystalline $\mathrm{Bi}_{2}(\mathrm{Te}, \mathrm{Se})_{3}$ nanowires grown by electrodeposition. Acta Mater. 125, 238-245 (2017). https:// doi.org/10.1016/j.actamat.2016.11.057 
148. L. Li, C. Jin, S. Xu, J. Yang, H. Du et al., Thermal conductivity of a single $\mathrm{Bi}_{0.5} \mathrm{Sb}_{1.5} \mathrm{Te}_{3}$ single-crystalline nanowire. Nanotechnology 25(41), 415704 (2014)

149. S. Bäßler, T. Böhnert, J. Gooth, C. Schumacher, E. Pippel et al., Thermoelectric power factor of ternary single-crystalline $\mathrm{Sb}_{2} \mathrm{Te}_{3}$-and $\mathrm{Bi}_{2} \mathrm{Te}_{3}$-based nanowires. Nanotechnology 24(49), 495402 (2013)

150. C.-G. Kuo, Y.-T. Hsieh, C.-F. Yang, C.-H. Huang, C.-Y. Yen, Growth of anodic aluminum oxide templates and the application in fabrication of the BiSbTe-based thermoelectric nanowires. Int. J. Photoenergy (2014). https://doi.org/10. 1155/2014/978184

151. M. Hasan, D. Gautam, R. Enright, Electrodeposition of textured $\mathrm{Bi}_{27} \mathrm{Sb}_{28} \mathrm{Te}_{45}$ nanowires with enhanced electrical conductivity. Mater. Chem. Phys. 173, 438-445 (2016). https:// doi.org/10.1016/j.matchemphys.2016.02.035

152. A. Danine, J. Schoenleber, J. Ghanbaja, F. Montaigne, C. Boulanger et al., Microstructure and thermoelectric properties of p-type bismuth antimony telluride nanowires synthetized by template electrodeposition in polycarbonate membranes. Electrochim. Acta 279, 258-268 (2018). https://doi. org/10.1016/j.electacta.2018.05.071

153. A. Datta, A. Sangle, N. Hardingham, C. Cooper, M. Kraan et al., Structure and thermoelectric properties of $\mathrm{Bi}_{2-\mathrm{x}} \mathrm{Sb}_{\mathrm{x}} \mathrm{Te}_{3}$ nanowires grown in flexible nanoporous polycarbonate templates. Materials 10(5), 553 (2017). https://doi.org/10.3390/ ma10050553

154. J. Fu, J. Shen, H. Shi, Y. Liang, Z. Qu et al., Preparation and characterisation of single-crystalline structure $\mathrm{Sb} / \mathrm{Bi}_{2} \mathrm{Te}_{3}$ superlattice nanowires. Micro Nano Lett. 11(11), 738-740 (2016). https://doi.org/10.1049/mnl.2015.0457

155. B. Yoo, F. Xiao, K.N. Bozhilov, J. Herman, M.A. Ryan et al., Electrodeposition of thermoelectric superlattice nanowires. Adv. Mater. 19(2), 296-299 (2007). https://doi.org/10.1002/ adma.200600606

156. S.J. Limmer, W.G. Yelton, M.P. Siegal, J.L. Lensch-Falk, J. Pillars et al., Electrochemical deposition of $\mathrm{Bi}_{2}(\mathrm{Te}, \mathrm{Se})_{3}$ nanowire arrays on Si. J. Electrochem. Soc. 159(4), D235 (2012)

157. X. Li, K. Cai, D. Yu, Y. Wang, Electrodeposition and characterization of thermoelectric $\mathrm{Bi}_{2} \mathrm{Te}_{2} \mathrm{Se} / \mathrm{Te}$ multilayer nanowire arrays. Superlattices Microstruct. 50(5), 557-562 (2011). https://doi.org/10.1016/j.spmi.2011.09.001

158. D. Li, J.T. McCann, Y. Xia, M. Marquez, Electrospinning: A simple and versatile technique for producing ceramic nanofibers and nanotubes. J. Am. Ceram. Soc. 89(6), 1861-1869 (2006). https://doi.org/10.1111/j.1551-2916.2006.00989.x

159. S.-X. Wang, C.C. Yap, J. He, C. Chen, S.Y. Wong et al., Electrospinning: A facile technique for fabricating functional nanofibers for environmental applications. Nanotechnol. Rev. 5(1), 51-73 (2016). https://doi.org/10.1515/ntrev-2015-0065

160. T. Yin, D. Liu, Y. Ou, F. Ma, S. Xie et al., Nanocrystalline thermoelectric $\mathrm{Ca}_{3} \mathrm{Co}_{4} \mathrm{O}_{9}$ ceramics by sol-gel based electrospinning and spark plasma sintering. J. Phys. Chem. C 114(21), 10061-10065 (2010). https://doi.org/10.1021/jp102 4872
161. F. Ma, Y. Ou, Y. Yang, Y. Liu, S. Xie et al., Nanocrystalline structure and thermoelectric properties of electrospun $\mathrm{NaCo}_{2} \mathrm{O}_{4}$ nanofibers. J. Phys. Chem. C 114(50), 2203822043 (2010). https://doi.org/10.1021/jp107488k

162. W. Xu, Y. Shi, H. Hadim, The fabrication of thermoelectric $\mathrm{La}_{0.95} \mathrm{Sr}_{0.05} \mathrm{CoO}_{3}$ nanofibers and Seebeck coefficient measurement. Nanotechnology 21(39), 395303 (2010)

163. K.-R. Park, H.-B. Cho, Y. Song, S. Kim, Y.-T. Kwon et al., Large-scale synthesis of lead telluride ( $\mathrm{PbTe}$ ) nanotube-based nanocomposites with tunable morphology, crystallinity and thermoelectric properties. Appl. Surf. Sci. 436, 785-790 (2018). https://doi.org/10.1016/j.apsusc.2017.12.102

164. M. Zhang, S.-D. Park, J. Kim, M. Nalbandian, S. Kim et al., Synthesis and thermoelectric characterization of lead telluride hollow nanofibers. Front. Chem. 6, 436 (2018). https:// doi.org/10.3389/fchem.2018.00436

165. K.S. Novoselov, A.K. Geim, S.V. Morozov, D. Jiang, Y. Zhang et al., Electric field effect in atomically thin carbon films. Science 306(5696), 666-669 (2004). https://doi.org/ 10.1126/science. 1102896

166. Q.H. Wang, K. Kalantar-Zadeh, A. Kis, J.N. Coleman, M.S. Strano, Electronics and optoelectronics of two-dimensional transition metal dichalcogenides. Nat. Nanotechnol. 7(11), 699-712 (2012). https://doi.org/10.1038/nnano.2012.193

167. X. Zhang, Y. Xie, Recent advances in free-standing twodimensional crystals with atomic thickness: design, assembly and transfer strategies. Chem. Soc. Rev. 42(21), 8187-8199 (2013). https://doi.org/10.1039/C3CS60138B

168. M. Xu, T. Liang, M. Shi, H. Chen, Graphene-like two-dimensional materials. Chem. Rev. 113(5), 3766-3798 (2013). https://doi.org/10.1021/cr300263a

169. H. Zhang, W. Zeng, C. Pan, L. Feng, M. Ou et al., SnTe@ $\mathrm{MnO}_{2}$-SP nanosheet-based intelligent nanoplatform for second near-infrared light-mediated cancer theranostics. Adv. Funct. Mater. 29(37), 1903791 (2019). https://doi.org/10. 1002/adfm.201903791

170. J.Y. Oh, J.H. Lee, S.W. Han, S.S. Chae, E.J. Bae et al., Chemically exfoliated transition metal dichalcogenide nanosheetbased wearable thermoelectric generators. Energy Environ. Sci. 9(5), 1696-1705 (2016). https://doi.org/10.1039/C5EE0 $3813 \mathrm{H}$

171. Y. Huang, L. Li, Y.-H. Lin, C.-W. Nan, Liquid exfoliation few-layer snse nanosheets with tunable band gap. J. Phys. Chem. C 121(32), 17530-17537 (2017). https://doi.org/10. 1021/acs.jpcc.7b06096

172. W.J. Baumgardner, J.J. Choi, Y.-F. Lim, T. Hanrath, Snse nanocrystals: synthesis, structure, optical properties, and surface chemistry. J. Am. Chem. Soc. 132(28), 9519-9521 (2010). https://doi.org/10.1021/ja1013745

173. Y.X. Chen, Z.H. Ge, M. Yin, D. Feng, X.Q. Huang et al., Understanding of the extremely low thermal conductivity in high-performance polycrystalline snse through potassium doping. Adv. Funct. Mater. 26(37), 6836-6845 (2016). https://doi.org/10.1002/adfm.201602652

174. J.-H. Ahn, M.-J. Lee, H. Heo, J.H. Sung, K. Kim et al., Deterministic two-dimensional polymorphism growth of hexagonal 
n-type $\mathrm{SnS}_{2}$ and orthorhombic p-type $\mathrm{SnS}$ crystals. Nano Lett. 15(6), 3703-3708 (2015). https://doi.org/10.1021/acs. nanolett.5b00079

175. X.-H. Ma, K.-H. Cho, Y.-M. Sung, Growth mechanism of vertically aligned $\mathrm{SnSe}$ nanosheets via physical vapour deposition. CrystEngComm 16(23), 5080-5086 (2014). https:// doi.org/10.1039/C4CE00213J

176. Z. Tian, C. Guo, M. Zhao, R. Li, J. Xue, Two-dimensional sns: A phosphorene analogue with strong in-plane electronic anisotropy. ACS Nano 11(2), 2219-2226 (2017). https://doi. org/10.1021/acsnano.6b08704

177. S. Liu, N. Sun, M. Liu, S. Sucharitakul, X.P. Gao, Nanostructured snse: Synthesis, doping, and thermoelectric properties. J. Appl. Phys. 123(11), 115109 (2018). https://doi.org/10. $1063 / 1.5018860$

178. H.-A. Chen, H. Sun, C.-R. Wu, Y.-X. Wang, P.-H. Lee et al., Single-crystal antimonene films prepared by molecular beam epitaxy: Selective growth and contact resistance reduction of the 2D material heterostructure. ACS Appl. Mater. Interfaces 10(17), 15058-15064 (2018). https://doi.org/10.1021/acsami. $8 \mathrm{~b} 02394$

179. M. Fortin-Deschênes, O. Waller, T. Mentes, A. Locatelli, S. Mukherjee et al., Synthesis of antimonene on germanium. Nano Lett. 17(8), 4970-4975 (2017). https://doi.org/10.1021/ acs.nanolett.7b02111

180. X. Wu, Y. Shao, H. Liu, Z. Feng, Y.L. Wang et al., Epitaxial growth and air-stability of monolayer antimonene on $\mathrm{PdTe}_{2}$. Adv. Mater. 29(11), 1605407 (2017). https://doi.org/10.1002/ adma.201605407

181. T. Nagao, T. Doi, T. Sekiguchi, S. Hasegawa, Epitaxial growth of single-crystal ultrathin films of bismuth on $\mathrm{Si}$ (111). Jpn. J. Appl. Phys. 39(7S), 4567 (2000)

182. T. Nagao, J. Sadowski, M. Saito, S. Yaginuma, Y. Fujikawa et al., Nanofilm allotrope and phase transformation of ultrathin bi film on Si (111)-7×7. Phys. Rev. Lett. 93(10), 105501 (2004). https://doi.org/10.1103/PhysRevLett.93. 105501

183. T. Aizawa, I. Ohkubo, M.S. Lima, T. Sakurai, T. Mori, Fabrication of $\mathrm{Mg}_{2} \mathrm{Sn}$ (111) film by molecular beam epitaxy. $\mathrm{J}$. Vac. Sci. Technol. A: Vac. Surfaces Films 37(6), 061513 (2019). https://doi.org/10.1116/1.5122844

184. Z. Ye, S. Cui, T. Shu, S. Ma, Y. Liu et al., Electronic band structure of epitaxial pbte (111) thin films observed by angleresolved photoemission spectroscopy. Phys. Rev. B 95(16), 165203 (2017). https://doi.org/10.1103/PhysRevB.95.165203

185. W. Ren, H. Li, L. Gao, Y. Li, Z. Zhang et al., Epitaxial growth and thermal-conductivity limit of single-crystalline $\mathrm{Bi}_{2} \mathrm{Se}_{3} /$ $\mathrm{In}_{2} \mathrm{Se}_{3}$ superlattices on mica. Nano Res. 10(1), 247-254 (2017). https://doi.org/10.1007/s12274-016-1282-8

186. M.-W. Chen, H. Kim, D. Ovchinnikov, A. Kuc, T. Heine et al., Large-grain mbe-grown gase on gaas with a mexican hatlike valence band dispersion. NPJ 2D Mater. Appl. 2(1), 1-7 (2018). https://doi.org/10.1038/s41699-017-0047-x

187. A. Rice, J. Kawasaki, N. Verma, D. Pennachio, B. Schultz et al., Structural and electronic properties of molecular beam epitaxially grown $\mathrm{Ni}_{1+x}$ TiSn films. J. Crystal Growth 467, 71-76 (2017). https://doi.org/10.1016/j.jcrysgro.2017.03.015

188. X. Chen, R. Fan, Low-temperature hydrothermal synthesis of transition metal dichalcogenides. Chem. Mater. 13(3), 802-805 (2001). https://doi.org/10.1021/cm000517+

189. X. Zhang, G. Ma, J. Wang, Hydrothermal synthesis of twodimensional $\mathrm{MoS}_{2}$ and its applications. Tungsten 1(1), 59-79 (2019). https://doi.org/10.1007/s42864-019-00014-9

190. U. Alli, S.J. Hettiarachchi, S. Kellici, Chemical functionalisation of 2D materials by batch and continuous hydrothermal flow synthesis. Chem. Eur. J. 26(29), 6447-6460 (2020). https://doi.org/10.1002/chem.202000383

191. X.L. Shi, X. Tao, J. Zou, Z.G. Chen, High-performance thermoelectric SnSe: aqueous synthesis, innovations, and challenges. Adv. Sci. 7(7), 1902923 (2020). https://doi.org/10. 1002/advs.201902923

192. S. Chandra, K. Biswas, Realization of high thermoelectric figure of merit in solution synthesized 2D SnSe nanoplates via Ge alloying. J. Am. Chem. Soc. 141(15), 6141-6145 (2019). https://doi.org/10.1021/jacs.9b01396

193. W. Shi, L. Zhou, S. Song, J. Yang, H. Zhang, Hydrothermal synthesis and thermoelectric transport properties of impurityfree antimony telluride hexagonal nanoplates. Adv. Mater. 20(10), 1892-1897 (2008). https://doi.org/10.1002/adma. 200702003

194. J.G. Park, Y.H. Lee, High thermoelectric performance of Bi-Te alloy: defect engineering strategy. Curr. Appl. Phys. 16(9), 1202-1215 (2016). https://doi.org/10.1016/j.cap.2016. 03.028

195. Y. Hosokawa, K. Tomita, M. Takashiri, Growth of singlecrystalline $\mathrm{Bi}_{2} \mathrm{Te}_{3}$ hexagonal nanoplates with and without single nanopores during temperature-controlled solvothermal synthesis. Sci. Rep. 9(1), 10790 (2019). https://doi.org/10. 1038/s41598-019-47356-5

196. R. Mori, Y. Mayuzumi, M. Yamaguchi, A. Kobayashi, Y. Seki et al., Improved thermoelectric properties of solvothermally synthesized $\mathrm{Bi}_{2} \mathrm{Te}_{3}$ nanoplate films with homogeneous interconnections using $\mathrm{Bi}_{2} \mathrm{Te}_{3}$ electrodeposited layers. J. Alloys Compd. 818, 152901 (2020). https://doi.org/10.1016/j.jallc om.2019.152901

197. Y. Wang, W.-D. Liu, H. Gao, L.-J. Wang, M. Li et al., High porosity in nanostructured n-type $\mathrm{Bi}_{2} \mathrm{Te}_{3}$ obtaining ultralow lattice thermal conductivity. ACS Appl. Mater. Interfaces 11(34), 31237-31244 (2019). https://doi.org/10.1021/acsami. 9b12079

198. M. Hong, T.C. Chasapis, Z.-G. Chen, L. Yang, M.G. Kanatzidis et al., $\mathrm{N}$-type $\mathrm{Bi}_{2} \mathrm{Te}_{3-\mathrm{x}} \mathrm{Se}_{\mathrm{x}}$ nanoplates with enhanced thermoelectric efficiency driven by wide-frequency phonon scatterings and synergistic carrier scatterings. ACS Nano 10(4), 4719-4727 (2016). https://doi.org/10.1021/acsnano.6b01156

199. Y. Zhang, Y. Liu, C. Xing, T. Zhang, M. Li et al., Tin selenide molecular precursor for the solution processing of thermoelectric materials and devices. ACS Appl. Mater. Interfaces 12(24), 27104-27111 (2020). https://doi.org/10.1021/acsami. 0c04331 
200. S. Saha, A. Banik, K. Biswas, Few-layer nanosheets of n-type $\mathrm{SnSe}_{2}$. Chem. Eur. J. 22(44), 15634-15638 (2016). https:// doi.org/10.1002/chem.201604161

201. S. Chandra, A. Banik, K. Biswas, N-type ultrathin few-layer nanosheets of bi-doped SnSe: synthesis and thermoelectric properties. ACS Energy Lett. 3(5), 1153-1158 (2018). https:// doi.org/10.1021/acsenergylett.8b00399

202. N.A. Rongione, M. Li, H. Wu, H.D. Nguyen, J.S. Kang et al., High-performance solution-processable flexible $\mathrm{SnSe}$ nanosheet films for lower grade waste heat recovery. Adv. Electron. Mater. 5(3), 1800774 (2019). https://doi.org/10. 1002/aelm.201800774

203. M. Hong, Z.-G. Chen, L. Yang, T.C. Chasapis, S.D. Kang et al., Enhancing the thermoelectric performance of $\mathrm{SnSe}_{1-\mathrm{x}} \mathrm{Te}_{\mathrm{x}}$ nanoplates through band engineering. J. Mater. Chem. A 5(21), 10713-10721 (2017). https://doi.org/10. 1039/C7TA02677C

204. G. Han, S.R. Popuri, H.F. Greer, J.W.G. Bos, W. Zhou et al., Facile surfactant-free synthesis of p-type SnSe nanoplates with exceptional thermoelectric power factors. Angew. Chem. Int. Ed. 55(22), 6433-6437 (2016). https://doi.org/10.1002/ anie. 201601420

205. J.S. Son, M.K. Choi, M.-K. Han, K. Park, J.-Y. Kim et al., $\mathrm{N}$-type nanostructured thermoelectric materials prepared from chemically synthesized ultrathin $\mathrm{Bi}_{2} \mathrm{Te}_{3}$ nanoplates. Nano Lett. 12(2), 640-647 (2012). https://doi.org/10.1021/ nl203389x

206. M. Martín-González, O. Caballero-Calero, P. Díaz-Chao, Nanoengineering thermoelectrics for $21^{\text {st }}$ century: Energy harvesting and other trends in the field. Renew. Sust. Energ. Rev. 24, 288-305 (2013). https://doi.org/10.1016/j.rser.2013. 03.008

207. B. Xu, T. Feng, Z. Li, S.T. Pantelides, Y. Wu, Constructing highly porous thermoelectric monoliths with high-performance and improved portability from solution-synthesized shape-controlled nanocrystals. Nano Lett. 18(6), 4034-4039 (2018). https://doi.org/10.1021/acs.nanolett.8b01691

208. J. Shi, H. Chen, S. Jia, W. Wang, 3d printing fabrication of porous bismuth antimony telluride and study of the thermoelectric properties. J. Manuf. Process 37, 370-375 (2019). https://doi.org/10.1016/j.jmapro.2018.11.001

209. S. Hong, J. Park, S.G. Jeon, K. Kim, S.H. Park et al., Monolithic $\mathrm{Bi}_{1.5} \mathrm{Sb}_{0.5} \mathrm{Te}_{3}$ ternary alloys with a periodic $3 \mathrm{D}$ nanostructure for enhancing thermoelectric performance. J. Mater. Chem. C 5(35), 8974-8980 (2017). https://doi.org/10.1039/ C7TC02717F

210. K. Kim, J. Park, S. Hong, S.H. Park, S.G. Jeon et al., Anomalous thermoelectricity of pure $\mathrm{ZnO}$ from $3 \mathrm{D}$ continuous ultrathin nanoshell structures. Nanoscale 10(6), 3046-3052 (2018). https://doi.org/10.1039/C7NR08167G

211. G. Tang, W. Wei, J. Zhang, Y. Li, X. Wang et al., Realizing high figure of merit in phase-separated polycrystalline $\mathrm{Sn}_{1-\mathrm{x}} \mathrm{Pb}_{\mathrm{x}} \mathrm{Se}$. J. Am. Chem. Soc. 138(41), 13647-13654 (2016). https://doi.org/10.1021/jacs.6b07010

212. G. Tang, J. Liu, J. Zhang, D. Li, K.H. Rara et al., Realizing high thermoelectric performance below phase transition temperature in polycrystalline snse via lattice anharmonicity strengthening and strain engineering. ACS Appl. Mater. Interfaces 10(36), 30558-30565 (2018). https://doi.org/10. 1021/acsami.8b10056

213. Y. Gong, C. Chang, W. Wei, J. Liu, W. Xiong et al., Extremely low thermal conductivity and enhanced thermoelectric performance of polycrystalline $\mathrm{SnSe}$ by $\mathrm{Cu}$ doping. Scripta Mater. 147, 74-78 (2018). https://doi.org/10.1016/j.scrip tamat.2017.12.035

214. R. Xu, L. Huang, J. Zhang, D. Li, J. Liu et al., Nanostructured snse integrated with se quantum dots with ultrahigh power factor and thermoelectric performance from magnetic fieldassisted hydrothermal synthesis. J. Mater. Chem. A 7(26), 15757-15765 (2019). https://doi.org/10.1039/C9TA03967H

215. S. Li, X. Lou, X. Li, J. Zhang, D. Li et al., Realization of high thermoelectric performance in polycrystalline tin selenide through schottky vacancies and endotaxial nanostructuring. Chem. Mater. 32(22), 9761-9770 (2020). https://doi.org/10. 1021/acs.chemmater.0c03657

216. X. Shi, Z.-G. Chen, W. Liu, L. Yang, M. Hong et al., Achieving high figure of merit in p-type polycrystalline $\mathrm{Sn}_{0.98} \mathrm{Se}$ via self-doping and anisotropy-strengthening. Energy Storage Mater. 10, 130-138 (2018). https://doi.org/10.1016/j.ensm. 2017.08.014

217. X. Shi, A. Wu, W. Liu, R. Moshwan, Y. Wang et al., Polycrystalline snse with extraordinary thermoelectric property via nanoporous design. ACS Nano 12(11), 11417-11425 (2018). https://doi.org/10.1021/acsnano.8b06387

218. X. Shi, K. Zheng, M. Hong, W. Liu, R. Moshwan et al., Boosting the thermoelectric performance of p-type heavily $\mathrm{Cu}$-doped polycrystalline $\mathrm{SnSe}$ via inducing intensive crystal imperfections and defect phonon scattering. Chem. Sci. 9(37), 7376-7389 (2018). https://doi.org/10.1039/C8SC0 2397B

219. X.L. Shi, W.D. Liu, A.Y. Wu, V.T. Nguyen, H. Gao et al., Optimization of sodium hydroxide for securing high thermoelectric performance in polycrystalline $\mathrm{Sn}_{1-\mathrm{x}} \mathrm{Se}$ via anisotropy and vacancy synergy. InfoMat 2(6), 1201-1215 (2019). https://doi.org/10.1002/inf2.12057

220. Y. Zheng, X.-L. Shi, H. Yuan, S. Lu, X. Qu et al., A synergy of strain loading and laser radiation in determining the high-performing electrical transports in the single $\mathrm{Cu}$-doped SnSe microbelt. Mater. Today Phys. (2020). https://doi.org/ 10.1016/j.mtphys.2020.100198

221. Y. Liu, G. García, S. Ortega, D. Cadavid, P. Palacios et al., Solution-based synthesis and processing of $\mathrm{Sn}$-and $\mathrm{Bi}$-doped $\mathrm{Cu}_{3} \mathrm{SbSe}_{4}$ nanocrystals, nanomaterials and ring-shaped thermoelectric generators. J. Mater. Chem. A 5(6), 2592-2602 (2017). https://doi.org/10.1039/C6TA08467B

222. L. De Trizio, L. Manna, Forging colloidal nanostructures via cation exchange reactions. Chem. Rev. 116(18), 1085210887 (2016). https://doi.org/10.1021/acs.chemrev.5b00739

223. F. Yang, J. Wu, A. Suwardi, Y. Zhao, B. Liang et al., Gatetunable polar optical phonon to piezoelectric scattering in few-layer Bi2O2Se for high-performance thermoelectrics. Adv. Mater. (2020). https://doi.org/10.1002/adma.202004786 
224. S. Cecchi, D. Dragoni, D. Kriegner, E. Tisbi, E. Zallo et al., Interplay between structural and thermoelectric properties in epitaxial $\mathrm{Sb}_{2+\mathrm{x}} \mathrm{Te}_{3}$ alloys. Adv. Funct. Mater. 29(2), 1805184 (2019). https://doi.org/10.1002/adfm.201805184

225. D. Bao, J. Chen, Y. Yu, W. Liu, L. Huang et al., Texturedependent thermoelectric properties of nano-structured $\mathrm{Bi}_{2} \mathrm{Te}_{3}$. Chem. Eng. J. 388, 124295 (2020). https://doi.org/ 10.1016/j.cej.2020.124295

226. Y. Zhang, Y. Liu, C. Xing, T. Zhang, M. Li et al., Tin selenide molecular precursor for the solution-processing of thermoelectric materials and devices. ACS Appl. Mater. Interfaces 12(24), 27104-27111 (2020). https://doi.org/10. 1021/acsami.0c04331

227. X. Shi, A. Wu, T. Feng, K. Zheng, W. Liu et al., High thermoelectric performance in p-type polycrystalline Cd-doped SnSe achieved by a combination of cation vacancies and localized lattice engineering. Adv. Energy Mater. 9(11), 1803242 (2019). https://doi.org/10.1002/aenm.201803242 